\title{
Extracellular Vesicles from Human Adipose-Derived Mesenchymal Stem Cells: A Review of Common Cargos
}

\author{
Maria Luz Alonso-Alonso ${ }^{1}$ (D) - Laura García-Posadas ${ }^{1}$ (D) Yolanda Diebold $^{1,2}$ (D)
}

Accepted: 14 March 2021 / Published online: 26 April 2021

(C) The Author(s) 2021

\begin{abstract}
In recent years, the interest in adipose tissue mesenchymal cell-derived extracellular vesicles (AT-MSC-EVs) has increasingly grown. Numerous articles support the potential of human AT-MSC-EVs as a new therapeutic option for treatment of diverse diseases in the musculoskeletal and cardiovascular systems, kidney, skin, and immune system, among others. This approach makes use of the molecules transported inside of EVs, which play an important role in cell communication and in transmission of macromolecules. However, to our knowledge, there is no database where essential information about AT-MSC-EVs cargo molecules is gathered for easy reference. The aim of this study is to describe the different molecules reported so far in ATMSC- EVs, their main molecular functions, and biological processes in which they are involved. Recently, the presence of 591 proteins and 604 microRNAs (miRNAs) has been described in human AT-MSC-EVs. The main molecular function enabled by both proteins and miRNAs present in human AT-MSC-EVs is the binding function. Signal transduction and gene silencing are the biological processes in which a greater number of proteins and miRNAs from human AT-MSC-EVs are involved, respectively. In this review we highlight the therapeutics effects of AT-MSC-EVs related with their participation in relevant biological processes including inflammation, angiogenesis, cell proliferation, apoptosis and migration, among others.
\end{abstract}

Keywords Extracellular vesicles $\cdot$ Adipose-derived mesenchymal stem cells $\cdot$ miRNA $\cdot$ Proteomic $\cdot$ Exosome

\section{Introduction}

"Extracellular vesicle" (EV) is defined by the International Society for Extracellular Vesicles (ISEV) as the "generic term for particles naturally released from the cell that are delimited by a lipid bilayer and cannot replicate, i.e. do not contain a functional nucleus" $[1,2]$. These particles contain a significant variety of proteins and RNAs that play important roles in cellcell communication and in transmission of macromolecules between cells [3-6]. As this feature makes EVs a potential therapeutic approach for various diseases, interest in EV research has significantly increased over the last decade $[4,7]$. Importantly, the profile of EV cargo depends on the cell type

Maria Luz Alonso-Alonso

marialuz.alonso.alonso@gmail.com

1 Ocular Surface Group, Instituto de Oftalmobiología Aplicada (IOBA), Universidad de Valladolid, Valladolid, Spain

2 Centro de Investigación Biomédica en Red en el área temática de Bioingeniería, Biomateriales y Nanomedicina (CIBER-BBN), Valladolid, Spain of origin [8]. In this sense, although a wide range of mammalian cells release EVs [4, 9], mesenchymal stem cells (MSC) are considered one of the most prolific producer cell types [10]. These vesicles are involved in the paracrine properties of MSCs [11-13].

MSCs can be harvested from different tissues, such as bone marrow (BM), adipose tissue (AT), dental pulp, and umbilical cord, among others $[14,15]$. BM and AT are the most common sources of MSC for use in research [16-19]. Although BM-MSCs were the first identified MSC [20] type and have been extensively studied [21], AT-MSCs present remarkable advantages by comparison, including higher stability in culture conditions and lower senescence ratio [21]. In addition, the amount of MSC that can be obtained from this tissue, which is usually treated as waste material and discarded [22, 23], is significantly greater than that obtained from BM aspirates [21].

The interest in AT-MSC-EVs has increasingly grown, due to the wide range of AT sources and their relatively easy accessibility [9]. AT-MSC-EVs have been isolated not only from human cells, but also from mouse [24-32], rat [33, 34], pig [35-38], and rabbit [39, 40] cells. The main objective of 
most published studies on AT-MSC-EVs was to evaluate their potential use as a new therapeutic approach to treat various diseases. Moreover, several of these publications did include an analysis of the molecules transported by the EVs, which is especially relevant to understanding their mechanism of action beyond their observable effects. Taken together, these studies have confirmed the presence of 591 proteins and 604 microRNA (miRNA) in the AT-MSC-EVs. Nevertheless, evaluation of effects of the molecules identified in the cargo focused solely on the disease or tissues under study. However, independent of the specific therapeutic use, the human ATMSC-EVs are compositionally identical. Therefore, we anticipate that a review collecting together all available information about AT-MSC-EVs cargo and their function will be extremely useful for researchers working in this field.

ISEV recently published a guideline encouraging researchers to report their data to these field-specific databases to detect different studies describing the same molecules [1]. Thus, there is a great need for a well-organised review that collects all relevant information regarding molecules identified so far in AT-MSC-EVs cargo, and their biological activities. This will facilitate future research in this area. Currently, there are two online databases collecting the identified molecules in cargos of EVs derived from different cell types: http:// microvesicles.org [41] (formerly http://www.exocarta.org [42]), and http://evpedia.info [43] (link currently unavailable). Both databases are good, reliable sources of information; however, the information available on ATMSC-EVs cargo is still limited compared to that available on other cell types, such as T cells or prostate cancer cell EV cargos. Thus, this review will provide an updated source not only of identified AT-MSC-EVs cargo molecules, but also their functions and potential therapeutic applications.

Given the growing interest in the MSC-EVs, especially in those derived from AT, the purpose of this study is to provide the AT-MSC research community with a systematic review of publications reporting the cargo of AT-MSC-EVs, including an analysis of their molecular functions and the biological process in which they are involved.

\section{Methods}

A systematic literature search was conducted in the medical databases Pubmed and Web of Science, using the keywords "extracellular vesicles", "exosome", "adipose mesenchymal stem cells", "cargo", "protein" and "miRNA" without setting a time limit (last searched 6th September 2020). 112 articles published between 2006 and 2020 (inclusive) were reviewed. 48 of these articles were related to human AT-MSC-EV, and 17 to AT-MSC-EVs in other species. The remaining articles were about EVs in general and MSC-EVs from other sources. This study has included both articles that used the nomenclature recommended by ISEV ("EV") [1] and those which used the terms "exosomes" and "microvesicles". Given the number of publications that have used these terms during the past decades [2], we considered that the exclusion of them could lead to the loss of relevant information. In addition, although the isolation methods of EVs could have an impact on the cargo composition, it was not an exclusion criterion since there is no single optimal separation method [1].

Different nomenclatures such as adipose stem cells, adipose stromal cells, or adipose-derived stem cells, have been used to identify AT-MSCs. The keyword "adipose mesenchymal stem cells" allowed us to find articles in which authors used several of these nomenclatures. However, we may have missed some information due to this great variety of terms, and this may be a limitation of the present study.

Information regarding proteins (10 articles) and RNA (16 articles) detected in human AT-MSC-EVs was collected in two databases created in Excel (Microsoft Office Excel 2013; Microsoft Corporation, Redmond, WA, USA). Although an article was found in which the lipid content of human AT-MSC-ECs was measured, no more information about lipids was reported. Therefore, it was not possible to include a database of lipids in this review.

To standardise the data and facilitate the recognition of identified proteins, we used the recommended name and identifier code proposed by the Universal Protein Knowledgebase [44] (UniProtKB). This database includes additional information about the short and alternative names for some proteins, which allowed us to identify proteins described by certain authors with these terms. UniProtKB host institutions are the European Bioinformatics Institute (EMBL-EBI), the Swiss Institute of Bioinformatics, and the Protein Information Resource.

For RNA, we used the name of mature micro RNAs (miRNAs) and the code of identification recommended by the RNAcentral database [45] (https://rnacentral.org/). This database is coordinated by EMBL-EBI and integrates information from 41 Expert Databases out of the 53 which constitute the RNAcentral Consortium. In addition, we used the miRBase database [46-51] to classify miRNAs by gene families. miRBase is one of the Expert Databases integrated in the RNAcentral database, and is managed by the University of Manchester. This database also includes information about the previous nomenclature of some miRNAs, which allowed us to correlate the previous miRNA name used by certain authors with the current recommended terminology.

Messenger RNA (mRNA) [52], transfer RNA (tRNA), small ribosomal RNA (rRNA), small nuclear RNA (snRNA), small nucleolar RNA (snoRNA) and small cytoplasmic RNA (scRNA) are also present in AT-MSC-EVs $[53,54]$. However, there is less information available on these, therefore, it was possible to include the list of the main tRNAs and mRNA present in AT-MSC-EVs, but not the other types of RNA. 
Finally, the web-based tool QuickGO [55] (https://www. ebi.ac.uk/QuickGO/), also managed by EMBL-EBI, was used to search the gene ontology $(\mathrm{GO})$ terms of molecular functions and biological processes of detected proteins and miRNAs. An ontology consists of a set of specific concepts with welldefined relationships between them. The GO was developed by the GO Consortium, as a tool to unify the terminology used to describe the functions of genes and gene products [56].

\section{Cargo of AT-MSC-EVs}

Human AT-MSC-EVs transport different types of proteins [12, 52, 57-65], RNAs [11, 12, 53, 54, 59, 64-74] and lipids [58]. Due to this variety of cargo molecules, AT-MSC-EVs are involved in a wide range of biological functions including migration, immune regulation, cell proliferation, angiogenesis, osteocyte metabolism and nerve regeneration (for a comprehensive review see ref. 9) [9]. Their therapeutic potential is being tested for the treatment of diverse diseases in musculoskeletal [12, 52, 57, 65-67, 75-78] and cardiovascular systems [60, 72, 79-81], nephrology [82, 83], skin [62, 68, 84-86] and immunology [71, 87], among others.

Surprisingly, we could only find one published study about the potential of human AT-MSC-EVs for the treatment of eye diseases [88], despite the fact that human AT-MSC and their conditioned media are being used in ophthalmology [89-99]. For instance, they are being used in 6 out of 403 registered clinical trials with these cells (ClinicalTrials.gov, NCT04484402 NCT03878628, NCT02932852, NCT01808378, NCT02144103 and NCT02024269). In this study, human AT-MSC-EVs showed a protective effect both in vitro and in vivo in a mouse model of dry eye by suppressing the NLRP3 (NOD-like receptor family) inflammasome activation [88]. Moreover, the positive effects of mouse and rabbit AT-MSC-EVs have been demonstrated in in vivo models of laser-induced retinal injury [29] and diabetic retinopathy [40], respectively. In addition, rabbit AT-MSC-EVs seemed to take part in the viability regulation of cultured rabbit corneal stromal cells [39]. There are also several studies which have used human BM-MSC-EVs in ophthalmology, showing their beneficial effects in rat retinal and retinal ganglion cell cultures $[100,101]$ and in animal models of glaucoma $[102,103]$ and optic nerve crush [101]. As well as ATMSC, BM-MSC have also been widely used in ophthalmology [104-113], including 8 out of 293 registered clinical trials with these cells (ClinicalTrials.gov, NCT01531348, NCT01562002 [114], NCT01920867 [115, 116], NCT02325843, NCT02330978, NCT03011541 [117], NCT03173638 and NCT03967275).

In the present review, we comprehensively describe the $\mathrm{GO}$ annotations of molecular functions and biological processes of each type of cargo reported in human ATMSC-EVs.

\section{Proteins}

Proteomic analysis of EV cargo can enhance the knowledge of the functions and mechanisms of action in which these vesicles are involved [28]. To analyse AT-MSC-EVs protein content, researchers used a large variety of techniques such as mass spectrometry $[12,57,59]$, antibody arrays $[52,60,61$, 65], Western Blotting [62, 63] and, to a lesser extent, rate immune nephelometry [58]. The EVs in those studies have been isolated by ultracentrifugation [12, 52, 57, 60, 65], filtration and ultracentrifugation $[61,63]$, commercial EV isolation kits [62], ultrafiltration [58], and affinity purification [59].

So far, 591 proteins have been identified (Table 1). Nevertheless, taking into account both the name and the gene or NCBI Reference Sequences mentioned in the articles, it was not possible to connect the proteins C-peptide, HCR/ CRAM-A/B [52, 65], INSL3, macroglobulin [65], CA 19-9, MSHa, PPARg2, TGF-beta 5 and TRA-1-60/TRA-1-81, Pepsinogen I [52] with an UniprotKB code conclusively (Table 1). The presence of the protein families annexin, HSP 70 and HSP 90 has also been described [12] (Table 1). However, as the specific members of these three families were not reported, it was not possible to include them in the GO analyses.

The detailed molecular functions enabled by each protein are collected in Table 1S. The results showed that 577 proteins contribute to different molecular functions described by 710 GO terms. For the BMP-binding endothelial regulator protein, carcinoembryonic antigen-related cell adhesion molecule, coagulation factor XIII B chain and kremen protein 2, no GO annotations were found.

The main molecular functions enabled by the AT-MSCEVs proteins are described by specific child terms (more specific terms) of binding: protein binding ( $80 \%)$, metal ion binding (20\%), cytokine activity (18\%), identical protein binding (17\%), and signaling receptor binding (15\%) (Fig. 1). Therefore, binding seems to be the most relevant molecular function of AT-MSC-EVs. The number of AT-MSC-EVs proteins involved in each molecular function is variable. Most described molecular functions are enabled by a limited number of proteins (less than 10), and only $11.6 \%$ of the functions are enabled by 10 or more proteins. They are related by specific terms of four molecular functions: binding, catalytic activity, structural molecule activity and molecular transducer activity (Fig. 2).

578 of the AT-MSC-EVs proteins identified play a role in different biological processes described by 3884 GO terms. For carcinoembryonic antigen-related cell adhesion molecule 7, layilin, and sex hormone-binding globulin, no GO annotations were found. The proteins involved in each process are 
Table 1 Proteins detected in human AT-MSC-EVs in alphabetical order

\begin{tabular}{|c|c|c|c|c|}
\hline Protein & Abbreviation & UniProtKB & Gene & Ref. \\
\hline 5'-AMP-activated protein kinase catalytic subunit alpha- $1 *$ & AAPK1_HUMAN & Q13131 & PRKAA1 & {$[65]$} \\
\hline 72 kDa type IV collagenase* & MMP2_HUMAN & P08253 & MMP2 & {$[52]$} \\
\hline $\begin{array}{l}\text { A disintegrin and metalloproteinase with thrombospondin } \\
\text { motifs } 1 *\end{array}$ & ATS1_HUMAN & Q9UHI8 & ADAMTS1 & {$[65]$} \\
\hline $\begin{array}{l}\text { A disintegrin and metalloproteinase with thrombospondin } \\
\text { motifs } 2 *\end{array}$ & ATS2_HUMAN & O95450 & ADAMTS2 & {$[65]$} \\
\hline $\begin{array}{l}\text { A disintegrin and metalloproteinase with thrombospondin } \\
\text { motifs } 4^{*}\end{array}$ & ATS4_HUMAN & $\mathrm{O} 75173$ & ADAMTS4 & {$[52,65]$} \\
\hline $\begin{array}{l}\text { A disintegrin and metalloproteinase with thrombospondin } \\
\text { motifs } 17^{*}\end{array}$ & ATS17_HUMAN & Q8TE56 & ADAMTS17 & {$[52]$} \\
\hline $\begin{array}{l}\text { A disintegrin and metalloproteinase with thrombospondin } \\
\text { motifs } 18^{*}\end{array}$ & ATS18_HUMAN & Q8TE60 & ADAMTS18 & {$[65]$} \\
\hline $\begin{array}{l}\text { A disintegrin and metalloproteinase with thrombospondin } \\
\text { motifs } 19^{*}\end{array}$ & ATS19_HUMAN & Q8TE59 & ADAMTS19 & {$[52,65]$} \\
\hline Acidic fibroblast growth factor intracellular-binding protein & FIBP_HUMAN & O43427 & FIBP & {$[57]$} \\
\hline Activated CDC42 kinase $1 *$ & ACK1_HUMAN & Q07912 & TNK2 & {$[52,65]$} \\
\hline Activin receptor type-1B* & ACV1B_HUMAN & P36896 & ACVR1B & {$[65]$} \\
\hline Activin receptor type-2B* & AVR2B_HUMAN & Q13705 & ACVR2B & {$[65]$} \\
\hline Adenomatous polyposis coli protein* & APC_HUMAN & P25054 & APC & {$[52,65]$} \\
\hline Adhesion $\mathrm{G}$ protein-coupled receptor $\mathrm{B} 1 *$ & AGRB1_HUMAN & O14514 & ADGRB1 & {$[52]$} \\
\hline Adhesion G protein-coupled receptor E5* & AGRE5_HUMAN & P48960 & ADGRE5 & {$[52]$} \\
\hline ADP-ribosyl cyclase/cyclic ADP-ribose hydrolase $1 *$ & CD38_HUMAN & P28907 & $\mathrm{CD} 38$ & {$[65]$} \\
\hline Agouti-related protein* & AGRP_HUMAN & O00253 & AGRP & {$[52,65]$} \\
\hline Alkaline phosphatase, placental type* & PPB1_HUMAN & P05187 & ALPP & {$[52]$} \\
\hline Alpha-1-acid glycoprotein $1 *$ & A1AG1_HUMAN & P02763 & ORM1 & {$[65]$} \\
\hline Alpha-1-antitrypsin & A1AT_HUMAN & P01009 & SERPINA1 & {$[58]$} \\
\hline Alpha-1B-glycoprotein* & A1BG_HUMAN & P04217 & A1BG & {$[52,65]$} \\
\hline Alpha-fetoprotein* & FETA_HUMAN & P02771 & AFP & {$[52,59]$} \\
\hline Alpha-lactalbumin & LALBA_HUMAN & P00709 & LALBA & {$[52,65]$} \\
\hline Aminopeptidase $\mathrm{N}^{*}$ & AMPN_HUMAN & P15144 & ANPEP & {$[65]$} \\
\hline Amphiregulin & AREG_HUMAN & P15514 & AREG & {$[60,65]$} \\
\hline Angiopoietin-1 & ANGP1_HUMAN & Q15389 & ANGPT1 & {$[52,61,65]$} \\
\hline Angiopoietin-1 receptor* & TIE2_HUMAN & Q02763 & TEK & {$[61]$} \\
\hline Angiopoietin-4 & ANGP4_HUMAN & Q9Y264 & ANGPT4 & {$[65]$} \\
\hline Angiopoietin-related protein $1^{*}$ & ANGL1_HUMAN & O95841 & ANGPTL1 & {$[52]$} \\
\hline Angiopoietin-related protein $2 *$ & ANGL2_HUMAN & Q9UKU9 & ANGPTL2 & {$[65]$} \\
\hline Angiopoietin-related protein $7 *$ & ANGL7_HUMAN & O43827 & ANGPTL7 & {$[60]$} \\
\hline Angiostatin (cleaved from plasminogen) & PLMN_HUMAN & P00747 & PLG & {$[52,60,61]$} \\
\hline Annexin** & - & - & - & {$[12]$} \\
\hline Annexin A5 & ANXA5_HUMAN & P08758 & ANXA5 & {$[59]$} \\
\hline Annexin A7 & ANXA7_HUMAN & P20073 & ANXA7 & {$[65]$} \\
\hline Antileukoproteinase* & SLPI_HUMAN & P03973 & SLPI & {$[52]$} \\
\hline Apelin receptor* & APJ_HUMAN & P35414 & APLNR & {$[60]$} \\
\hline Apolipoprotein A-IV* & APOA4_HUMAN & P06727 & APOA4 & {$[52,65]$} \\
\hline Apolipoprotein B-100* & APOB_HUMAN & P04114 & APOB & {$[59,65]$} \\
\hline Apolipoprotein C-I* & APOC1_HUMAN & P02654 & APOC1 & {$[65]$} \\
\hline Apolipoprotein C-II* & APOC2_HUMAN & P02655 & APOC2 & {$[65]$} \\
\hline Apolipoprotein $\mathrm{E}^{*}$ & APOE_HUMAN & P02649 & APOE & {$[65]$} \\
\hline Apolipoprotein $\mathrm{M}^{*}$ & APOM_HUMAN & O95445 & APOM & {$[65]$} \\
\hline Apoptosis regulator $\mathrm{BAX}^{*}$ & BAX_HUMAN & Q07812 & BAX & {$[52]$} \\
\hline Artemin & ARTN_HUMAN & Q5T4W7 & ARTN & {$[52,60,65]$} \\
\hline
\end{tabular}


Table 1 (continued)

\begin{tabular}{|c|c|c|c|c|}
\hline Protein & Abbreviation & UniProtKB & Gene & Ref. \\
\hline Aspartyl/asparaginyl beta-hydroxylase* & ASPH_HUMAN & Q12797 & ASPH & {$[52,65]$} \\
\hline Basal cell adhesion molecule & BCAM_HUMAN & P50895 & BCAM & [57] \\
\hline BCL2/adenovirus E1B $19 \mathrm{kDa}$ protein-interacting protein $2 *$ & BNIP2_HUMAN & Q12982 & BNIP2 & {$[52,65]$} \\
\hline Beta-2-microglobulin* & B2MG_HUMAN & P61769 & $\mathrm{B} 2 \mathrm{M}$ & {$[65]$} \\
\hline Beta-Ala-His dipeptidase* & CNDP1_HUMAN & Q96KN2 & CNDP1 & {$[52,65]$} \\
\hline Beta-defensin $1^{*}$ & DEFB1_HUMAN & P60022 & DEFB1 & {$[52]$} \\
\hline Beta-defensin 4A & DFB4A_HUMAN & $\mathrm{O} 15263$ & DEFB4A & {$[65]$} \\
\hline Beta-endorphin (Pro-opiomelanocortin)* & COLI_HUMAN & P01189 & POMC & {$[52,65]$} \\
\hline BMP-binding endothelial regulator protein* & BMPER_HUMAN & Q8N8U9 & BMPER & {$[52,60,65]$} \\
\hline Bone morphogenetic protein 1 & BMP1_HUMAN & P13497 & BMP1 & {$[57]$} \\
\hline Bone morphogenetic protein $3^{*}$ & BMP3_HUMAN & P12645 & BMP3 & {$[65]$} \\
\hline Bone morphogenetic protein $4 *$ & BMP4_HUMAN & P12644 & BMP4 & {$[52,65]$} \\
\hline Bone morphogenetic protein $5^{*}$ & BMP5_HUMAN & P22003 & BMP5 & {$[52]$} \\
\hline Bone morphogenetic protein $6^{*}$ & BMP6_HUMAN & P22004 & BMP6 & {$[65]$} \\
\hline Bone morphogenetic protein $7 *$ & BMP7_HUMAN & P18075 & BMP7 & {$[52,65]$} \\
\hline Bone morphogenetic protein $8 \mathrm{~B}^{*}$ & BMP8B_HUMAN & P34820 & BMP8B & [52] \\
\hline Bone morphogenetic protein receptor type-1A & BMR1A_HUMAN & P36894 & BMPR1A & {$[57]$} \\
\hline Bone morphogenetic protein receptor type-1B* & BMR1B_HUMAN & O00238 & BMPR1B & {$[65]$} \\
\hline Bone morphogenetic protein receptor type- 2 & BMPR2_HUMAN & Q13873 & BMPR2 & {$[57]$} \\
\hline Brain-derived neurotrophic factor* & BDNF_HUMAN & P23560 & BDNF & {$[65]$} \\
\hline CA 19-9 & - & - & $\begin{array}{l}\text { ST6GALNAC (partly } \\
\text { synthesized by) }\end{array}$ & {$[52]$} \\
\hline Cadherin-1 & CADH1_HUMAN & P12830 & CDH1 & {$[57]$} \\
\hline Cadherin-2 & CADH2_HUMAN & P19022 & $\mathrm{CDH} 2$ & {$[57]$} \\
\hline Cadherin-5 & CADH5_HUMAN & P33151 & CDH5 & [57] \\
\hline Cadherin-11 & CAD11_HUMAN & P55287 & CDH11 & {$[57]$} \\
\hline Cadherin-13 & CAD13_HUMAN & P55290 & $\mathrm{CDH} 13$ & {$[57]$} \\
\hline Cadherin-related family member 2 & CDHR2_HUMAN & Q9BYE9 & CDHR2 & {$[57]$} \\
\hline Cadherin-related family member 5 & CDHR5_HUMAN & Q9HBB8 & CDHR5 & {$[57]$} \\
\hline Calbindin & CALB1_HUMAN & P05937 & CALB1 & {$[52,65]$} \\
\hline Calcitonin & CALC_HUMAN & P01258 & CALCA & {$[52]$} \\
\hline Calreticulin & CALR_HUMAN & P27797 & CALR & {$[65]$} \\
\hline Calsyntenin-1 & CSTN1_HUMAN & O94985 & CLSTN1 & {$[65]$} \\
\hline Carboxypeptidase $\mathrm{N}$ subunit $2 *$ & CPN2_HUMAN & P22792 & CPN2 & {$[52,65]$} \\
\hline Carcinoembryonic antigen-related cell adhesion molecule $7 *$ & CEAM7_HUMAN & Q14002 & CEACAM7 & {$[65]$} \\
\hline Caspase-3 & CASP3_HUMAN & P42574 & CASP3 & {$[65]$} \\
\hline Caspase- 8 & CASP8_HUMAN & Q14790 & CASP8 & {$[52]$} \\
\hline Cathepsin B & CATB_HUMAN & P07858 & CTSB & {$[65]$} \\
\hline Cathepsin D & CATD_HUMAN & P07339 & CTSD & {$[65]$} \\
\hline C-C chemokine receptor type $1 *$ & CCR1_HUMAN & P32246 & CCR1 & {$[65]$} \\
\hline C-C chemokine receptor type $2 *$ & CCR2_HUMAN & P41597 & CCR2 & {$[65]$} \\
\hline C-C chemokine receptor type $3 *$ & CCR3_HUMAN & P51677 & CCR3 & {$[52]$} \\
\hline C-C chemokine receptor type $4^{*}$ & CCR4_HUMAN & P51679 & CCR4 & {$[65]$} \\
\hline C-C chemokine receptor type $5^{*}$ & CCR5_HUMAN & P51681 & CCR5 & {$[65]$} \\
\hline C-C chemokine receptor type $6^{*}$ & CCR6_HUMAN & P51684 & CCR6 & {$[65]$} \\
\hline C-C chemokine receptor type $7 *$ & CCR7_HUMAN & P32248 & CCR7 & {$[65]$} \\
\hline C-C chemokine receptor type $9 *$ & CCR9_HUMAN & P51686 & CCR9 & {$[65]$} \\
\hline C-C motif chemokine $1^{*}$ & CCL1_HUMAN & P22362 & CCL1 & {$[61,65]$} \\
\hline C-C motif chemokine $2 *$ & CCL2_HUMAN & P13500 & CCL2 & {$[52]$} \\
\hline
\end{tabular}


Table 1 (continued)

\begin{tabular}{|c|c|c|c|c|}
\hline Protein & Abbreviation & UniProtKB & Gene & Ref. \\
\hline C-C motif chemokine $3 *$ & CCL3_HUMAN & P10147 & CCL3 & {$[65]$} \\
\hline C-C motif chemokine $4 *$ & CCL4_HUMAN & P13236 & CCL4 & {$[52]$} \\
\hline C-C motif chemokine $5^{*}$ & CCL5_HUMAN & P13501 & CCL5 & {$[65]$} \\
\hline C-C motif chemokine $7 *$ & CCL7_HUMAN & P80098 & CCL7 & {$[61]$} \\
\hline C-C motif chemokine $8^{*}$ & CCL8_HUMAN & P80075 & CCL8 & {$[61,65]$} \\
\hline C-C motif chemokine $13^{*}$ & CCL13_HUMAN & Q99616 & CCL13 & {$[61,65]$} \\
\hline C-C motif chemokine $14 *$ & CCL14_HUMAN & Q16627 & CCL14 & {$[52,60,65]$} \\
\hline C-C motif chemokine $16^{*}$ & CCL16_HUMAN & O15467 & CCL16 & {$[65]$} \\
\hline C-C motif chemokine $18^{*}$ & CCL18_HUMAN & P55774 & CCL18 & [52] \\
\hline C-C motif chemokine $19^{*}$ & CCL19_HUMAN & Q99731 & CCL19 & [52] \\
\hline C-C motif chemokine $21^{*}$ & CCL21_HUMAN & O00585 & CCL21 & {$[65]$} \\
\hline $\mathrm{C}-\mathrm{C}$ motif chemokine $22^{*}$ & CCL22_HUMAN & O00626 & CCL22 & {$[65]$} \\
\hline C-C motif chemokine $26^{*}$ & CCL26_HUMAN & Q9Y258 & CCL26 & {$[65]$} \\
\hline C-C motif chemokine $27 *$ & CCL27_HUMAN & Q9Y4X3 & CCL27 & {$[52]$} \\
\hline C-C motif chemokine $28^{*}$ & CCL28_HUMAN & Q9NRJ3 & CCL28 & {$[52,60]$} \\
\hline CD166 antigen & CD166_HUMAN & Q13740 & ALCAM & {$[52,65]$} \\
\hline CD27 antigen & CD27_HUMAN & P26842 & $\mathrm{CD} 27$ & {$[65]$} \\
\hline CD44 antigen & CD44_HUMAN & P16070 & CD44 & {$[12,57,65]$} \\
\hline CD59 glycoprotein* & CD59_HUMAN & P13987 & CD59 & {$[52]$} \\
\hline CD63 antigen & CD63_HUMAN & P08962 & CD63 & {$[12]$} \\
\hline Cdc42-interacting protein 4 & CIP4_HUMAN & Q15642 & TRIP10 & {$[57]$} \\
\hline Cell division control protein 42 homolog & CDC42_HUMAN & P60953 & $\mathrm{CDC} 42$ & {$[57]$} \\
\hline Cerberus & CER1_HUMAN & O95813 & CER1 & {$[65]$} \\
\hline Ceruloplasmin & CERU_HUMAN & P00450 & $\mathrm{CP}$ & {$[52,65]$} \\
\hline Chitinase-3-like protein $1 *$ & CH3L1_HUMAN & P36222 & CHI3L1 & {$[52,65]$} \\
\hline Chordin-like protein $2 *$ & CRDL2_HUMAN & Q6WN34 & CHRDL2 & {$[52]$} \\
\hline Ciliary neurotrophic factor receptor subunit alpha* & CNTFR_HUMAN & P26992 & CNTFR & {$[52]$} \\
\hline Ciliary neurotrophic factor* & CNTF_HUMAN & P26441 & CNTF & {$[52,65]$} \\
\hline Clusterin & CLUS_HUMAN & P10909 & CLU & {$[52]$} \\
\hline Coagulation factor XIII A chain & F13A_HUMAN & P00488 & F13A1 & {$[52]$} \\
\hline Coagulation factor XIII B chain & F13B_HUMAN & P05160 & F13B & {$[65]$} \\
\hline Collagen alpha-1(I) chain & CO1A1_HUMAN & P02452 & COL1A1 & {$[57]$} \\
\hline Collagen alpha-1(III) chain & CO3A1_HUMAN & P02461 & COL3A1 & {$[57]$} \\
\hline Collagen alpha-1(IV) chain & CO4A1_HUMAN & P02462 & COL4A1 & {$[57]$} \\
\hline Collagen alpha-1(V) chain & CO5A1_HUMAN & P20908 & COL5A1 & {$[57]$} \\
\hline Collagen alpha-1(VI) chain & CO6A1_HUMAN & P12109 & COL6A1 & {$[57]$} \\
\hline Collagen alpha-1(VII) chain & CO7A1_HUMAN & Q02388 & COL7A1 & {$[57]$} \\
\hline Collagen alpha-1(XII) chain & COCA1_HUMAN & Q99715 & COL12A1 & {$[57]$} \\
\hline Collagen alpha- $1(\mathrm{XV})$ chain & COFA1_HUMAN & P39059 & COL15A1 & {$[57]$} \\
\hline Collagen alpha-2(I) chain & CO1A2_HUMAN & P08123 & COL1A2 & {$[57]$} \\
\hline Collagen alpha-2(IV) chain & CO4A2_HUMAN & P08572 & COL4A2 & {$[57]$} \\
\hline Collagen alpha-2(V) chain & CO5A2_HUMAN & P05997 & COL5A2 & {$[57]$} \\
\hline Collagen alpha-2(VI) chain & CO6A2_HUMAN & P12110 & COL6A2 & {$[57]$} \\
\hline Collagen alpha-3(VI) chain & CO6A3_HUMAN & P12111 & COL6A3 & {$[57]$} \\
\hline Collagenase $3^{*}$ & MMP13_HUMAN & P45452 & MMP13 & {$[65]$} \\
\hline Complement $\mathrm{C} 2 *$ & CO2_HUMAN & P06681 & $\mathrm{C} 2$ & {$[52,65]$} \\
\hline Complement $\mathrm{C} 3 *$ & CO3_HUMAN & P01024 & $\mathrm{C} 3$ & {$[65]$} \\
\hline Complement C5* & CO5_HUMAN & P01031 & $\mathrm{C} 5$ & {$[65]$} \\
\hline Complement factor H-related protein $2 *$ & FHR2_HUMAN & P36980 & CFHR2 & {$[65]$} \\
\hline
\end{tabular}


Table 1 (continued)

\begin{tabular}{|c|c|c|c|c|}
\hline Protein & Abbreviation & UniProtKB & Gene & Ref. \\
\hline Corticosteroid 11-beta-dehydrogenase isozyme $1^{*}$ & DHI1_HUMAN & P28845 & HSD11B1 & {$[65]$} \\
\hline Corticosteroid-binding globulin & CBG_HUMAN & P08185 & SERPINA6 & {$[52]$} \\
\hline C-peptide*** & - & - & INS & {$[52,65]$} \\
\hline C-reactive protein* & CRP_HUMAN & P02741 & CRP & {$[65]$} \\
\hline Creatine kinase B-type* & KCRB_HUMAN & P12277 & $\mathrm{CKB}$ & {$[52,65]$} \\
\hline CREB-binding protein* & CBP_HUMAN & Q92793 & CREBBP & {$[52]$} \\
\hline Cryptic protein & CFC1_HUMAN & P0CG37 & $\mathrm{CFC} 1$ & {$[52,65]$} \\
\hline C-X-C chemokine receptor type $6^{*}$ & CXCR6_HUMAN & O00574 & CXCR6 & {$[65]$} \\
\hline $\mathrm{C}-\mathrm{X}-\mathrm{C}$ motif chemokine $2^{*}$ & CXCL2_HUMAN & P19875 & CXCL2 & {$[52,60,65]$} \\
\hline C-X-C motif chemokine $5^{*}$ & CXCL5_HUMAN & P42830 & CXCL5 & {$[65]$} \\
\hline C-X-C motif chemokine $9 *$ & CXCL9_HUMAN & Q07325 & CXCL9 & {$[52]$} \\
\hline C-X-C motif chemokine $10^{*}$ & CXL10_HUMAN & P02778 & CXCL10 & {$[65]$} \\
\hline C-X-C motif chemokine $11^{*}$ & CXL11_HUMAN & O14625 & CXCL11 & {$[61,65]$} \\
\hline C-X-C motif chemokine $16^{*}$ & CXL16_HUMAN & Q9H2A7 & CXCL16 & {$[61,65]$} \\
\hline Cyclin-dependent kinase inhibitor $1^{*}$ & CDN1A_HUMAN & P38936 & CDKN1A & {$[65]$} \\
\hline Cystatin A & CYTA_HUMAN & P01040 & CSTA & {$[65]$} \\
\hline Cytokine receptor common subunit gamma* & IL2RG_HUMAN & P31785 & IL2RG & {$[52,65]$} \\
\hline Cytoplasmic tyrosine-protein kinase BMX* & BMX_HUMAN & P51813 & BMX & {$[65]$} \\
\hline Cytotoxic and regulatory $\mathrm{T}$ cell molecule* & CRTAM_HUMAN & O95727 & CRTAM & {$[65]$} \\
\hline Cytotoxic T lymphocyte protein $4 *$ & CTLA4_HUMAN & P16410 & CTLA4 & {$[52,65]$} \\
\hline DAN domain family member $5^{*}$ & DAND5_HUMAN & Q8N907 & DAND5 & {$[65]$} \\
\hline Decorin & PGS2_HUMAN & P07585 & $\mathrm{DCN}$ & {$[65]$} \\
\hline Dentin matrix acidic phosphoprotein $1^{*}$ & DMP1_HUMAN & Q13316 & DMP1 & {$[65]$} \\
\hline Dermcidin & DCD_HUMAN & P81605 & DCD & {$[59]$} \\
\hline Dickkopf-related protein $1 *$ & DKK1_HUMAN & O94907 & DKK1 & {$[65]$} \\
\hline Dickkopf-related protein $3 *$ & DKK3_HUMAN & Q9UBP4 & DKK3 & {$[65]$} \\
\hline Dickkopf-related protein $4^{*}$ & DKK4_HUMAN & Q9UBT3 & DKK4 & {$[52]$} \\
\hline Discoidin domain-containing receptor $2 *$ & DDR2_HUMAN & Q16832 & DDR2 & {$[52]$} \\
\hline Discoidin, CUB and LCCL domain-containing protein $2 *$ & DCBD2_HUMAN & Q96PD2 & DCBLD2 & {$[65]$} \\
\hline Echinoderm microtubule-associated protein-like $2 *$ & EMAL2_HUMAN & O95834 & EML2 & {$[52,65]$} \\
\hline Ectodysplasin-A* & EDA_HUMAN & Q92838 & EDA & {$[60,65]$} \\
\hline $\begin{array}{l}\text { Ectonucleotide pyrophosphatase/ } \\
\text { phosphodiesterase family member } 2 *\end{array}$ & ENPP2_HUMAN & Q13822 & ENPP2 & {$[52]$} \\
\hline $\begin{array}{l}\text { EGF-like repeat and discoidin I-like domain-containing } \\
\text { protein } 3\end{array}$ & EDIL3_HUMAN & $\mathrm{O} 43854$ & EDIL3 & {$[57]$} \\
\hline Elongation factor 1-alpha 1 & EF1A1_HUMAN & P68104 & EEF1A1 & {$[12]$} \\
\hline Elongation factor $2^{*}$ & EF2_HUMAN & P13639 & EEF2 & {$[12]$} \\
\hline Embryonic growth/differentiation factor $1^{*}$ & GDF1_HUMAN & P27539 & GDF1 & {$[52]$} \\
\hline Endoglin & EGLN_HUMAN & P17813 & ENG & {$[52]$} \\
\hline Endostatin (cleaved from Collagen alpha-1(XVIII) chain) & COIA1_HUMAN & P39060 & COL18A1 & {$[52,57,60,65]$} \\
\hline Endothelial cell-selective adhesion molecule* & ESAM_HUMAN & Q96AP7 & ESAM & {$[65]$} \\
\hline Endothelin-1 receptor* & EDNRA_HUMAN & P25101 & EDNRA & {$[52,65]$} \\
\hline Eotaxin & CCL11_HUMAN & P51671 & CCL11 & {$[65]$} \\
\hline Ephrin type-A receptor $4 *$ & EPHA4_HUMAN & P54764 & EPHA4 & {$[52]$} \\
\hline Ephrin type-A receptor $6^{*}$ & EPHA6_HUMAN & Q9UF33 & EPHA6 & {$[65]$} \\
\hline Ephrin type-A receptor $8^{*}$ & EPHA8_HUMAN & P29322 & EPHA8 & {$[65]$} \\
\hline Ephrin type-B receptor $4^{*}$ & EPHB4_HUMAN & P54760 & EPHB4 & {$[65]$} \\
\hline Epidermal growth factor receptor* & EGFR_HUMAN & P00533 & EGFR & {$[57,65]$} \\
\hline Epidermal growth factor receptor substrate 15 -like 1 & EP15R_HUMAN & Q9UBC2 & EPS15L1 & {$[57]$} \\
\hline
\end{tabular}


Table 1 (continued)

\begin{tabular}{|c|c|c|c|c|}
\hline Protein & Abbreviation & UniProtKB & Gene & Ref. \\
\hline Epithelial cell adhesion molecule* & EPCAM_HUMAN & P16422 & EPCAM & {$[65]$} \\
\hline Erythropoietin & EPO_HUMAN & P01588 & EPO & {$[52]$} \\
\hline Erythropoietin receptor & EPOR_HUMAN & P19235 & EPOR & {$[65]$} \\
\hline E-Selectin & LYAM2_HUMAN & P16581 & SELE & {$[52]$} \\
\hline EVI5-like protein & EVI5L_HUMAN & Q96CN4 & EVI5L & {$[52]$} \\
\hline FAS-associated death domain protein* & FADD_HUMAN & Q13158 & FADD & {$[65]$} \\
\hline Fatty acid-binding protein 5 & FABP5_HUMAN & Q01469 & FABP5 & {$[59]$} \\
\hline Ferritin light chain* & FRIL_HUMAN & P02792 & FTL & {$[65]$} \\
\hline Fetuin-B & FETUB_HUMAN & Q9UGM5 & FETUB & {$[65]$} \\
\hline Fibrinogen-like protein $1 *$ & FGL1_HUMAN & Q08830 & FGL1 & {$[52,65]$} \\
\hline Fibrinopeptide A (cleaved from Fibrinogen alpha chain) & FIBA_HUMAN & P02671 & FGA & {$[52]$} \\
\hline Fibroblast growth factor $2^{*}$ & FGF2_HUMAN & P09038 & FGF2 & {$[57,65]$} \\
\hline Fibroblast growth factor $4 *$ & FGF4_HUMAN & P08620 & FGF4 & {$[61]$} \\
\hline Fibroblast growth factor $5^{*}$ & FGF5_HUMAN & P12034 & FGF5 & {$[52]$} \\
\hline Fibroblast growth factor $6^{*}$ & FGF6_HUMAN & P10767 & FGF6 & {$[65]$} \\
\hline Fibroblast growth factor $8^{*}$ & FGF8_HUMAN & P55075 & FGF8 & {$[65]$} \\
\hline Fibroblast growth factor $10^{*}$ & FGF10_HUMAN & $\mathrm{O} 15520$ & FGF10 & {$[52]$} \\
\hline Fibroblast growth factor $11^{*}$ & FGF11_HUMAN & Q92914 & FGF11 & {$[52]$} \\
\hline Fibroblast growth factor $12^{*}$ & FGF12_HUMAN & P61328 & FGF12 & {$[65]$} \\
\hline Fibroblast growth factor $13^{*}$ & FGF13_HUMAN & Q92913 & FGF13 & {$[52]$} \\
\hline Fibroblast growth factor $16^{*}$ & FGF16_HUMAN & O43320 & FGF16 & {$[52]$} \\
\hline Fibroblast growth factor $17^{*}$ & FGF17_HUMAN & O60258 & FGF17 & {$[52,65]$} \\
\hline Fibroblast growth factor $18^{*}$ & FGF18_HUMAN & O76093 & FGF18 & {$[52,65]$} \\
\hline Fibroblast growth factor $20^{*}$ & FGF20_HUMAN & Q9NP95 & FGF20 & {$[52,65]$} \\
\hline Fibroblast growth factor $21^{*}$ & FGF21_HUMAN & Q9NSA1 & FGF21 & {$[65]$} \\
\hline Fibroblast growth factor receptor 1 & FGFR1_HUMAN & P11362 & FGFR1 & {$[57]$} \\
\hline Fibroblast growth factor receptor $3 *$ & FGFR3_HUMAN & P22607 & FGFR3 & {$[65]$} \\
\hline Fibroblast growth factor receptor 4 & FGFR4_HUMAN & P22455 & FGFR4 & {$[57]$} \\
\hline Fibroblast growth factor-binding protein $1^{*}$ & FGFP1_HUMAN & Q14512 & FGFBP1 & {$[65]$} \\
\hline Fibronectin & FINC_HUMAN & P02751 & FN1 & {$[52,57]$} \\
\hline Filaggrin-2 & FILA2_HUMAN & Q5D862 & FLG2 & [59] \\
\hline Follistatin & FST_HUMAN & P19883 & FST & {$[52,61,65]$} \\
\hline Follistatin-related protein $3 *$ & FSTL3_HUMAN & O95633 & FSTL3 & {$[65]$} \\
\hline Forkhead box protein N3* & FOXN3_HUMAN & O00409 & FOXN3 & {$[52]$} \\
\hline Frizzled-1 & FZD1_HUMAN & Q9UP38 & FZD1 & {$[52,57,65]$} \\
\hline Frizzled-3 & FZD3_HUMAN & Q9NPG1 & FZD3 & {$[52,65]$} \\
\hline Frizzled-6 & FZD6_HUMAN & O60353 & FZD6 & {$[57]$} \\
\hline Frizzled-7 & FZD7_HUMAN & O75084 & FZD7 & {$[65]$} \\
\hline Fructose-bisphosphate aldolase $\mathrm{A}^{*}$ & ALDOA_HUMAN & P04075 & ALDOA & {$[52]$} \\
\hline Fructose-bisphosphate aldolase B & ALDOB_HUMAN & P05062 & ALDOB & {$[65]$} \\
\hline Fructose-bisphosphate aldolase $\mathrm{C}^{*}$ & ALDOC_HUMAN & P09972 & ALDOC & {$[52,65]$} \\
\hline Furin & FURIN_HUMAN & P09958 & FURIN & {$[65]$} \\
\hline Galanin peptides & GALA_HUMAN & P22466 & GAL & {$[52]$} \\
\hline Galectin-10* & LEG10_HUMAN & Q05315 & CLC & {$[52,65]$} \\
\hline Galectin-3 & LEG3_HUMAN & P17931 & LGALS3 & {$[52,65]$} \\
\hline Gamma-Thrombin (cleaved from prothrombin) & THRB_HUMAN & P00734 & $\mathrm{F} 2$ & {$[65]$} \\
\hline GATA-type zinc finger protein $1 *$ & ZGLP1_HUMAN & P0C6A0 & ZGLP1 & {$[52]$} \\
\hline GDNF family receptor alpha-3* & GFRA3_HUMAN & O60609 & GFRA3 & {$[52]$} \\
\hline Geminin* & GEMI_HUMAN & O75496 & GMNN & {$[65]$} \\
\hline
\end{tabular}


Table 1 (continued)

\begin{tabular}{|c|c|c|c|c|}
\hline Protein & Abbreviation & UniProtKB & Gene & Ref. \\
\hline Glial cell line-derived neurotrophic factor* & GDNF_HUMAN & P39905 & GDNF & {$[65]$} \\
\hline Glutathione peroxidase $1^{*}$ & GPX1_HUMAN & P07203 & GPX1 & {$[65]$} \\
\hline Glutathione peroxidase $3 *$ & GPX3_HUMAN & P22352 & GPX3 & {$[65]$} \\
\hline Glyceraldehyde 3-phosphate dehydrogenase & G3P_HUMAN & P04406 & GAPDH & {$[12]$} \\
\hline Glycogen phosphorylase, brain form* & PYGB_HUMAN & P11216 & PYGB & {$[65]$} \\
\hline Glycoprotein hormones alpha chain* & GLHA_HUMAN & P01215 & CGA & {$[52]$} \\
\hline Glypican-3 & GPC3_HUMAN & P51654 & GPC3 & {$[60]$} \\
\hline Glypican-5 & GPC5_HUMAN & P78333 & GPC5 & {$[65]$} \\
\hline Granulocyte colony-stimulating factor* & CSF3_HUMAN & P09919 & CSF3 & {$[52,60,61,65]$} \\
\hline $\begin{array}{l}\text { Granulocyte-macrophage colony-stimulating factor } \\
\text { receptor subunit alpha* }\end{array}$ & CSF2R_HUMAN & P15509 & CSF2RA & {$[52,65]$} \\
\hline Granulocyte-macrophage colony-stimulating factor* & CSF2_HUMAN & P04141 & CSF2 & {$[52,61]$} \\
\hline Granzyme A & GRAA_HUMAN & P12544 & GZMA & {$[52,65]$} \\
\hline Gremlin-1 & GREM1_HUMAN & O60565 & GREM1 & {$[52]$} \\
\hline $\begin{array}{l}\text { Growth arrest and DNA damage-inducible protein } \\
\text { GADD45 alpha* }\end{array}$ & GA45A_HUMAN & P24522 & GADD45A & {$[52]$} \\
\hline Growth factor receptor-bound protein 2 & GRB2_HUMAN & P62993 & GRB2 & {$[57]$} \\
\hline Growth/differentiation factor $2 *$ & GDF2_HUMAN & Q9UK05 & GDF2 & {$[65]$} \\
\hline Growth/differentiation factor $3 *$ & GDF3_HUMAN & Q9NR23 & GDF3 & {$[52,65]$} \\
\hline Growth/differentiation factor $5^{*}$ & GDF5_HUMAN & P43026 & GDF5 & {$[52,65]$} \\
\hline Growth/differentiation factor $8^{*}$ & GDF8_HUMAN & O14793 & MSTN & {$[52]$} \\
\hline Growth/differentiation factor $9^{*}$ & GDF9_HUMAN & O60383 & GDF9 & {$[52,65]$} \\
\hline Growth/differentiation factor $11 *$ & GDF11_HUMAN & O95390 & GDF11 & {$[52,57,65]$} \\
\hline $\begin{array}{l}\text { Guanine nucleotide-binding protein } \mathrm{G}(\mathrm{I}) / \mathrm{G}(\mathrm{S}) / \mathrm{G}(\mathrm{O}) \\
\text { subunit gamma-12 }\end{array}$ & GBG12_HUMAN & Q9UBI6 & GNG12 & {$[57]$} \\
\hline Guanine nucleotide-binding protein subunit alpha-13 & GNA13_HUMAN & Q14344 & GNA13 & {$[57]$} \\
\hline Haptoglobin & HPT_HUMAN & P00738 & $\mathrm{HP}$ & {$[52]$} \\
\hline HCR / CRAM-A/B*** & - & - & CCHCR1 & {$[52,65]$} \\
\hline Heat shock protein $70 \mathrm{kDa}^{* *}$ & - & - & - & {$[12]$} \\
\hline Heat shock protein $90 \mathrm{kDa} * *$ & - & - & - & {$[12]$} \\
\hline Heat shock protein $105 \mathrm{kDa} *$ & HS105_HUMAN & Q92598 & HSPH1 & {$[12]$} \\
\hline Heat shock protein beta-1* & HSPB1_HUMAN & P04792 & HSPB1_HUMAN & {$[12,52,65]$} \\
\hline Hepatocyte growth factor activator & HGFA_HUMAN & Q04756 & HGFAC & {$[57]$} \\
\hline Hepatocyte growth factor receptor* & MET_HUMAN & P08581 & MET & {$[52]$} \\
\hline $\begin{array}{l}\text { Hepatocyte growth factor-like protein alpha chain (cleaved } \\
\text { from hepatocyte growth factor-like protein)* }\end{array}$ & HGFL_HUMAN & P26927 & MST1 & {$[52]$} \\
\hline Hepatocyte growth factor-regulated tyrosine kinase substrate & HGS_HUMAN & O14964 & HGS & {$[57]$} \\
\hline Hepcidin & HEPC_HUMAN & P81172 & HAMP & {$[65]$} \\
\hline Histone H4 & H4_HUMAN & P62805 & $\mathrm{H} 4 \mathrm{C} 1$ & {$[59]$} \\
\hline HLA class II histocompatibility antigen gamma chain* & HG2A_HUMAN & P04233 & CD74 & {$[65]$} \\
\hline Homeobox protein NANOG* & NANOG_HUMAN & Q9H9S0 & NANOG & {$[65]$} \\
\hline Hornerin & HORN_HUMAN & Q86YZ3 & HRNR & {$[59]$} \\
\hline Inhibin beta A chain* & INHBA_HUMAN & P08476 & INHBA & {$[65]$} \\
\hline Inhibin beta B chain* & INHBB_HUMAN & P09529 & INHBB & {$[65]$} \\
\hline Inhibin beta $\mathrm{C}$ chain* & INHBC_HUMAN & P55103 & INHBC & {$[60]$} \\
\hline INSL3 $* * *$ & - & - & - & {$[65]$} \\
\hline Insulin receptor* & INSR_HUMAN & P06213 & INSR & {$[52,65]$} \\
\hline Insulin-degrading enzyme* & IDE_HUMAN & P14735 & IDE & {$[65]$} \\
\hline Insulin-like growth factor 1 receptor & IGF1R_HUMAN & P08069 & IGF1R & {$[57]$} \\
\hline Insulin-like growth factor I* & IGF1_HUMAN & P05019 & IGF1 & {$[65]$} \\
\hline
\end{tabular}


Table 1 (continued)

\begin{tabular}{|c|c|c|c|c|}
\hline Protein & Abbreviation & UniProtKB & Gene & Ref. \\
\hline Insulin-like growth factor-binding protein $1^{*}$ & IBP1_HUMAN & P08833 & IGFBP1 & {$[65]$} \\
\hline Insulin-like growth factor-binding protein 3 & IBP3_HUMAN & P17936 & IGFBP3 & {$[57]$} \\
\hline Insulin-like growth factor-binding protein $4^{*}$ & IBP4_HUMAN & P22692 & IGFBP4 & {$[52]$} \\
\hline Insulin-like growth factor-binding protein $5^{*}$ & IBP5_HUMAN & P24593 & IGFBP5 & {$[65]$} \\
\hline Insulin-like growth factor-binding protein $7 *$ & IBP7_HUMAN & Q16270 & IGFBP7 & {$[60,65]$} \\
\hline $\begin{array}{l}\text { Insulin-like growth factor-binding protein complex acid } \\
\text { labile subunit }\end{array}$ & ALS_HUMAN & P35858 & IGFALS & {$[57]$} \\
\hline Integrin alpha-1 & ITA1_HUMAN & P56199 & ITGA1 & {$[57]$} \\
\hline Integrin alpha-2 & ITA2_HUMAN & P17301 & ITGA2 & {$[57]$} \\
\hline Integrin alpha-3 & ITA3_HUMAN & P26006 & ITGA3 & {$[57]$} \\
\hline Integrin alpha-4 & ITA4_HUMAN & P13612 & ITGA4 & {$[57]$} \\
\hline Integrin alpha-5 & ITA5_HUMAN & P08648 & ITGA5 & {$[57]$} \\
\hline Integrin alpha-6 & ITA6_HUMAN & P23229 & ITGA6 & {$[57]$} \\
\hline Integrin alpha-7 & ITA7_HUMAN & Q13683 & ITGA7 & {$[57]$} \\
\hline Integrin alpha-10 & ITA10_HUMAN & O75578 & ITGA10 & {$[57]$} \\
\hline Integrin alpha-11 & ITA11_HUMAN & Q9UKX5 & ITGA11 & {$[57]$} \\
\hline Integrin alpha-M* & ITAM_HUMAN & P11215 & ITGAM & {$[52]$} \\
\hline Integrin alpha- $\mathrm{V}$ & ITAV_HUMAN & P06756 & ITGAV & {$[52,57,65]$} \\
\hline Integrin beta- 1 & ITB1_HUMAN & P05556 & ITGB1 & {$[57]$} \\
\hline Integrin beta-1-binding protein 1 & ITBP1_HUMAN & $\mathrm{O} 14713$ & ITGB1BP1 & {$[57]$} \\
\hline Integrin beta-3 & ITB3_HUMAN & P05106 & ITGB3 & {$[57]$} \\
\hline Integrin beta- 5 & ITB5_HUMAN & P18084 & ITGB5 & {$[57]$} \\
\hline Integrin-linked protein kinase & ILK_HUMAN & Q13418 & ILK & {$[57]$} \\
\hline Inter-alpha-trypsin inhibitor heavy chain $\mathrm{H} 2$ & ITIH2_HUMAN & P19823 & ITIH2 & {$[59]$} \\
\hline Intercellular adhesion molecule 1 & ICAM1_HUMAN & P05362 & ICAM1 & {$[57]$} \\
\hline Intercellular adhesion molecule $2 *$ & ICAM2_HUMAN & P13598 & ICAM2 & {$[57,65]$} \\
\hline Interferon beta* & IFNB_HUMAN & P01574 & IFNB1 & {$[65]$} \\
\hline Interferon gamma* & IFNG_HUMAN & P01579 & IFNG & {$[52,65]$} \\
\hline Interferon lambda- $1^{*}$ & IFNL1_HUMAN & Q8IU54 & IFNL1 & {$[65]$} \\
\hline Interferon lambda- $2^{*}$ & IFNL2_HUMAN & Q8IZJ0 & IFNL2 & {$[65]$} \\
\hline Interferon regulatory factor $6^{*}$ & IRF6_HUMAN & O14896 & IRF6 & {$[52]$} \\
\hline Interleukin-1 alpha* & IL1A_HUMAN & P01583 & IL1A & {$[52,60,65]$} \\
\hline Interleukin-1 beta* & IL1B_HUMAN & P01584 & IL1B & {$[61]$} \\
\hline Interleukin-1 family member $10^{*}$ & IL1FA_HUMAN & Q8WWZ1 & IL1F10 & {$[52,65]$} \\
\hline Interleukin-1 receptor accessory protein-like $1^{*}$ & IRPL1_HUMAN & Q9NZN1 & IL1RAPL1 & {$[52,65]$} \\
\hline Interleukin-1 receptor type $1^{*}$ & IL1R1_HUMAN & P14778 & IL1R1 & {$[52]$} \\
\hline Interleukin-1 receptor type $2 *$ & IL1R2_HUMAN & P27930 & IL1R2 & {$[52]$} \\
\hline Interleukin-1 receptor-like $1 *$ & ILRL1_HUMAN & Q01638 & IL1RL1 & {$[52]$} \\
\hline Interleukin-1 receptor-like $2 *$ & ILRL2_HUMAN & Q9HB29 & IL1RL2 & {$[52]$} \\
\hline Interleukin- $2^{*}$ & IL2_HUMAN & P60568 & IL2 & {$[52]$} \\
\hline Interleukin-2 receptor subunit alpha* & IL2RA_HUMAN & P01589 & IL2RA & {$[65]$} \\
\hline Interleukin-2 receptor subunit beta* & IL2RB_HUMAN & P14784 & IL2RB & {$[52]$} \\
\hline Interleukin- $4 *$ & IL4_HUMAN & P05112 & IL4 & {$[61]$} \\
\hline Interleukin- $5 *$ & IL5_HUMAN & P05113 & IL5 & {$[52]$} \\
\hline Interleukin-6* & IL6_HUMAN & P05231 & IL6 & {$[52,62]$} \\
\hline Interleukin- $7 *$ & IL7_HUMAN & $\mathrm{P} 13232$ & IL7 & {$[52,65]$} \\
\hline Interleukin-7 receptor subunit alpha* & IL7RA_HUMAN & P16871 & IL7R & {$[65]$} \\
\hline Interleukin- $8^{*}$ & IL8_HUMAN & P10145 & CXCL8 & {$[52,65]$} \\
\hline Interleukin-9* & IL9_HUMAN & P15248 & IL9 & {$[52,65]$} \\
\hline
\end{tabular}


Table 1 (continued)

\begin{tabular}{|c|c|c|c|c|}
\hline Protein & Abbreviation & UniProtKB & Gene & Ref. \\
\hline Interleukin-10* & IL10_HUMAN & P22301 & IL10 & {$[52,61]$} \\
\hline Interleukin-10 receptor subunit alpha* & I10R1_HUMAN & Q13651 & IL10RA & {$[52]$} \\
\hline Interleukin-11* & IL11_HUMAN & P20809 & IL11 & {$[52]$} \\
\hline Interleukin-12 subunit alpha* & IL12A_HUMAN & P29459 & IL12A & [61] \\
\hline Interleukin-12 subunit beta* & IL12B_HUMAN & P29460 & IL12B & {$[61]$} \\
\hline Interleukin-13 receptor subunit alpha- $1 *$ & I13R1_HUMAN & P78552 & IL13RA1 & {$[52,65]$} \\
\hline Interleukin-13 receptor subunit alpha-2* & I13R2_HUMAN & Q14627 & IL13RA2 & {$[65]$} \\
\hline Interleukin-13* & IL13_HUMAN & P35225 & IL13 & {$[52]$} \\
\hline Interleukin-15* & IL15_HUMAN & P40933 & IL15 & {$[52]$} \\
\hline Interleukin-17 receptor $\mathrm{B}^{*}$ & I17RB_HUMAN & Q9NRM6 & IL17RB & {$[52,65]$} \\
\hline Interleukin-17 receptor $\mathrm{C}^{*}$ & I17RC_HUMAN & Q8NAC3 & IL17RC & {$[52]$} \\
\hline Interleukin-17A* & IL17_HUMAN & Q16552 & IL17A & {$[52,65]$} \\
\hline Interleukin-17C* & IL17C_HUMAN & Q9P0M4 & IL17C & {$[65]$} \\
\hline Interleukin-19* & IL19_HUMAN & Q9UHD0 & IL19 & {$[65]$} \\
\hline Interleukin-20 receptor subunit alpha* & I20RA_HUMAN & Q9UHF4 & IL20RA & {$[52]$} \\
\hline Interleukin-21 receptor* & IL21R_HUMAN & Q9HBE5 & IL21R & {$[65]$} \\
\hline Interleukin-21* & IL21_HUMAN & Q9HBE4 & IL21 & {$[52,65]$} \\
\hline Interleukin-23 receptor* & IL23R_HUMAN & Q5VWK5 & IL23R & {$[65]$} \\
\hline Interleukin-23 subunit alpha* & IL23A_HUMAN & Q9NPF7 & IL23A & {$[52,65]$} \\
\hline Interleukin-24* & IL24_HUMAN & Q13007 & IL24 & {$[65]$} \\
\hline Interleukin-27 subunit alpha* & IL27A_HUMAN & Q8NEV9 & IL27 & {$[65]$} \\
\hline Interleukin-36 gamma* & IL36G_HUMAN & Q9NZH8 & IL36G & {$[65]$} \\
\hline Interleukin-36 receptor antagonist protein* & I36RA_HUMAN & Q9UBH0 & IL36RN & {$[65]$} \\
\hline Interstitial collagenase* & MMP1_HUMAN & P03956 & MMP1 & {$[52,61]$} \\
\hline Islet amyloid polypeptide* & IAPP_HUMAN & P10997 & IAPP & {$[52,65]$} \\
\hline Junctional adhesion molecule $\mathrm{C}$ & JAM3_HUMAN & Q9BX67 & JAM3 & {$[57]$} \\
\hline Junctional adhesion molecule-like* & JAML_HUMAN & Q86YT9 & JAML & {$[65]$} \\
\hline Kallikrein 2 & KLK2_HUMAN & P20151 & KLK2 & {$[52]$} \\
\hline Kallikrein 11 & KLK11_HUMAN & Q9UBX7 & KLK11 & {$[65]$} \\
\hline Keratin, type I cytoskeletal 19* & K1C19_HUMAN & P08727 & KRT19 & {$[52,65]$} \\
\hline Kremen protein $1 *$ & KREM1_HUMAN & Q96MU8 & KREMEN1 & {$[52]$} \\
\hline Kremen protein $2 *$ & KREM2_HUMAN & Q8NCW0 & KREMEN2 & {$[60,65]$} \\
\hline Lactadherin* & MFGM_HUMAN & Q08431 & MFGE8 & {$[60]$} \\
\hline Lactotransferrin* & TRFL_HUMAN & P02788 & LTF & {$[52,59]$} \\
\hline Lactoylglutathione lyase* & LGUL_HUMAN & Q04760 & GLO1 & {$[65]$} \\
\hline Laminin subunit alpha-1 & LAMA1_HUMAN & P25391 & LAMA1 & {$[57]$} \\
\hline Laminin subunit alpha-2 & LAMA2_HUMAN & P24043 & LAMA2 & {$[57]$} \\
\hline Laminin subunit alpha-4 & LAMA4_HUMAN & Q16363 & LAMA4 & {$[57]$} \\
\hline Laminin subunit alpha-5 & LAMA5_HUMAN & $\mathrm{O} 15230$ & LAMA5 & {$[57]$} \\
\hline Laminin subunit beta-1 & LAMB1_HUMAN & P07942 & LAMB1 & {$[57]$} \\
\hline Laminin subunit beta- 2 & LAMB2_HUMAN & P55268 & LAMB2 & {$[57]$} \\
\hline Laminin subunit gamma-1 & LAMC1_HUMAN & P11047 & LAMC1 & {$[57]$} \\
\hline Latent-transforming growth factor beta-binding protein 1 & LTBP1_HUMAN & Q14766 & LTBP1 & {$[57]$} \\
\hline Layilin & LAYN_HUMAN & Q6UX15 & LAYN & {$[65]$} \\
\hline Leucine-rich alpha-2-glycoprotein* & A2GL_HUMAN & P02750 & LRG1 & {$[52,65]$} \\
\hline Leukocyte surface antigen CD47 & CD47_HUMAN & Q08722 & CD47 & {$[57]$} \\
\hline Lipopolysaccharide-binding protein* & LBP_HUMAN & P18428 & LBP & {$[65]$} \\
\hline L-lactate dehydrogenase A chain* & LDHA_HUMAN & P00338 & LDHA & {$[12]$} \\
\hline Low affinity immunoglobulin epsilon Fc receptor* & FCER2_HUMAN & P06734 & FCER2 & {$[65]$} \\
\hline
\end{tabular}


Table 1 (continued)

\begin{tabular}{|c|c|c|c|c|}
\hline Protein & Abbreviation & UniProtKB & Gene & Ref. \\
\hline Low-density lipoprotein receptor* & LDLR_HUMAN & P01130 & LDLR & {$[65]$} \\
\hline Low-density lipoprotein receptor-related protein $6^{*}$ & LRP6_HUMAN & O75581 & LRP6 & {$[60]$} \\
\hline L-Selectin & LYAM1_HUMAN & P14151 & SELL & {$[52]$} \\
\hline Lutropin-choriogonadotropic hormone receptor* & LSHR_HUMAN & P22888 & LHCGR & {$[52]$} \\
\hline Lymphocyte activation gene 3 protein* & LAG3_HUMAN & P18627 & LAG3 & {$[52]$} \\
\hline Lymphotoxin-alpha* & TNFB_HUMAN & P01374 & LTA & {$[52]$} \\
\hline Lymphotoxin-beta & TNFC_HUMAN & Q06643 & LTB & {$[65]$} \\
\hline Lysosome membrane protein $2 *$ & SCRB2_HUMAN & Q14108 & SCARB2 & {$[65]$} \\
\hline Lysosome-associated membrane glycoprotein $2 *$ & LAMP2_HUMAN & P13473 & LAMP2 & {$[12]$} \\
\hline Macrophage migration inhibitory factor* & MIF_HUMAN & P14174 & MIF & {$[65]$} \\
\hline Mammaglobin A & SG2A2_HUMAN & Q13296 & SCGB2A2 & {$[52]$} \\
\hline Mast/stem cell growth factor receptor Kit & KIT_HUMAN & P10721 & KIT & {$[57]$} \\
\hline Matrilysin* & MMP7_HUMAN & P09237 & MMP7 & {$[65]$} \\
\hline Matrix metalloproteinase- $9 *$ & MMP9_HUMAN & P14780 & MMP9 & {$[61,65]$} \\
\hline Matrix metalloproteinase-14* & MMP14_HUMAN & P50281 & MMP14 & {$[65]$} \\
\hline Matrix metalloproteinase-19* & MMP19_HUMAN & Q99542 & MMP19 & [52] \\
\hline Matrix metalloproteinase-20* & MMP20_HUMAN & O60882 & MMP20 & {$[52,60,65]$} \\
\hline Matrix metalloproteinase-24* & MMP24_HUMAN & Q9Y5R2 & MMP24 & {$[52,65]$} \\
\hline Megakaryocyte-associated tyrosine-protein kinase* & MATK_HUMAN & P42679 & MATK & [52] \\
\hline Metalloproteinase inhibitor 2 & TIMP2_HUMAN & P16035 & TIMP2 & {$[60]$} \\
\hline Metalloproteinase inhibitor $3 *$ & TIMP3_HUMAN & P35625 & TIMP3 & {$[65]$} \\
\hline MHC class I polypeptide-related sequence $\mathrm{A}^{*}$ & MICA_HUMAN & Q29983 & MICA & {$[65]$} \\
\hline Microglobulin*** & - & - & - & {$[65]$} \\
\hline Microtubule-associated tumor suppressor $1^{*}$ & MTUS1_HUMAN & Q9ULD2 & MTUS1 & {$[65]$} \\
\hline Mitogen-activated protein kinase 1 & MK01_HUMAN & P28482 & MAPK1 & {$[57]$} \\
\hline Mitogen-activated protein kinase 3 & MK03_HUMAN & P27361 & MAPK3 & {$[57]$} \\
\hline Monocyte differentiation antigen CD14* & CD14_HUMAN & P08571 & CD14 & {$[65]$} \\
\hline MSHa*** & - & - & MSX1 & {$[52]$} \\
\hline Mucin-1* & MUC1_HUMAN & P15941 & MUC1 & [65] \\
\hline Mucin-16* & MUC16_HUMAN & Q8WXI7 & MUC16 & {$[52,65]$} \\
\hline Mucosal addressin cell adhesion molecule 1 & $\begin{array}{l}\text { MADCA- } \\
\text { HUMAN }\end{array}$ & Q13477 & MADCAM1 & [57] \\
\hline Muscle, skeletal receptor tyrosine-protein kinase* & MUSK_HUMAN & O15146 & MUSK & {$[52]$} \\
\hline Myeloid-derived growth factor & MYDGF_HUMAN & Q969H8 & MYDGF & {$[57]$} \\
\hline Natriuretic peptides $\mathrm{B}^{*}$ & ANFB_HUMAN & P16860 & NPPB & {$[52]$} \\
\hline Natural killer cell receptor $2 \mathrm{~B} 4 *$ & CD244_HUMAN & Q9BZW8 & $\mathrm{CD} 244$ & {$[65]$} \\
\hline Neprilysin & NEP_HUMAN & P08473 & MME & {$[63]$} \\
\hline Netrin-1* & NET1_HUMAN & O95631 & NTN1 & {$[52]$} \\
\hline Netrin-G2 & NTNG2_HUMAN & Q96CW9 & NTNG2 & {$[52]$} \\
\hline Neural cell adhesion molecule $1 *$ & NCAM1_HUMAN & P13591 & NCAM1 & {$[65]$} \\
\hline Neural cell adhesion molecule L1-like protein & NCHL1_HUMAN & O00533 & CHL1 & {$[57]$} \\
\hline $\begin{array}{l}\text { Neuregulin-1 (cleaved form pro-neuregulin-1, } \\
\text { membrane-bound isoform) }\end{array}$ & NRG1_HUMAN & Q02297 & NRG1 & {$[52]$} \\
\hline $\begin{array}{l}\text { Neuregulin-2 (cleaved pro-neuregulin-2, membrane-bound } \\
\text { isoform)* }\end{array}$ & NRG2_HUMAN & O14511 & NRG2 & {$[52]$} \\
\hline $\begin{array}{l}\text { Neuregulin-3 (cleaved pro-neuregulin-3, membrane-bound } \\
\text { isoform)* }\end{array}$ & NRG3_HUMAN & P56975 & NRG3 & {$[52]$} \\
\hline Neurofibromin* & NF1_HUMAN & P21359 & NF1 & {$[52]$} \\
\hline Neurogenic differentiation factor $1 *$ & NDF1_HUMAN & Q13562 & NEUROD1 & {$[65]$} \\
\hline Neuronal pentraxin-1 & NPTX1_HUMAN & Q15818 & NPTX1 & {$[52]$} \\
\hline
\end{tabular}


Table 1 (continued)

\begin{tabular}{|c|c|c|c|c|}
\hline Protein & Abbreviation & UniProtKB & Gene & Ref. \\
\hline $\begin{array}{l}\text { Neuropeptide Y } \\
\text { (cleaved form pro-neuropeptide Y) }\end{array}$ & NPY_HUMAN & P01303 & NPY & {$[65]$} \\
\hline Neurosecretory protein VGF* & VGF_HUMAN & O15240 & VGF & {$[52]$} \\
\hline Neuroserpin* & NEUS_HUMAN & Q99574 & SERPINI1 & {$[65]$} \\
\hline Neurturin & NRTN_HUMAN & Q99748 & NRTN & {$[65]$} \\
\hline Neutrophil collagenase* & MMP8_HUMAN & P22894 & MMP8 & {$[52]$} \\
\hline $\begin{array}{l}\text { Neutrophil-activating peptide } 2 \text { (cleaved from Platelet basic } \\
\text { protein)* }\end{array}$ & CXCL7_HUMAN & P02775 & PPBP & {$[65]$} \\
\hline Non-receptor tyrosine-protein kinase TYK2* & TYK2_HUMAN & P29597 & TYK2 & {$[65]$} \\
\hline Nucleoside diphosphate kinase A & NDKA_HUMAN & P15531 & NME1 & {$[65]$} \\
\hline Orexin receptor type $1^{*}$ & OX1R_HUMAN & $\mathrm{O} 43613$ & HCRTR1 & {$[65]$} \\
\hline OX-2 membrane glycoprotein* & OX2G_HUMAN & P41217 & CD200 & {$[65]$} \\
\hline Pentraxin-related protein PTX3 & PTX3_HUMAN & P26022 & PTX3 & {$[59,60]$} \\
\hline Peptide YY & PYY_HUMAN & P10082 & PYY & {$[65]$} \\
\hline Periostin & POSTN_HUMAN & Q15063 & POSTN & {$[59]$} \\
\hline Phosphatidylinositol 3-kinase regulatory subunit beta* & P85B_HUMAN & O00459 & PIK3R2 & {$[52]$} \\
\hline Phosphoglycerate Kinase 1 & PGK1_HUMAN & P00558 & PGK1 & {$[12]$} \\
\hline Plakophilin-1 & PKP1_HUMAN & Q13835 & PKP1 & {$[59]$} \\
\hline Plasma protease $\mathrm{C} 1$ inhibitor* & IC1_HUMAN & P05155 & SERPING1 & {$[52]$} \\
\hline Platelet endothelial cell adhesion molecule* & PECA1_HUMAN & P16284 & PECAM1 & {$[61]$} \\
\hline Platelet glycoprotein $4^{*}$ & CD36_HUMAN & P16671 & CD36 & {$[65]$} \\
\hline Platelet-derived growth factor $\mathrm{D}^{*}$ & PDGFD_HUMAN & Q9GZP0 & PDGFD & {$[65]$} \\
\hline Platelet-derived growth factor receptor alpha* & PGFRA_HUMAN & P16234 & PDGFRA & {$[52,57,65]$} \\
\hline Platelet-derived growth factor receptor beta* & PGFRB_HUMAN & P09619 & PDGFRB & {$[52,57]$} \\
\hline Platelet-derived growth factor subunit B & PDGFB_HUMAN & P01127 & PDGFB & {$[57]$} \\
\hline Polyubiquitin-B* & UBB_HUMAN & P0CG47 & UBB & {$[52,65]$} \\
\hline PPARg2*** & - & - & PPARG & {$[52]$} \\
\hline Probetacellulin* & BTC_HUMAN & P35070 & BTC & {$[52,65]$} \\
\hline Pro-epidermal growth factor* & EGF_HUMAN & P01133 & EGF & {$[61]$} \\
\hline Progesterone receptor & PRGR_HUMAN & P06401 & PGR & {$[52]$} \\
\hline pro-Glucagon & GLUC_HUMAN & P01275 & GCG & {$[65]$} \\
\hline Progranulin & GRN_HUMAN & P28799 & GRN & {$[65]$} \\
\hline Proheparin-binding EGF-like growth factor* & HBEGF_HUMAN & Q99075 & HBEGF & {$[65]$} \\
\hline Prokineticin- $1 *$ & PROK1_HUMAN & P58294 & PROK1 & {$[65]$} \\
\hline ProSAAS & PCS1N_HUMAN & Q9UHG2 & PCSK1N & {$[52]$} \\
\hline Prostaglandin D2 receptor $2 *$ & PD2R2_HUMAN & Q9Y5Y4 & PTGDR2 & {$[65]$} \\
\hline Protein AMBP* & AMBP_HUMAN & $\mathrm{P} 02760$ & AMBP & {$[65]$} \\
\hline Protein FAM3B & FAM3B_HUMAN & P58499 & FAM3B & {$[52,65]$} \\
\hline Protein S100-A6 & S10A6_HUMAN & P06703 & S100A6 & {$[65]$} \\
\hline Protein S100-A8 & S10A8_HUMAN & P05109 & S100A8 & {$[52,65]$} \\
\hline Protein S100-A10 & S10AA_HUMAN & P60903 & S100A10 & {$[65]$} \\
\hline Protein S100-A12* & S10AC_HUMAN & P80511 & S100A12 & {$[65]$} \\
\hline Protein Wnt-5a & WNT5A_HUMAN & P41221 & WNT5A & {$[57]$} \\
\hline Protein Wnt-5b & WNT5B_HUMAN & Q9H1J7 & WNT5B & {$[57]$} \\
\hline Protein wntless homolog & WLS_HUMAN & Q5T9L3 & WLS & {$[57]$} \\
\hline Protocadherin Fat 1 & FAT1_HUMAN & Q14517 & FAT1 & {$[57]$} \\
\hline Protocadherin Fat 4 & FAT4_HUMAN & Q6V0I7 & FAT4 & {$[57]$} \\
\hline Protocadherin gamma-C3 & PCDGK_HUMAN & Q9UN70 & PCDHGC3 & {$[57]$} \\
\hline Protocadherin-7 & PCDH7_HUMAN & O60245 & PCDH7 & {$[57]$} \\
\hline Protocadherin-9 & PCDH9_HUMAN & Q9HC56 & PCDH9 & [57] \\
\hline
\end{tabular}


Table 1 (continued)

\begin{tabular}{|c|c|c|c|c|}
\hline Protein & Abbreviation & UniProtKB & Gene & Ref. \\
\hline Protocadherin-18 & PCD18_HUMAN & Q9HCL0 & PCDH18 & {$[57]$} \\
\hline Proto-oncogene tyrosine-protein kinase receptor Ret* & RET_HUMAN & P07949 & RET & {$[65]$} \\
\hline P-selectin & LYAM3_HUMAN & P16109 & SELP & {$[52]$} \\
\hline Ras-related protein R-Ras & RRAS_HUMAN & P10301 & RRAS & {$[57]$} \\
\hline Ras-related protein R-Ras2 & RRAS2_HUMAN & P62070 & RRAS2 & {$[57]$} \\
\hline Receptor tyrosine-protein kinase erbB-2* & ERBB2_HUMAN & P04626 & ERBB2 & {$[65]$} \\
\hline Receptor tyrosine-protein kinase erbB-4* & ERBB4_HUMAN & Q15303 & ERBB4 & {$[65]$} \\
\hline $\begin{array}{l}\text { Receptor-interacting serine/ } \\
\text { threonine-protein kinase } 1^{*}\end{array}$ & RIPK1_HUMAN & Q13546 & RIPK1 & {$[65]$} \\
\hline Receptor-type tyrosine-protein kinase FLT3* & FLT3_HUMAN & P36888 & FLT3 & {$[65]$} \\
\hline $\begin{array}{l}\text { Receptor-type tyrosine-protein } \\
\text { phosphatase delta* }\end{array}$ & PTPRD_HUMAN & P23468 & PTPRD & {$[52]$} \\
\hline Rho family-interacting cell polarization regulator 1 & RIPR1_HUMAN & Q6ZS17 & RIPOR1 & {$[57]$} \\
\hline Rho GTPase-activating protein 1 & RHG01_HUMAN & Q07960 & ARHGAP1 & {$[57]$} \\
\hline Rho guanine nucleotide exchange factor 1 & ARHG1_HUMAN & Q92888 & ARHGEF1 & {$[57]$} \\
\hline Rho guanine nucleotide exchange factor 7 & ARHG7_HUMAN & Q14155 & ARHGEF7 & {$[57]$} \\
\hline Rho-associated protein kinase $1 *$ & ROCK1_HUMAN & Q13464 & ROCK1 & {$[52,57]$} \\
\hline Rho-associated protein kinase 2 & ROCK2_HUMAN & O75116 & ROCK2 & {$[57]$} \\
\hline Rho-related GTP-binding protein RhoB & RHOB_HUMAN & P62745 & RHOB & {$[57]$} \\
\hline Rho-related GTP-binding protein RhoE & RND3_HUMAN & P61587 & RND3 & {$[57]$} \\
\hline Rho-related GTP-binding protein RhoG & RHOG_HUMAN & P84095 & RHOG & {$[57]$} \\
\hline Ribosomal oxygenase $2^{*}$ & RIOX2_HUMAN & Q8IUF8 & RIOX2 & {$[52]$} \\
\hline Scavenger receptor cysteine-rich type 1 protein $\mathrm{M} 130^{*}$ & C163A_HUMAN & Q86VB7 & CD163 & {$[52]$} \\
\hline Sclerostin* & SOST_HUMAN & Q9BQB4 & SOST & {$[65]$} \\
\hline Secreted frizzled-related protein $1^{*}$ & SFRP1_HUMAN & Q8N474 & SFRP1 & {$[65]$} \\
\hline Serum amyloid A-1 protein* & SAA1_HUMAN & P0DJI8 & SAA1 & {$[52]$} \\
\hline Secreted frizzled-related protein $3^{*}$ & SFRP3_HUMAN & Q92765 & FRZB & {$[65]$} \\
\hline Secreted frizzled-related protein $4^{*}$ & SFRP4_HUMAN & Q6FHJ7 & SFRP4 & {$[60]$} \\
\hline Serine/threonine-protein kinase MRCK alpha & $\begin{array}{l}\text { MRCKA } \\
\text { HUMAN }\end{array}$ & Q5VT25 & CDC42BPA & {$[57]$} \\
\hline Serine/threonine-protein kinase MRCK beta & MRCKB_HUMAN & Q9Y5S2 & CDC42BPB & {$[57]$} \\
\hline Serotransferrin & TRFE_HUMAN & P02787 & $\mathrm{TF}$ & {$[59]$} \\
\hline Sex hormone-binding globulin* & SHBG_HUMAN & P04278 & SHBG & {$[52]$} \\
\hline Sialic acid-binding Ig-like lectin $5^{*}$ & SIGL5_HUMAN & O15389 & SIGLEC5 & {$[65]$} \\
\hline Sialic acid-binding Ig-like lectin $9 *$ & SIGL9_HUMAN & Q9Y336 & SIGLEC9 & {$[52]$} \\
\hline $\begin{array}{l}\text { Signal peptide, CUB and EGF-like domain-containing } \\
\text { protein } 3\end{array}$ & SCUB3_HUMAN & Q8IX30 & SCUBE3 & {$[57]$} \\
\hline Signal transducer CD24* & CD24_HUMAN & P25063 & $\mathrm{CD} 24$ & {$[65]$} \\
\hline SLIT-ROBO Rho GTPase-activating protein 1 & SRGP1_HUMAN & Q7Z6B7 & SRGAP1 & {$[57]$} \\
\hline SLIT-ROBO Rho GTPase-activating protein 2 & SRGP2_HUMAN & O75044 & SRGAP2 & {$[57]$} \\
\hline $\begin{array}{l}\text { Solute carrier family } 2 \text {, facilitated glucose transporter } \\
\text { member } 1 *\end{array}$ & GTR1_HUMAN & P11166 & SLC2A1 & {$[52,65]$} \\
\hline $\begin{array}{l}\text { Solute carrier family } 2 \text {, facilitated glucose transporter } \\
\text { member } 2^{*}\end{array}$ & GTR2_HUMAN & P11168 & SLC2A2 & {$[52]$} \\
\hline $\begin{array}{l}\text { Solute carrier family } 2 \text {, facilitated glucose transporter } \\
\text { member } 3^{*}\end{array}$ & GTR3_HUMAN & P11169 & SLC2A3 & {$[65]$} \\
\hline $\begin{array}{l}\text { Solute carrier family } 2 \text {, facilitated glucose transporter } \\
\text { member } 5^{*}\end{array}$ & GTR5_HUMAN & P22732 & SLC2A5 & {$[52,65]$} \\
\hline Somatotropin* & SOMA_HUMAN & P01241 & GH1 & {$[52]$} \\
\hline Sonic hedgehog protein* & SHH_HUMAN & Q15465 & $\mathrm{SHH}$ & {$[52]$} \\
\hline SPARC & SPRC_HUMAN & P09486 & SPARC & {$[60]$} \\
\hline Sphingosine 1-phosphate receptor $1 *$ & S1PR1_HUMAN & P21453 & S1PR1 & {$[52,65]$} \\
\hline
\end{tabular}


Table 1 (continued)

\begin{tabular}{|c|c|c|c|c|}
\hline Protein & Abbreviation & UniProtKB & Gene & Ref. \\
\hline Stromal cell-derived factor $1 *$ & SDF1_HUMAN & P48061 & CXCL12 & {$[52]$} \\
\hline Stromelysin-2* & MMP10_HUMAN & P09238 & MMP10 & {$[65]$} \\
\hline Stromelysin-3 & MMP11_HUMAN & P24347 & MMP11 & {$[52,65]$} \\
\hline $\begin{array}{l}\text { SWI/SNF-related matrix-associated actin-dependent } \\
\text { regulator of chromatin subfamily E member } 1 *\end{array}$ & SMCE1_HUMAN & Q969G3 & SMARCE1 & {$[65]$} \\
\hline TGF-beta $5 * * *$ & - & - & TGFB5 & {$[52]$} \\
\hline TGF-beta receptor type- 2 & TGFR2_HUMAN & P37173 & TGFBR2 & [57] \\
\hline Thioredoxin-interacting protein* & TXNIP_HUMAN & Q9H3M7 & TXNIP & {$[52,65]$} \\
\hline Thrombopoietin & TPO_HUMAN & P40225 & THPO & {$[65]$} \\
\hline Thrombospondin-1 & TSP1_HUMAN & P07996 & THBS1 & {$[59,60]$} \\
\hline Thrombospondin-2 & TSP2_HUMAN & P35442 & THBS2 & {$[52]$} \\
\hline Thyroid peroxidase* & PERT_HUMAN & P07202 & ТРO & {$[52]$} \\
\hline Thyrotropin subunit beta* & TSHB_HUMAN & P01222 & TSHB & {$[52]$} \\
\hline T lymphocyte activation antigen CD80* & CD80_HUMAN & P33681 & CD80 & {$[60,65]$} \\
\hline Toll-like receptor $2 *$ & TLR2_HUMAN & O60603 & TLR2 & {$[65]$} \\
\hline Toll-like receptor $4 *$ & TLR4_HUMAN & O00206 & TLR4 & {$[65]$} \\
\hline TRA-1-60 and TRA-1-81*** & - & - & PODXL & {$[52]$} \\
\hline Transcription factor SOX-2* & SOX2_HUMAN & P48431 & SOX2 & {$[65]$} \\
\hline Transcription initiation factor TFIID subunit $4 *$ & TAF4_HUMAN & O00268 & TAF4 & {$[65]$} \\
\hline Transferrin receptor protein $1 *$ & TFR1_HUMAN & P02786 & TFRC & {$[52,65]$} \\
\hline $\begin{array}{l}\text { Transforming growth factor alpha* (cleaved from } \\
\text { Protransforming growth factor alpha) }\end{array}$ & TGFA_HUMAN & P01135 & TGFA & [61] \\
\hline Transforming growth factor beta receptor type $3 *$ & TGBR3_HUMAN & Q03167 & TGFBR3 & {$[65]$} \\
\hline $\begin{array}{l}\text { Transforming growth factor beta- } 1 \text { (cleaved from } \\
\text { Transforming growth factor beta-1 proprotein)* }\end{array}$ & TGFB1_HUMAN & P01137 & TGFB1 & {$[52,57,65]$} \\
\hline $\begin{array}{l}\text { Transforming growth factor beta- } 3 * \text { (cleaved form } \\
\text { Transforming growth factor beta-3 proprotein) }\end{array}$ & TGFB3_HUMAN & P10600 & TGFB3 & [61] \\
\hline Transforming growth factor-beta-induced protein ig-h3 & BGH3_HUMAN & Q15582 & TGFBI & {$[57,59]$} \\
\hline Transforming protein RhoA & RHOA_HUMAN & P61586 & RHOA & {$[57]$} \\
\hline $\begin{array}{l}\text { Transient receptor potential cation channel subfamily } \\
\text { M member 7* }\end{array}$ & TRPM7_HUMAN & Q96QT4 & TRPM7 & {$[65]$} \\
\hline Triggering receptor expressed on myeloid cells $1^{*}$ & TREM1_HUMAN & Q9NP99 & TREM1 & {$[65]$} \\
\hline Troponin C, slow skeletal and cardiac muscles* & TNNC1_HUMAN & P63316 & TNNC1 & [52] \\
\hline Tumor necrosis factor ligand superfamily member $10^{*}$ & TNF10_HUMAN & P50591 & TNFSF10 & {$[65]$} \\
\hline Tumor necrosis factor ligand superfamily member $11^{*}$ & TNF11_HUMAN & O14788 & TNFSF11 & {$[65]$} \\
\hline Tumor necrosis factor ligand superfamily member $13^{*}$ & TNF13_HUMAN & O75888 & TNFSF13 & {$[60]$} \\
\hline Tumor necrosis factor ligand superfamily member $15^{*}$ & TNF15_HUMAN & O95150 & TNFSF15 & {$[65]$} \\
\hline Tumor necrosis factor ligand superfamily member $4 *$ & TNFL4_HUMAN & P23510 & TNFSF4 & {$[65]$} \\
\hline Tumor necrosis factor ligand superfamily member $6^{*}$ & TNFL6_HUMAN & P48023 & FASLG & {$[65]$} \\
\hline Tumor necrosis factor ligand superfamily member $8^{*}$ & TNFL8_HUMAN & P32971 & TNFRSF8 & {$[52,65]$} \\
\hline Tumor necrosis factor receptor superfamily member $10 \mathrm{~A}^{*}$ & TR10A_HUMAN & O00220 & TNFRSF10A & {$[52]$} \\
\hline Tumor necrosis factor receptor superfamily member $10 \mathrm{~B}^{*}$ & TR10B_HUMAN & O14763 & TNFRSF10B & {$[52]$} \\
\hline Tumor necrosis factor receptor superfamily member $11 \mathrm{~B}^{*}$ & TR11B_HUMAN & O00300 & TNFRSF11B & {$[60]$} \\
\hline Tumor necrosis factor receptor superfamily member $13 \mathrm{~B} *$ & TR13B_HUMAN & O14836 & TNFRSF13B & {$[52]$} \\
\hline Tumor necrosis factor receptor superfamily member $13 C^{*}$ & TR13C_HUMAN & Q96RJ3 & TNFRSF13C & {$[52,60,65]$} \\
\hline Tumor necrosis factor receptor superfamily member $6 \mathrm{~B}^{*}$ & TNF6B_HUMAN & O95407 & TNFRSF6B & {$[65]$} \\
\hline Tumor necrosis factor receptor superfamily member $14^{*}$ & TNR14_HUMAN & Q92956 & TNFRSF14 & {$[65]$} \\
\hline Tumor necrosis factor receptor superfamily member $17^{*}$ & TNR17_HUMAN & Q02223 & TNFRSF17 & {$[65]$} \\
\hline Tumor necrosis factor receptor superfamily member $19^{*}$ & TNR19_HUMAN & Q9NS68 & TNFRSF19 & {$[65]$} \\
\hline Tumor necrosis factor receptor superfamily member $25^{*}$ & TNR25_HUMAN & Q93038 & TNFRSF25 & {$[52,65]$} \\
\hline Tumor necrosis factor receptor superfamily member $27^{*}$ & TNR27_HUMAN & Q9HAV5 & EDA2R & {$[52,65]$} \\
\hline
\end{tabular}


Table 1 (continued)

\begin{tabular}{|c|c|c|c|c|}
\hline Protein & Abbreviation & UniProtKB & Gene & Ref. \\
\hline $\begin{array}{l}\text { Tumor necrosis factor receptor type 1-associated } \\
\text { DEATH domain protein* }\end{array}$ & TRADD_HUMAN & Q15628 & TRADD & {$[52]$} \\
\hline Tumor necrosis factor* & TNFA_HUMAN & P01375 & $\mathrm{TNF}$ & {$[52]$} \\
\hline Tyrosine-protein kinase ABL1* & ABL1_HUMAN & P00519 & ABL1 & {$[52,65]$} \\
\hline Tyrosine-protein kinase BTK* & BTK_HUMAN & Q06187 & BTK & {$[52,65]$} \\
\hline Tyrosine-protein kinase Fer* & FER_HUMAN & P16591 & FER & {$[52,65]$} \\
\hline Tyrosine-protein kinase FRK* & FRK_HUMAN & P42685 & FRK & {$[52]$} \\
\hline Tyrosine-protein kinase Fyn* & FYN_HUMAN & P06241 & FYN & {$[52,65]$} \\
\hline Tyrosine-protein kinase $\mathrm{HCK}^{*}$ & HCK_HUMAN & P08631 & HCK & {$[52]$} \\
\hline Tyrosine-protein kinase ITK/TSK* & ITK_HUMAN & Q08881 & ITK & {$[52]$} \\
\hline Tyrosine-protein kinase Lck* & LCK_HUMAN & P06239 & LCK & {$[52]$} \\
\hline Tyrosine-protein kinase Lyn* & LYN_HUMAN & P07948 & LYN & {$[52]$} \\
\hline Tyrosine-protein kinase receptor Tie- $1^{*}$ & TIE1_HUMAN & P35590 & TIE1 & {$[61]$} \\
\hline Tyrosine-protein kinase receptor UFO* & UFO_HUMAN & P30530 & AXL & {$[60,65]$} \\
\hline Tyrosine-protein kinase Tec* & TEC_HUMAN & P42680 & TEC & {$[52]$} \\
\hline Tyrosine-protein kinase TXK* & TXK_HUMAN & P42681 & TXK & {$[52]$} \\
\hline Tyrosine-protein kinase ZAP-70* & ZAP70_HUMAN & P43403 & ZAP70 & {$[52]$} \\
\hline Urokinase plasminogen activator surface receptor* & UPAR_HUMAN & Q03405 & PLAUR & {$[57,61]$} \\
\hline Vascular endothelial growth factor A* & VEGFA_HUMAN & P15692 & VEGFA & {$[61,65]$} \\
\hline Vascular endothelial growth factor $C^{*}$ & VEGFC_HUMAN & P49767 & VEGFC & {$[65]$} \\
\hline Vascular endothelial growth factor $\mathrm{D}^{*}$ & VEGFD_HUMAN & O43915 & VEGFD & {$[61,65]$} \\
\hline Vascular endothelial growth factor receptor $1^{*}$ & VGFR1_HUMAN & P17948 & FLT1 & {$[52]$} \\
\hline Vascular endothelial growth factor receptor $2^{*}$ & VGFR2_HUMAN & P35968 & KDR & {$[61,65]$} \\
\hline Vascular endothelial growth factor receptor $3 *$ & VGFR3_HUMAN & P35916 & FLT4 & [61] \\
\hline Vinculin & VINC_HUMAN & P18206 & VCL & {$[57]$} \\
\hline Vitamin D-binding protein & VTDB_HUMAN & P02774 & GC & {$[59]$} \\
\hline Vitronectin & VTNC_HUMAN & P04004 & VTN & {$[57]$} \\
\hline $\begin{array}{l}\text { WAP, Kazal, immunoglobulin, Kunitz and NTR } \\
\text { domain-containing protein } 1^{*}\end{array}$ & WFKN1_HUMAN & Q96NZ8 & WFIKKN1 & {$[52]$} \\
\hline $\mathrm{X}$-linked interleukin-1 receptor accessory protein-like $2^{*}$ & IRPL2_HUMAN & Q9NP60 & IL1RAPL2 & {$[65]$} \\
\hline
\end{tabular}

*The referred article used alternative or short names

**The specific member of this family detected has been not described

***Name and gene referred by the article cited

reported in Table $2 \mathrm{~S}$. The biological processes in which a relatively large number of proteins are involved are: developmental process, signaling and cell communication, cell adhesion, immune system process, cellular component organization, response to stimulus, regulation of cellular process, apoptotic process, cellular protein metabolic process, viral process, regulation of molecular function, locomotion, and positive regulation of gene expression (Fig. 3).

The proteins detected in AT-MSC-EV cargo are involved in a great number of biological processes, but only a few of these processes utilise a large number of proteins. The biological processes in which the largest number of proteins take part are cell adhesion (in which $18 \%$ of proteins are involved) and specific child terms of signaling and cell communication $(28 \%$ signal transduction), regulation of cellular processes $(18 \%$ positive regulation of cell population proliferation), immune system process (17\% immune response) and developmental processes (17\% multicellular organism development) (Fig. 4).

\section{Therapeutic Approaches of AT-MSC-EV Proteins}

These results illustrate the role of AT-MSC-EVs in cell-cell communication [3-6], and the promising therapeutic effects observed in different research fields. Regarding the musculoskeletal system, AT-MSC-EVs have shown protective effects against cartilage degeneration, promotion of cell proliferation and migration of osteoarthritis chondrocytes, and antisenescence effects in osteoarthritis osteoblasts in vitro and in vivo $[66,78]$. They have also shown protective properties on muscle damage in an in vivo model of hindlimb 


\section{Top 20 GO molecular function terms of proteins}

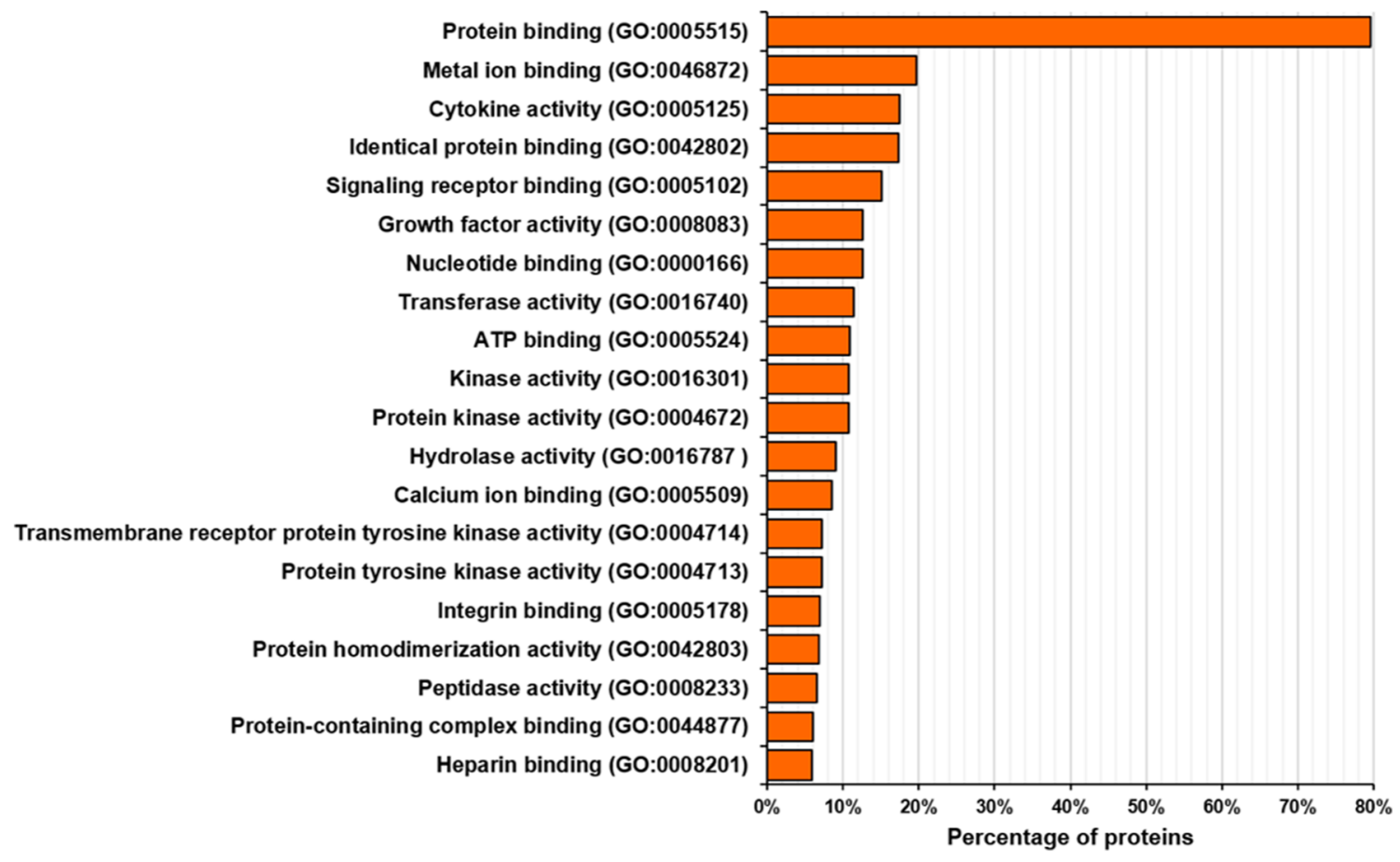

Fig. 1 The top 20 gene ontology (GO) molecular function terms of the proteins detected in human AT-MSC-EVs. The 80\% of the proteins associated with these EVs enables the protein binding

ischemia and in an in vitro model of ischemia/reperfusion [52]. These effects may be a consequence of the presence of proteins such as lactotransferrin, C-X-C motif chemokine 16, protein Wnt-5a, and transforming protein RhoA, which are involved in positive regulation of chondrocyte proliferation, positive regulation of cell migration, regulation of inflammatory response and regulation of osteoblast proliferation, respectively. The complete list of proteins involved in these processes is reported in Table $2 \mathrm{~S}$.

With regard to cardiology and vascular system, AT-MSCEVs are involved in a wide range of biological processes, including heart development, contraction and morphogenesis, positive regulation of cardiac muscle cell proliferation and hypertrophy, regulation of cardiac muscle cell apoptotic process and proliferation, blood vessel maturation, remodeling and morphogenesis, regulation of blood vessel diameter and angiogenesis, among others (Table 2S). Hence, numerous proteins detected in AT-MSC-EVs could account for the protective effects observed in cardiac function and cardiomyocytes after their injection in an in vivo model of myocardial infarction [79] . In addition, the effects of AT-MSC-EVs in angiogenesis have been also studied in vitro and in vivo [60, 72, 80]. Proteins detected in AT-MSC-EVs such as IL-1 alpha and apelin receptor are proangiogenic, while SPARC is antiangiogenic (Table 2S).

Human AT-MSC-EVs also have an inhibitory effect on vein graft neointima formation, as observed in a mouse model of vein grafting [81]. This effect correlated with decreased macrophage infiltration, attenuated inflammatory cytokine expression, and reduced activation of MAPK and phosphatidylinositol-3 kinase signaling pathways [81]. EV proteins potentially involved in these processes are thrombospondin-1 (inflammatory response), IL-4 (negative regulation of macrophage activation), growth factor receptor-bound protein 2 (regulation of MAPK cascade) and MAP kinase 1 (regulation of phosphatidylinositol 3-kinase signaling) (Table 2S).

The effects of AT-MSC-EVs proteins in the vascular system may also be related to the cardio-renal protection observed in a deoxycorticosterone acetate-salt hypertensive animal model [82]. Thus, the administration of AT-MSC-EVs in this in vivo model protected against renal damage, preserved renal function, reduced inflammatory response, prevented fibrosis in the kidney and in cardiac tissue, and conserved normal blood pressure [82]. The administration of AT-MSC-EVs also showed a renal protective effect in an in vivo model of acute kidney injury [83]. Proteins detected in AT-MSC-EVs such as integrin alpha-3, IL-4, IL-10, collagen alpha-2(I) chain or periostin could be implicated in these outcomes (Table 2S).

Finally, the action of AT-MSC-EVs in skin diseases has also been studied [62, 68, 84, 85]. Human AT-MSC-EVs enhanced cutaneous repair and regeneration, both in vitro and in vivo, by the promotion of cell migration and proliferation, the inhibition of cell apoptosis and the regulation of fibroblast differentiation during skin wound healing $[68,84$, 85]. This is unsurprising, considering that the main biological 
Fig. 2 Simplified outline of the main molecular functions enabled by proteins detected in EVs derived from human AT-MSC. For a complete review of the relationships between gene ontology terms see the chart view in the web-based tool QuickGO (https://www.ebi.ac.uk/ QuickGO/)

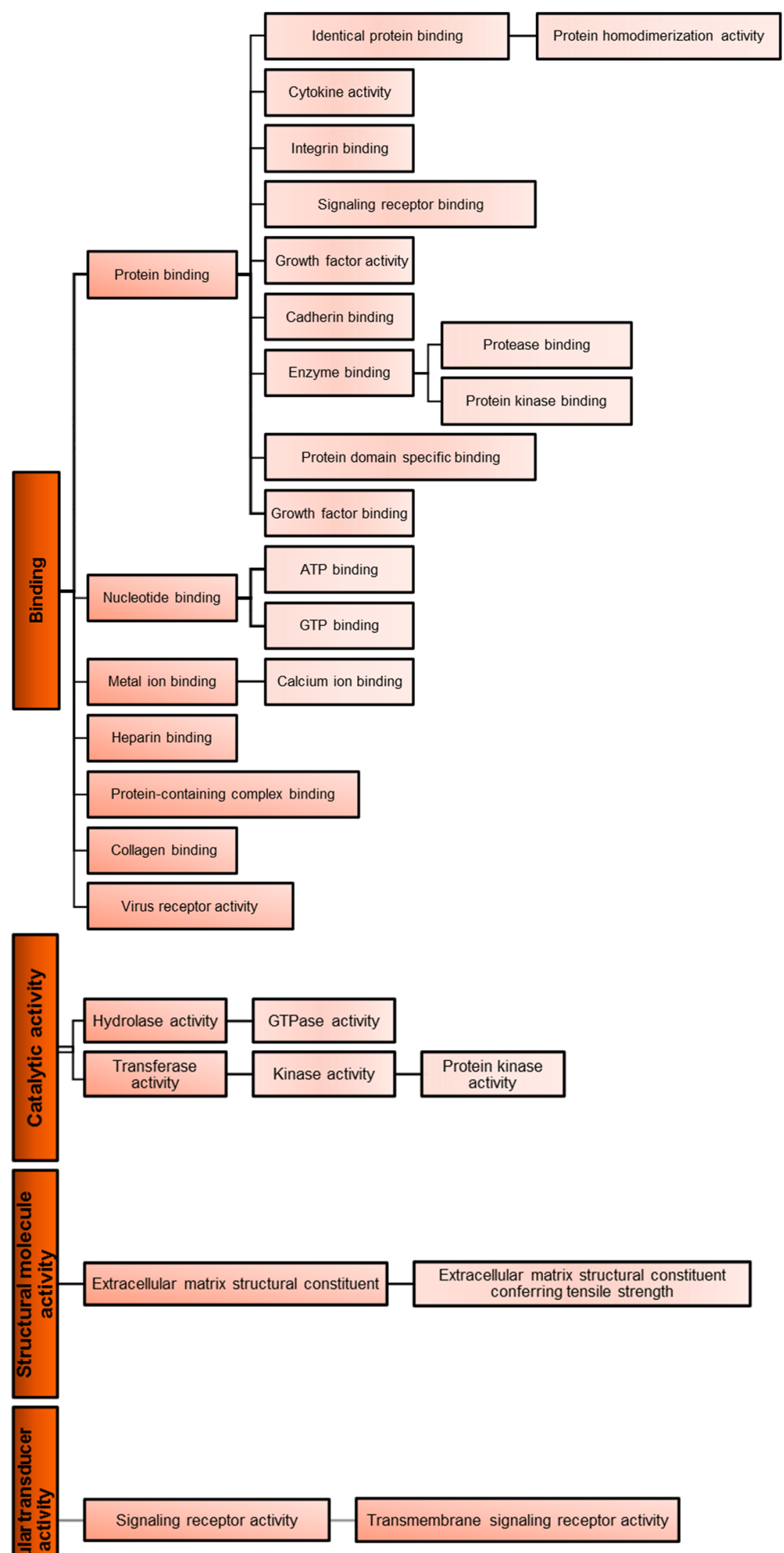



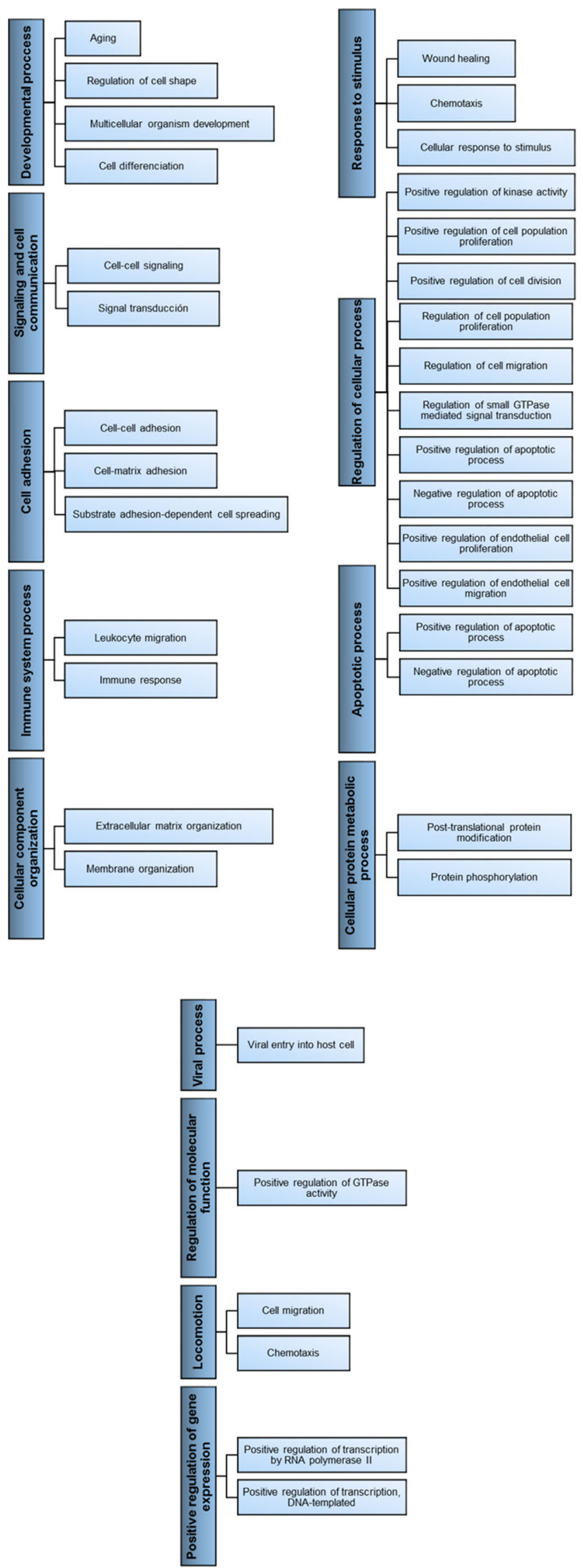

Fig. 3 Simplified outline of the main biological processes in which proteins detected in EVs derived from human AT-MSC are involved. For a complete review of the relationships between gene ontology terms see the chart view in the web-based tool QuickGO (https://www.ebi.ac. uk/QuickGO/)

processes of proteins described previously include response to stimulus (wound healing) and regulation of cellular processes (cell proliferation and migration) and apoptotic processes (Fig. 3, Table 2S). Proteins involved in these biological processes, along with those previously described in the vascular system, could support the protective effect of skin flaps against ischemia/reperfusion injury [62]. Although several proteins may be involved, in this study the observed effect was ascribed to the promotion of angiogenesis via IL-6, along with other mechanisms [62].

\section{miRNA}

AT-MSC-EVs cargo also contains several types of RNA, mainly miRNA, tRNA, mRNA, rRNA, snRNA, snoRNA and scRNA [53, 54]. AT-MSC-EVs are rich in miRNA [12, $54,69,70]$, which represents approximately $44 \%$ of all small, non-coding RNA detected in AT-MSC [53]. Currently, 604 miRNAs have been identified in AT-MSC-EVs (Table 2). The methods used for RNA analysis were sequencing systems [11, $53,54,59,66,67,71,74]$, quantitative real-time PCR [64, 65, $68,72,73]$, OpenArray systems $[69,70]$ and GeneChip RNA array [12], among others. The isolation methods of EVs used in those studies were centrifugation and/or ultracentrifugation $[12,64,65,67-69,72,74]$, commercial EV isolation kits [11, $53,54,59,71,73]$ and multi-filtration [66].

In this review, we present a comprehensive analysis of miRNAs currently identified in human AT-MSC-EVs. 489 miRNAs from 255 gene families were classified. The mir515 and mir-10 families have the greatest numbers of miRNAs (Table 2). However, there was no information available about which gene families the other 115 miRNAs belonged to. In addition, hsa-miR-320a-3p and hsa-miR$375-3 p$ were identified by the sequence and the precursor reported by Reza et al. [54], since the actual names used in the reference, hsa-miR-320a and hsa-miR-375, respectively, were not found for mature miRNA in any of the databases. Hsa-miR-1273a [54, 66] was included in the miRBase database as a dead miRNA entry. It was eventually removed due to lack of consistency between the patterns of mapped reads from RNA-sequencing experiments and the gene being processed as a miRNA. hsa-miR-1274a, hsa-miR-1274b, hsamiR-1300 and hsa-miR-720 [65] were also included in the miRBase database as dead miRNA entries. They were removed because it is likely that they are fragments of tRNAs and mRNA. This could be the reason for their absence from 


\section{Top 20 GO biological process terms of proteins}

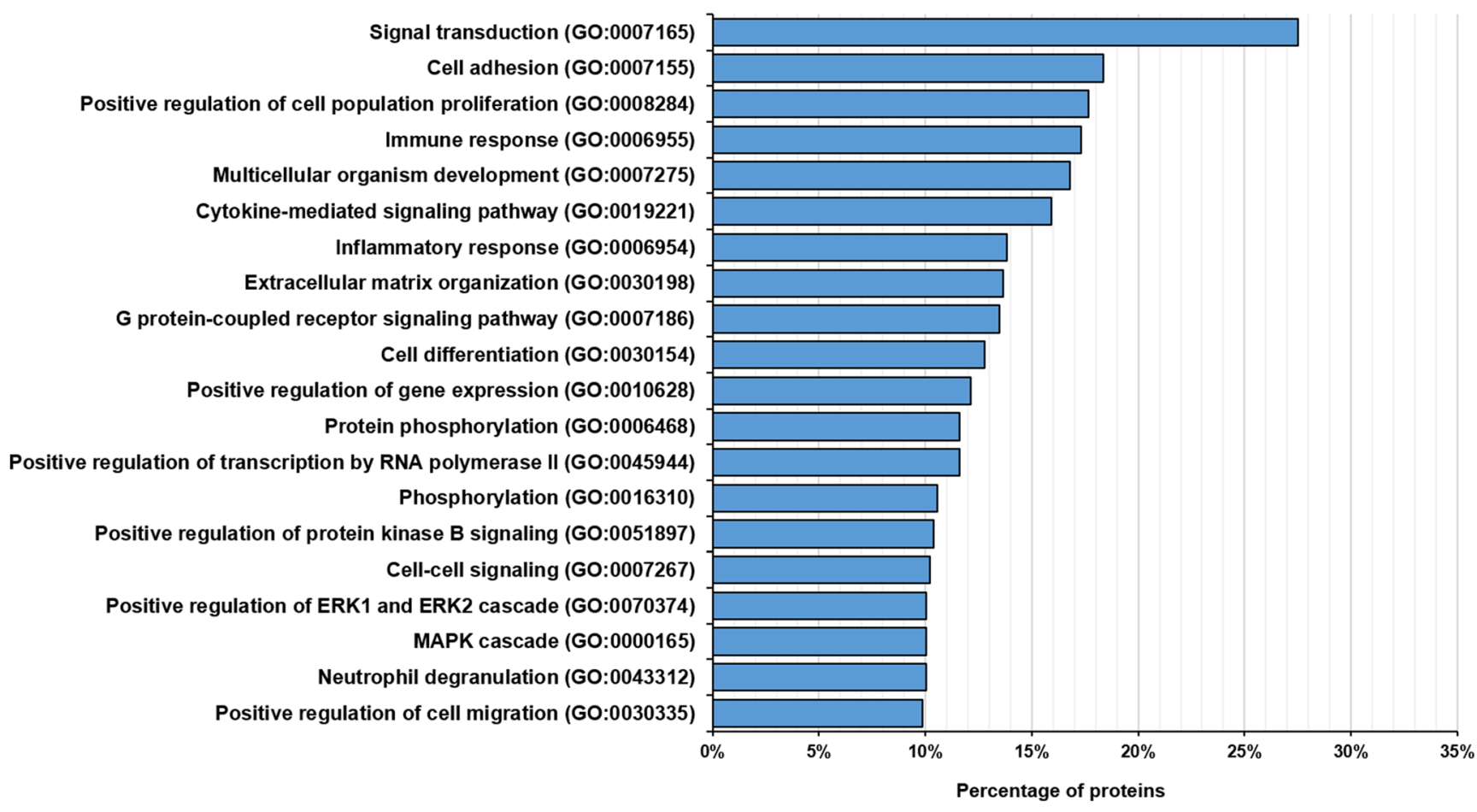

Fig. 4 The top 20 gene ontology (GO) biological process terms of the proteins detected in human AT-MSC-EVs. The $28 \%$ of the proteins are involved in signal transduction

the RNAcentral database. 44 miRNAs were not found in any of the databases (Table 2). Other special cases included hsamiR-548aa and hsa-miR-548 t-3p [66] - there is a specific entry for each one in the miRBase database, however, both entries showed the same sequence and RNAcentral link. Therefore, in the present review they are treated as the same miRNA. The same applies to hsa-miR-199b-3p and hsa-miR199a-3p [53, 65, 66, 72].

The variety of miRNAs present in AT-MSC-EVs may play a role in the different therapeutic effects based on the paracrine properties of MSC [13]. Regardless, to confirm the involvement of miRNAs in these effects, it is necessary to take into consideration not only the presence of a specific miRNA, but also other factors such as concentration, structure, and availability of accessory proteins [13].

Only 199 miRNA showed GO annotations for molecular function when using the QuickGO database [55]. The molecular functions enabled by these miRNAs are mRNA binding involved in post-transcriptional gene silencing (95\%), mRNA 3'-UTR binding (22\%), RNA polymerase II complex binding (6\%), single-stranded RNA binding and high-density lipoprotein particle binding ( $2 \%$ each), protein binding, transcription regulatory region sequence-specific DNA binding and sequence-specific single stranded DNA binding (1\% each) (Fig. 5). All of these functions are specific child terms of the binding function (Fig. 6) which is also the most relevant molecular function of AT-MSC-EV proteins, as previously described. The specific molecular functions enabled by each miRNA are detailed in Table $3 \mathrm{~S}$.

The number of miRNAs with GO annotations of biological processes in QuickGO [55] was 212. These miRNAs take part in biological processes described by 577 different GO terms. The biological processes in which the greatest number of miRNA are involved are: negative regulation of gene expression, response to stimulus, regulation of cellular process, developmental process, locomotion, signaling, and cell communication (Fig. 7). The specific miRNAs involved in each process are detailed in Table 4S. 89\% of these miRNAs are involved in gene silencing (Fig. 8). Other relevant GO terms in which a large number of miRNAs are included are miRNA mediated inhibition of translation (28\%) negative regulation of gene expression (17\%), negative regulation of angiogenesis (14\%), negative regulation of inflammatory response (13\%) and negative regulation of cell migration involved in sprouting angiogenesis (11\%) (Fig. 8).

\section{Therapeutic approaches of AT-MSC-EV miRNAs}

Based on the data, miRNAs present in AT-MSC-EV cargo support their potential use as new treatments in various research fields. Similar to proteins, different miRNAs are involved in inflammatory response (hsa-let-7 g-5p, hsa-miR16-5p, hsa-miR-92a-3p), negative regulation of macrophage activation (hsa-miR-124-3p), regulation of MAPK cascade 
Table 2 miRNAs detected in human AT-MSC-EVs in alphabetical order

\begin{tabular}{|c|c|c|c|c|}
\hline Family & Name & RNAcental & Sequence & Ref. \\
\hline \multirow{12}{*}{$\begin{array}{l}\text { let-7 } \\
{[74]}\end{array}$} & hsa-let-7a-3p & URS000004F5D8_9606 & CUAUACAAUCUACUGUCUUUC & {$[53]$} \\
\hline & $\begin{array}{l}\text { hsa-let-7a-5p } \\
\text { (hsa-let-7a) [65] }\end{array}$ & URS0000416056_9606 & UGAGGUAGUAGGUUGUAUAGUU & {$[11,12,53,54,65,66,69]$} \\
\hline & $\begin{array}{l}\text { hsa-let-7b-3p } \\
\text { (hsa-let-7b*) [65] }\end{array}$ & URS00005918D5_9606 & CUAUACAACCUACUGCCUUCCC & {$[53,65]$} \\
\hline & $\begin{array}{l}\text { hsa-let-7b-5p } \\
\text { (hsa-let-7b) [65] }\end{array}$ & URS0000324096_9606 & UGAGGUAGUAGGUUGUGUGGUU & {$[12,53,54,65,66]$} \\
\hline & hsa-let-7c-5p & URS000050DE77_9606 & UGAGGUAGUAGGUUGUAUGGUU & {$[12,53,54]$} \\
\hline & $\begin{array}{l}\text { hsa-let-7d-5p } \\
\text { (hsa-let-7d) [65] }\end{array}$ & URS00000A07C1_9606 & AGAGGUAGUAGGUUGCAUAGUU & {$[54,65]$} \\
\hline & $\begin{array}{l}\text { hsa-let-7e-5p } \\
\text { (hsa-let-7e) [65] }\end{array}$ & URS000000B1C9_9606 & UGAGGUAGGAGGUUGUAUAGUU & {$[12,53,54,65,66]$} \\
\hline & $\begin{array}{l}\text { hsa-let-7f-5p } \\
\text { (hsa-let-7f) }[65,67]\end{array}$ & URS00003B7674_9606 & UGAGGUAGUAGAUUGUAUAGUU & {$[11,53,54,65-67,72]$} \\
\hline & $\begin{array}{l}\text { hsa-let-7 g-5p } \\
\text { (hsa-let-7 g) [65] }\end{array}$ & URS00004AFF8D_9606 & UGAGGUAGUAGUUUGUACAGUU & {$[54,65]$} \\
\hline & $\begin{array}{l}\text { hsa-let-7i-3p } \\
\text { (hsa-let-7i*) }\end{array}$ & URS0000237CBD_9606 & CUGCGCAAGCUACUGCCUUGCU & {$[65]$} \\
\hline & hsa-let-7i-5p & URS00004023EA_9606 & UGAGGUAGUAGUUUGUGCUGUU & {$[53,54,72]$} \\
\hline & $\begin{array}{l}\text { hsa-miR-98-5p } \\
\text { (hsa-miR-98) [65] }\end{array}$ & URS00004E0808_9606 & UGAGGUAGUAAGUUGUAUUGUU & {$[53,65]$} \\
\hline mir-1 & hsa-miR-206 & URS000034B6F5_9606 & UGGAAUGUAAGGAAGUGUGUGG & {$[65]$} \\
\hline \multirow[t]{15}{*}{$\operatorname{mir}-10$} & $\begin{array}{l}\text { hsa-miR-100-3p } \\
\text { (hsa-miR-100*) }\end{array}$ & URS00001A405B_9606 & CAAGCUUGUAUCUAUAGGUAUG & {$[65]$} \\
\hline & $\begin{array}{l}\text { hsa-miR-100-5p } \\
\text { (hsa-miR-100) [65, 74] }\end{array}$ & URS000040D674_9606 & AACCCGUAGAUCCGAACUUGUG & {$[11,12,53,54,65,74]$} \\
\hline & $\begin{array}{l}\text { hsa-miR-10a-3p } \\
\text { (hsa-miR-10a*) }\end{array}$ & URS00002F4762_9606 & CAAAUUCGUAUCUAGGGGAAUA & {$[65]$} \\
\hline & $\begin{array}{l}\text { hsa-miR-10a-5p } \\
\text { (hsa-miR-10a) }[65,67]\end{array}$ & URS000016D2D4_9606 & UACCCUGUAGAUCCGAAUUUGUG & {$[11,53,54,65,67]$} \\
\hline & $\begin{array}{l}\text { hsa-miR-10b-3p } \\
\text { (hsa-miR-10b*) [65] }\end{array}$ & URS00004AC389_9606 & ACAGAUUCGAUUCUAGGGGAAU & {$[53,65,70]$} \\
\hline & $\begin{array}{l}\text { hsa-miR-10b-5p } \\
\quad \text { (hsa-miR-10b) }[65,67]\end{array}$ & URS000058760A_9606 & UACCCUGUAGAACCGAAUUUGUG & {$[11,53,54,65,67]$} \\
\hline & hsa-miR-125a-3p & URS00001F0C23_9606 & ACAGGUGAGGUUCUUGGGAGCC & {$[65]$} \\
\hline & hsa-miR-125a-5p & URS00005A4DCF_9606 & UCCCUGAGACCCUUUAACCUGUGA & {$[53,54,65]$} \\
\hline & $\begin{array}{l}\text { hsa-miR-125b-1-3 } \\
\text { (hsa-miR-125b-1*)[65]p }\end{array}$ & $\begin{array}{l}\text { URS00002DABEA_ } \\
9606\end{array}$ & ACGGGUUAGGCUCUUGGGAGCU & {$[53,54,65]$} \\
\hline & $\begin{array}{l}\text { hsa-miR-125b-2-3p } \\
\text { (hsa-miR-125b-2*) }\end{array}$ & URS00001925C1_9606 & UCACAAGUCAGGCUCUUGGGAC & {$[65]$} \\
\hline & $\begin{array}{l}\text { hsa-miR-125b-5p } \\
\text { (hsa-miR-125b) [65] }\end{array}$ & URS0000209905_9606 & UCCCUGAGACCCUAACUUGUGA & {$[12,53,54,65,66,72]$} \\
\hline & hsa-miR-99a-3p & URS00005C62FC_9606 & CAAGCUCGCUUCUAUGGGUCUG & {$[65]$} \\
\hline & hsa-miR-99a-5p & URS0000157026_9606 & AACCCGUAGAUCCGAUCUUGUG & {$[54,65]$} \\
\hline & $\begin{array}{l}\text { hsa-miR-99b-3p } \\
\text { (hsa-miR-99b*) }\end{array}$ & URS00001C308D_9606 & CAAGCUCGUGUCUGUGGGUCCG & {$[65]$} \\
\hline & $\begin{array}{l}\text { hsa-miR-99b-5p } \\
\text { (hsa-miR-99b) [65] }\end{array}$ & URS00002C10B3_9606 & CACCCGUAGAACCGACCUUGCG & {$[11,54,65]$} \\
\hline mir-101 & $\begin{array}{l}\text { hsa-miR-101-3p } \\
\quad \text { (hsa-miR-101) [65] }\end{array}$ & URS00001230A0_9606 & UACAGUACUGUGAUAACUGAA & {$[54,65,69]$} \\
\hline \multirow[t]{2}{*}{ mir-103 } & $\begin{array}{l}\text { hsa-miR-103a-3p } \\
\quad \text { (hsa-miR-103) [65] }\end{array}$ & URS0000476BE1_9606 & AGCAGCAUUGUACAGGGCUAUGA & {$[12,54,65,69]$} \\
\hline & hsa-miR-107 & URS00005743AE_9606 & AGCAGCAUUGUACAGGGCUAUCA & {$[54,65]$} \\
\hline mir-1179 & hsa-miR-1179 & URS000048B5E9_9606 & AAGCAUUCUUUCAUUGGUUGG & {$[65]$} \\
\hline mir-1183 & hsa-miR-1183 & URS000075A336_9606 & $\begin{array}{l}\text { CACUGUAGGUGAUGGUGAGA } \\
\text { GUGGGCA }\end{array}$ & {$[65]$} \\
\hline mir-1204 & hsa-miR-1204 & URS000075E520_9606 & UCGUGGCCUGGUCUCCAUUAU & {$[65]$} \\
\hline mir-1207 & hsa-miR-1207-5p & URS000055C019_9606 & UGGCAGGGAGGCUGGGAGGGG & {$[72]$} \\
\hline $\operatorname{mir}-1208$ & hsa-miR-1208 & URS000075B904_9606 & UCACUGUUCAGACAGGCGGA & {$[65]$} \\
\hline
\end{tabular}


Table 2 (continued)

\begin{tabular}{|c|c|c|c|c|}
\hline Family & Name & RNAcental & Sequence & Ref. \\
\hline $\operatorname{mir}-122$ & $\begin{array}{l}\text { hsa-miR-122-5p } \\
\text { (hsa-miR-122) [65] }\end{array}$ & URS00003380CC_9606 & UGGAGUGUGACAAUGGUGUUUG & {$[59,65]$} \\
\hline \multirow[t]{2}{*}{ mir-1225 } & hsa-miR-1225-3p & URS000075D62D_9606 & UGAGCCCCUGUGCCGCCCCCAG & {$[65]$} \\
\hline & hsa-miR-1225-5p & URS000075D0F5_9606 & GUGGGUACGGCCCAGUGGGGGG & [72] \\
\hline mir-1226 & hsa-miR-1226-5p & URS000075EAB0_9606 & GUGAGGGCAUGCAGGCCUGGAUGGGG & {$[65]$} \\
\hline $\operatorname{mir}-1227$ & $\begin{array}{l}\text { hsa-miR-1227-3p } \\
\text { (hsa-miR-1227) }\end{array}$ & URS000075CFA8_9606 & CGUGCCACCCUUUUCCCCAG & {$[65]$} \\
\hline mir-1228 & $\begin{array}{l}\text { hsa-miR-1228-5p } \\
\text { (hsa-miR-1228*) [65] }\end{array}$ & URS00004F1E01_9606 & GUGGGCGGGGGCAGGUGUGUG & {$[65,67]$} \\
\hline mir-1233 & $\begin{array}{l}\text { hsa-miR-1233-3p } \\
\text { (hsa-miR-1233) }\end{array}$ & URS000075D36A_9606 & UGAGCCCUGUCCUCCCGCAG & {$[65]$} \\
\hline mir-1238 & $\begin{array}{l}\text { hsa-miR-1238-3p } \\
\text { (hsa-miR-1238) }\end{array}$ & URS000075E57E_9606 & CUUCCUCGUCUGUCUGCCCC & {$[65]$} \\
\hline $\operatorname{mir}-124$ & hsa-miR-124-3p & URS000020BE6A_9606 & UAAGGCACGCGGUGAAUGCC & {$[54]$} \\
\hline mir-1244 & hsa-miR-1244 & URS000075B58F_9606 & AAGUAGUUGGUUUGUAUGAGAUGGUU & {$[65]$} \\
\hline mir-1246 & hsa-miR-1246 & URS000028C188_9606 & AAUGGAUUUUUGGAGCAGG & {$[54,66,72]$} \\
\hline \multirow[t]{2}{*}{ mir-1247 } & hsa-miR-1247-3p & URS000032835F_9606 & CCCCGGGAACGUCGAGACUGGAGC & {$[67]$} \\
\hline & $\begin{array}{l}\text { hsa-miR-1247-5p } \\
\text { (hsa-miR-1247) [65] }\end{array}$ & URS000057DF36_9606 & ACCCGUCCCGUUCGUCCCCGGA & {$[65,67]$} \\
\hline mir-1249 & $\begin{array}{l}\text { hsa-miR-1249-3p } \\
\text { (hsa-miR-1249) }\end{array}$ & $\begin{array}{l}\text { URS000060AABB_ } \\
\quad 9606\end{array}$ & ACGCCCUUCCCCCCCUUCUUCA & {$[65]$} \\
\hline mir-1253 & hsa-miR-1253 & URS000075A7EC_9606 & AGAGAAGAAGAUCAGCCUGCA & {$[65]$} \\
\hline mir-1254 & hsa-miR-1254 & URS000047047A_9606 & AGCCUGGAAGCUGGAGCCUGCAGU & {$[54,64]$} \\
\hline mir-1255 & $\begin{array}{l}\text { hsa-miR-1255b-5p } \\
\text { (hsa-miR-1255b) }\end{array}$ & URS0000211070_9606 & CGGAUGAGCAAAGAAAGUGGUU & {$[65]$} \\
\hline $\operatorname{mir}-1256$ & hsa-miR-1256 & URS0000098B3B_9606 & AGGCAUUGACUUCUCACUAGCU & {$[65]$} \\
\hline \multirow[t]{2}{*}{$\operatorname{mir}-126$} & $\begin{array}{l}\text { hsa-miR-126-3p } \\
\text { (hsa-miR-126) [65] }\end{array}$ & URS00001F1DA8_9606 & UCGUACCGUGAGUAAUAAUGCG & {$[54,65]$} \\
\hline & $\begin{array}{l}\text { hsa-miR-126-5p } \\
\text { (hsa-miR-126*) [65] }\end{array}$ & URS00001D69F6_9606 & CAUUAUUACUUUUGGUACGCG & {$[54,65]$} \\
\hline $\operatorname{mir}-1260 \mathrm{a}$ & $\begin{array}{l}\text { hsa-miR-1260a } \\
\text { (hsa-miR-1260) }\end{array}$ & URS00000D0874_9606 & AUCCCACCUCUGCCACCA & {$[65]$} \\
\hline $\operatorname{mir}-1260 b$ & hsa-miR-1260b & URS0000239117_9606 & AUCCCACCACUGCCACCAU & {$[12]$} \\
\hline mir-1262 & hsa-miR-1262 & URS0000568FF8_9606 & AUGGGUGAAUUUGUAGAAGGAU & {$[65]$} \\
\hline $\operatorname{mir}-1267$ & hsa-miR-1267 & URS000075AEB2_9606 & CCUGUUGAAGUGUAAUCCCCA & {$[65]$} \\
\hline $\operatorname{mir}-1268$ & hsa-miR-1268a & URS00005A8A8D_9606 & CGGGCGUGGUGGUGGGGG & [72] \\
\hline $\operatorname{mir}-127$ & $\begin{array}{l}\text { hsa-miR-127-3p } \\
\text { (hsa-miR-127) [65] }\end{array}$ & $\begin{array}{l}\text { URS00001E3DAA_ } \\
9606\end{array}$ & UCGGAUCCGUCUGAGCUUGGCU & {$[11,54,65,71]$} \\
\hline $\operatorname{mir}-1270$ & hsa-miR-1270 & URS00002E0524_9606 & CUGGAGAUAUGGAAGAGCUGUGU & {$[65]$} \\
\hline mir-1271 & $\begin{array}{l}\text { hsa-miR-1271-5p } \\
\text { (hsa-miR-1271) }\end{array}$ & URS00001F61BA_9606 & CUUGGCACCUAGCAAGCACUCA & {$[65]$} \\
\hline mir-1272 & hsa-miR-1272 & URS00000E1E9E_9606 & GAUGAUGAUGGCAGCAAAUUCUGAAA & {$[65]$} \\
\hline \multirow[t]{5}{*}{$\operatorname{mir}-1273$} & hsa-miR-1273a & - & GGGCGACAAAGCAAGACUCUUUCUU & {$[54,66]$} \\
\hline & hsa-miR-1273d & URS00003CF845_9606 & GAACCCAUGAGGUUGAGGCUGCAGU & {$[54]$} \\
\hline & hsa-miR-1273e & URS0000361F30_9606 & UUGCUUGAACCCAGGAAGUGGA & [54] \\
\hline & hsa-miR-1273f & URS00003DD70F_9606 & GGAGAUGGAGGUUGCAGUG & {$[54,66]$} \\
\hline & hsa-miR-1273g-3p & URS00002B60FB_9606 & ACCACUGCACUCCAGCCUGAG & {$[12,54,66]$} \\
\hline $\operatorname{miR}-1275$ & hsa-miR-1275 & URS000009EA8F_9606 & GUGGGGGAGAGGCUGUC & {$[65,73]$} \\
\hline \multirow[t]{2}{*}{$\operatorname{mir}-128$} & hsa-miR-128-1-5p & URS0000537082_9606 & CGGGGCCGUAGCACUGUCUGAGA & {$[67]$} \\
\hline & $\begin{array}{l}\text { hsa-miR-128-3p } \\
\text { (hsa-miR-128a) [65] }\end{array}$ & URS000024A59E_9606 & UCACAGUGAACCGGUCUCUUU & {$[54,65]$} \\
\hline \multirow[t]{2}{*}{$\operatorname{mir}-1285$} & $\begin{array}{l}\text { hsa-miR-1285-3p } \\
\text { (hsa-miR-1285) [65] }\end{array}$ & URS0000399545_9606 & UCUGGGCAACAAAGUGAGACCU & {$[65,66]$} \\
\hline & hsa-miR-1285-5p & URS000050A3A3_9606 & GAUCUCACUUUGUUGCCCAGG & {$[54,66]$} \\
\hline mir-129 & hsa-miR-129-2-3p & URS000048F59D_9606 & AAGCCCUUACCCCAAAAAGCAU & {$[54]$} \\
\hline
\end{tabular}


Table 2 (continued)

\begin{tabular}{|c|c|c|c|c|}
\hline Family & Name & RNAcental & Sequence & Ref. \\
\hline & hsa-miR-129-5p & URS00004E1410_9606 & CUUUUUGCGGUCUGGGCUUGC & {$[54]$} \\
\hline $\operatorname{mir}-1290$ & hsa-miR-1290 & URS000043F369_9606 & UGGAUUUUUGGAUCAGGGA & {$[54,65,66]$} \\
\hline $\operatorname{mir}-1291$ & hsa-miR-1291 & URS000047E28E_9606 & UGGCCCUGACUGAAGACCAGCAGU & {$[54,65]$} \\
\hline $\operatorname{mir}-1292$ & hsa-miR-1292-5p & URS00005586D0_9606 & UGGGAACGGGUUCCGGCAGACGCUG & {$[67]$} \\
\hline \multirow[t]{5}{*}{$\operatorname{mir}-130$} & $\begin{array}{l}\text { hsa-miR-130a-3p } \\
\text { (hsa-miR-130a) }\end{array}$ & URS0000315338_9606 & CAGUGCAAUGUUAAAAGGGCAU & {$[65]$} \\
\hline & $\begin{array}{l}\text { hsa-miR-130b-5p } \\
\text { (hsa-miR-130b*) }\end{array}$ & URS000032A4F7_9606 & ACUCUUUCCCUGUUGCACUAC & {$[65]$} \\
\hline & $\begin{array}{l}\text { hsa-miR-130b-3p } \\
\text { (hsa-miR-130b) }\end{array}$ & URS00002C0FCB_9606 & CAGUGCAAUGAUGAAAGGGCAU & {$[65]$} \\
\hline & $\begin{array}{l}\text { hsa-miR-301a-3p } \\
\text { (hsa-miR-301) }\end{array}$ & URS00001C11BC_9606 & CAGUGCAAUAGUAUUGUCAAAGC & {$[65]$} \\
\hline & $\begin{array}{l}\text { hsa-miR-301b-3p } \\
\text { (hsa-miR-301b) }\end{array}$ & URS0000251D0B_9606 & CAGUGCAAUGAUAUUGUCAAAGC & {$[65]$} \\
\hline mir-1303 & hsa-miR-1303 & URS000032FC1A_9606 & UUUAGAGACGGGGUCUUGCUCU & {$[54,65]$} \\
\hline $\operatorname{mir}-1305$ & hsa-miR-1305 & URS000040EC3B_9606 & UUUUCAACUCUAAUGGGAGAGA & {$[65]$} \\
\hline mir-1306 & hsa-miR-1306-5p & URS0000500449_9606 & CCACCUCCCCUGCAAACGUCCA & {$[67]$} \\
\hline mir-1307 & hsa-miR-1307-5p & URS00000EEF5F_9606 & UCGACCGGACCUCGACCGGCU & {$[54]$} \\
\hline \multirow[t]{3}{*}{ mir-132 } & $\begin{array}{l}\text { hsa-miR-132-3p } \\
\text { (hsa-miR-132) }\end{array}$ & URS00006054DA_9606 & UAACAGUCUACAGCCAUGGUCG & {$[65]$} \\
\hline & $\begin{array}{l}\text { hsa-miR-212-3p } \\
\text { (hsa-miR-212) }\end{array}$ & $\begin{array}{l}\text { URS00001D6BAE_ } \\
\quad 9606\end{array}$ & UAACAGUCUCCAGUCACGGCC & {$[65]$} \\
\hline & hsa-miR-212-5p & URS00001AFC71_9606 & ACCUUGGCUCUAGACUGCUUACU & {$[54]$} \\
\hline $\operatorname{mir}-134$ & hsa-miR-134-5p & URS0000272A92_9606 & UGUGACUGGUUGACCAGAGGGG & {$[54,59]$} \\
\hline mir-1343 & hsa-miR-1343-5p & URS0000759B67_9606 & UGGGGAGCGGCCCCCGGGUGGG & {$[67]$} \\
\hline \multirow[t]{2}{*}{ mir-135 } & $\begin{array}{l}\text { hsa-miR-135b-3p } \\
\text { (hsa-miR-135b*) }\end{array}$ & URS0000488C83_9606 & AUGUAGGGCUAAAAGCCAUGGG & {$[65]$} \\
\hline & $\begin{array}{l}\text { hsa-miR-135b-5p } \\
\text { (hsa-miR-135b) [65] }\end{array}$ & URS000001C659_9606 & UAUGGCUUUUCAUUCCUAUGUGA & {$[65,71]$} \\
\hline \multirow[t]{2}{*}{ mir-136 } & $\begin{array}{l}\text { hsa-miR-136-3p } \\
\text { (hsa-miR-136*) [65] }\end{array}$ & URS0000204177_9606 & CAUCAUCGUCUCAAAUGAGUCU & {$[54,65]$} \\
\hline & $\begin{array}{l}\text { hsa-miR-136-5p } \\
\text { (hsa-miR-136) [65] }\end{array}$ & URS00004EAB18_9606 & ACUCCAUUUGUUUUGAUGAUGGA & {$[54,65]$} \\
\hline \multirow[t]{2}{*}{ mir-138 } & $\begin{array}{l}\text { hsa-miR-138-2-3p } \\
\text { (hsa-miR-138-2*) }\end{array}$ & URS000075AA94_9606 & GCUAUUUCACGACACCAGGGUU & {$[65]$} \\
\hline & $\begin{array}{l}\text { hsa-miR-138-5p } \\
\text { (hsa-miR-138) [65] }\end{array}$ & URS000040780F_9606 & AGCUGGUGUUGUGAAUCAGGCCG & {$[54,65]$} \\
\hline \multirow[t]{2}{*}{ mir-139 } & hsa-miR-139-3p & URS000023BE29_9606 & UGGAGACGCGGCCCUGUUGGAGU & {$[65]$} \\
\hline & hsa-miR-139-5p & URS000025D232_9606 & UCUACAGUGCACGUGUCUCCAGU & {$[54,65]$} \\
\hline $\operatorname{mir}-140$ & hsa-miR-140-3p & URS00000821E0_9606 & UACCACAGGGUAGAACCACGG & {$[54,65]$} \\
\hline \multirow[t]{2}{*}{ mir-142 } & hsa-miR-142-3p & URS00002620A7_9606 & UGUAGUGUUUCCUACUUUAUGGA & {$[65]$} \\
\hline & hsa-miR-142-5p & URS00001E0AEA_9606 & CAUAAAGUAGAAAGCACUACU & {$[65]$} \\
\hline mir-143 & $\begin{array}{l}\text { hsa-miR-143-3p } \\
\text { (hsa-miR-143) [65] }\end{array}$ & URS00005C2A6D_9606 & UGAGAUGAAGCACUGUAGCUC & {$[11,53,54,65]$} \\
\hline \multirow[t]{2}{*}{ mir-144 } & $\begin{array}{l}\text { hsa-miR-144-3p } \\
\text { (hsa-miR-144) [65] }\end{array}$ & URS000037C5A8_9606 & UACAGUAUAGAUGAUGUACU & {$[53,54,65]$} \\
\hline & $\begin{array}{l}\text { hsa-miR-144-5p } \\
\text { (hsa-miR-144*) }\end{array}$ & URS00002E92A8_9606 & GGAUAUCAUCAUAUACUGUAAG & {$[65]$} \\
\hline \multirow[t]{2}{*}{ mir-145 } & $\begin{array}{l}\text { hsa-miR-145-3p } \\
\text { (hsa-miR-145*) }\end{array}$ & URS000052F380_9606 & GGAUUCCUGGAAAUACUGUUCU & {$[65]$} \\
\hline & $\begin{array}{l}\text { hsa-miR-145-5p } \\
\text { (hsa-miR-145) [65] }\end{array}$ & URS0000527F89_9606 & GUCCAGUUUUCCCAGGAAUCCCU & {$[12,65,66]$} \\
\hline \multirow[t]{3}{*}{ mir-146 } & $\begin{array}{l}\text { hsa-miR-146a-5p } \\
\text { (hsa-miR-146a) [65] }\end{array}$ & URS000050B527_9606 & UGAGAACUGAAUUCCAUGGGUU & {$[11,65,69-71]$} \\
\hline & hsa-miR-146b-3p & URS000050CCE0_9606 & UGCCCUGUGGACUCAGUUCUGG & {$[65]$} \\
\hline & $\begin{array}{l}\text { hsa-miR-146b-5p } \\
\text { (hsa-miR-146b) [65] }\end{array}$ & URS000061B694_9606 & UGAGAACUGAAUUCCAUAGGCU & {$[11,65]$} \\
\hline
\end{tabular}


Table 2 (continued)

\begin{tabular}{|c|c|c|c|c|}
\hline Family & Name & RNAcental & Sequence & Ref. \\
\hline mir-1468 & hsa-miR-1468-5p & URS00002ECEE4_9606 & CUCCGUUUGCCUGUUUCGCUG & {$[54]$} \\
\hline \multirow[t]{5}{*}{ mir-148 } & $\begin{array}{c}\text { hsa-miR-148a-3p } \\
\text { (hsa-miR-148a) } \\
{[63,64,70]}\end{array}$ & URS00003BBF48_9606 & UCAGUGCACUACAGAACUUUGU & {$[54,64,65,74]$} \\
\hline & $\begin{array}{l}\text { hsa-miR-148a-5p } \\
\text { (hsa-miR-148a*) }\end{array}$ & URS00003E16E5_9606 & AAAGUUCUGAGACACUCCGACU & {$[65]$} \\
\hline & $\begin{array}{l}\text { hsa-miR-148b-3p } \\
\text { (hsa-miR-148b) }\end{array}$ & URS0000521626_9606 & UCAGUGCAUCACAGAACUUUGU & {$[65]$} \\
\hline & $\begin{array}{l}\text { hsa-miR-148b-5p } \\
\text { (hsa-miR-148b*) }\end{array}$ & URS00005A7A84_9606 & AAGUUCUGUUAUACACUCAGGC & {$[65]$} \\
\hline & hsa-miR-152-3p & URS00003AFD9B_9606 & UCAGUGCAUGACAGAACUUGG & {$[53,59]$} \\
\hline \multirow[t]{2}{*}{ mir-149 } & hsa-miR-149-3p & URS000042C6A6_9606 & AGGGAGGGACGGGGGCUGUGC & {$[12,67]$} \\
\hline & $\begin{array}{l}\text { hsa-miR-149-5p } \\
\text { (hsa-miR-149) }\end{array}$ & URS00001C770D_9606 & UCUGGCUCCGUGUCUUCACUCCC & {$[65]$} \\
\hline \multirow[t]{9}{*}{$\operatorname{mir}-15$} & $\begin{array}{l}\text { hsa-miR-15a-3p } \\
\text { (hsa-miR-15a*) }\end{array}$ & URS00001C94E0_9606 & CAGGCCAUAUUGUGCUGCCUCA & {$[65]$} \\
\hline & $\begin{array}{l}\text { hsa-miR-15a-5p } \\
\text { (hsa-miR-15a) }\end{array}$ & URS00003D1AE3_9606 & UAGCAGCACAUAAUGGUUUGUG & {$[65]$} \\
\hline & $\begin{array}{l}\text { hsa-miR-15b-3p } \\
\text { (hsa-miR-15b*) }\end{array}$ & URS000045A9D7_9606 & CGAAUCAUUAUUUGCUGCUCUA & {$[65]$} \\
\hline & $\begin{array}{l}\text { hsa-miR-15b-5p } \\
\text { (hsa-miR-15b) [65] }\end{array}$ & URS00004AD914_9606 & UAGCAGCACAUCAUGGUUUACA & {$[65,72]$} \\
\hline & $\begin{array}{l}\text { hsa-miR-16-1-3p } \\
\text { (hsa-miR-16-1*) }\end{array}$ & URS000061CB8F_9606 & CCAGUAUUAACUGUGCUGCUGA & {$[65]$} \\
\hline & $\begin{array}{l}\text { hsa-miR-16-2-3p } \\
\text { (hsa-miR-16-2*) }\end{array}$ & URS00001E9CCB_9606 & CCAAUAUUACUGUGCUGCUUUA & {$[65]$} \\
\hline & $\begin{array}{l}\text { hsa-miR-16-5p } \\
\text { (hsa-miR-16) [65] }\end{array}$ & $\begin{array}{l}\text { URS00004BCD9C_ } \\
\quad 9606\end{array}$ & UAGCAGCACGUAAAUAUUGGCG & {$[12,54,59,65,69]$} \\
\hline & hsa-miR-195-3p & URS0000476C64_9606 & CCAAUAUUGGCUGUGCUGCUCC & {$[53]$} \\
\hline & $\begin{array}{l}\text { hsa-miR-195-5p } \\
\text { (hsa-miR-195) }\end{array}$ & URS00005B3525_9606 & UAGCAGCACAGAAAUAUUGGC & {$[65]$} \\
\hline $\operatorname{mir}-150$ & $\begin{array}{l}\text { hsa-miR-150-5p } \\
\text { (hsa-miR-150) }\end{array}$ & URS000016FD1A_9606 & UCUCCCAACCCUUGUACCAGUG & {$[65]$} \\
\hline mir-153 & hsa-miR-153-3p & URS0000068B85_9606 & UUGCAUAGUCACAAAAGUGAUC & {$[54]$} \\
\hline mir-1538 & hsa-miR-1538 & URS00005235AA_9606 & CGGCCCGGGCUGCUGCUGUUCCU & {$[67]$} \\
\hline \multirow[t]{12}{*}{ mir-154 } & $\begin{array}{l}\text { hsa-miR-154-3p } \\
\text { (hsa-miR-154*) }\end{array}$ & URS00000C0921_9606 & AAUCAUACACGGUUGACCUAUU & {$[65]$} \\
\hline & $\begin{array}{l}\text { hsa-miR-323a-3p } \\
\text { (hsa-miR-323-3p) }\end{array}$ & $\begin{array}{l}\text { URS00003CCAB4_ } \\
\quad 9606\end{array}$ & CACAUUACACGGUCGACCUCU & {$[65]$} \\
\hline & $\begin{array}{l}\text { hsa-miR-323b-5p } \\
\text { (hsa-miR-453) }\end{array}$ & URS000075D04C_9606 & AGGUUGUCCGUGGUGAGUUCGCA & {$[65]$} \\
\hline & hsa-miR-369-3p & URS0000442B0D_9606 & AAUAAUACAUGGUUGAUCUUU & {$[65]$} \\
\hline & hsa-miR-369-5p & URS00002A71AD_9606 & AGAUCGACCGUGUUAUAUUCGC & {$[65]$} \\
\hline & $\begin{array}{l}\text { hsa-miR-377-5p } \\
\text { (hsa-miR-377*) }\end{array}$ & URS000036BEF1_9606 & AGAGGUUGCCCUUGGUGAAUUC & {$[65]$} \\
\hline & hsa-miR-381-3p & URS00001FFA8C_9606 & UAUACAAGGGCAAGCUCUCUGU & {$[54]$} \\
\hline & $\begin{array}{l}\text { hsa-miR-382-5p } \\
\text { (hsa-miR-382) }\end{array}$ & URS000035E174_9606 & GAAGUUGUUCGUGGUGGAUUCG & {$[65]$} \\
\hline & hsa-miR-409-3p & URS00002915C8_9606 & GAAUGUUGCUCGGUGAACCCCU & {$[54,65]$} \\
\hline & hsa-miR-409-5p & URS0000081E1F_9606 & AGGUUACCCGAGCAACUUUGCAU & {$[54,65]$} \\
\hline & hsa-miR-410-3p & URS000047E765_9606 & AAUAUAACACAGAUGGCCUGU & {$[54]$} \\
\hline & $\begin{array}{l}\text { hsa-miR-539-5p } \\
\text { (hsa-miR-539) }\end{array}$ & URS00003E59B7_9606 & GGAGAAAUUAUCCUUGGUGUGU & {$[65]$} \\
\hline mir-155 & $\begin{array}{l}\text { hsa-miR-155-5p } \\
\text { (hsa-miR-155) [65] }\end{array}$ & URS0000338542_9606 & UUAAUGCUAAUCGUGAUAGGGGU & {$[65,71]$} \\
\hline \multirow[t]{2}{*}{$\operatorname{mir}-17$} & $\begin{array}{l}\text { hsa-miR-106a-5p } \\
\text { (hsa-miR-106a) }\end{array}$ & URS00003FE4D4_9606 & AAAAGUGCUUACAGUGCAGGUAG & {$[65]$} \\
\hline & $\begin{array}{l}\text { hsa-miR-106b-3p } \\
\text { (hsa-miR-106b*) }\end{array}$ & URS0000384021_9606 & CCGCACUGUGGGUACUUGCUGC & {$[65]$} \\
\hline
\end{tabular}


Table 2 (continued)

\begin{tabular}{|c|c|c|c|c|}
\hline Family & Name & RNAcental & Sequence & Ref. \\
\hline & $\begin{array}{l}\text { hsa-miR-106b-5p } \\
\text { (hsa-miR-106b) }\end{array}$ & URS00004449AE_9606 & UAAAGUGCUGACAGUGCAGAU & {$[65]$} \\
\hline & $\begin{array}{l}\text { hsa-miR-17-3p } \\
\text { (hsa-miR-17*) }\end{array}$ & URS00004636A3_9606 & ACUGCAGUGAAGGCACUUGUAG & {$[65]$} \\
\hline & $\begin{array}{l}\text { hsa-miR-17-5p } \\
\text { (hsa-miR-17) }\end{array}$ & URS00002075FA_9606 & CAAAGUGCUUACAGUGCAGGUAG & {$[65]$} \\
\hline & $\begin{array}{l}\text { hsa-miR-18a-3p } \\
\text { (hsa-miR-18a*) }\end{array}$ & URS00004131FE_9606 & ACUGCCCUAAGUGCUCCUUCUGG & {$[65]$} \\
\hline & $\begin{array}{l}\text { hsa-miR-18a-5p } \\
\text { (hsa-miR-18a) }\end{array}$ & URS000035CC3E_9606 & UAAGGUGCAUCUAGUGCAGAUAG & {$[65]$} \\
\hline & $\begin{array}{l}\text { hsa-miR-18b-5p } \\
\text { (hsa-miR-18b) }\end{array}$ & URS00004565E5_9606 & UAAGGUGCAUCUAGUGCAGUUAG & {$[65]$} \\
\hline & $\begin{array}{l}\text { hsa-miR-20a-3p } \\
\text { (hsa-miR-20a*) }\end{array}$ & URS0000042E1F_9606 & ACUGCAUUAUGAGCACUUAAAG & {$[65]$} \\
\hline & $\begin{array}{l}\text { hsa-miR-20a-5p } \\
\text { (hsa-miR-20a) [65] }\end{array}$ & URS0000574A2C_9606 & UAAAGUGCUUAUAGUGCAGGUAG & {$[65,72]$} \\
\hline \multirow[t]{3}{*}{ mir-17 } & $\begin{array}{l}\text { hsa-miR-20b-5p } \\
\text { (hsa-miR-20b) }\end{array}$ & URS00002B3783_9606 & CAAAGUGCUCAUAGUGCAGGUAG & {$[65]$} \\
\hline & $\begin{array}{l}\text { hsa-miR-93-3p } \\
\text { (hsa-miR-93*) }\end{array}$ & URS00000FB1B1_9606 & ACUGCUGAGCUAGCACUUCCCG & {$[65]$} \\
\hline & hsa-miR-93-5p & URS0000149452_9606 & CAAAGUGCUGUUCGUGCAGGUAG & {$[54,59]$} \\
\hline \multirow[t]{6}{*}{$\operatorname{mir}-181$} & $\begin{array}{l}\text { hsa-miR-181a-2-3p } \\
\text { (hsa-miR-181a-2*) }\end{array}$ & URS0000241987_9606 & ACCACUGACCGUUGACUGUACC & {$[65]$} \\
\hline & $\begin{array}{l}\text { hsa-miR-181a-3p } \\
\text { (hsa-miR-213) }\end{array}$ & URS000003F252_9606 & ACCAUCGACCGUUGAUUGUACC & {$[65]$} \\
\hline & $\begin{array}{l}\text { hsa-miR-181a-5p } \\
\text { (hsa-miR-181a) [65] }\end{array}$ & URS00003DA300_9606 & AACAUUCAACGCUGUCGGUGAGU & {$[54,65]$} \\
\hline & hsa-miR-181b-5p & URS0000605E00_9606 & AACAUUCAUUGCUGUCGGUGGGU & {$[54]$} \\
\hline & $\begin{array}{l}\text { hsa-miR-181c-3p } \\
\text { (hsa-miR-181c*) }\end{array}$ & URS0000244A71_9606 & AACCAUCGACCGUUGAGUGGAC & {$[65]$} \\
\hline & $\begin{array}{l}\text { hsa-miR-181c-5p } \\
\text { (hsa-miR-181c) [65] }\end{array}$ & URS000018C928_9606 & AACAUUCAACCUGUCGGUGAGU & {$[54,65]$} \\
\hline $\operatorname{mir}-182$ & $\begin{array}{l}\text { hsa-miR-182-5p } \\
\text { (hsa-miR-182) }\end{array}$ & URS00001CC379_9606 & UUUGGCAAUGGUAGAACUCACACU & {$[65]$} \\
\hline mir-1825 & hsa-miR-1825 & URS000075AF4A_9606 & UCCAGUGCCCUCCUCUCC & {$[65]$} \\
\hline \multirow[t]{2}{*}{$\operatorname{mir}-183$} & hsa-miR-183-3p & URS0000345DEB_9606 & GUGAAUUACCGAAGGGCCAUAA & {$[65]$} \\
\hline & $\begin{array}{l}\text { hsa-miR-183-5p } \\
\text { (hsa-miR-183) }\end{array}$ & URS0000528CBC_9606 & UAUGGCACUGGUAGAAUUCACU & {$[65]$} \\
\hline $\operatorname{mir}-184$ & hsa-miR-184 & URS0000543D82_9606 & UGGACGGAGAACUGAUAAGGGU & {$[65]$} \\
\hline \multirow[t]{2}{*}{ mir-185 } & hsa-miR-185-3p & URS00002367FA_9606 & AGGGGCUGGCUUUCCUCUGGUC & {$[67]$} \\
\hline & $\begin{array}{l}\text { hsa-miR-185-5p } \\
\text { (hsa-miR-185) }\end{array}$ & URS00004176D4_9606 & UGGAGAGAAAGGCAGUUCCUGA & {$[65,70]$} \\
\hline mir-186 & $\begin{array}{l}\text { hsa-miR-186-5p } \\
\text { (hsa-miR-186) } \\
{[65,70]}\end{array}$ & URS000040DCFF_9606 & CAAAGAAUUCUCCUUUUGGGCU & {$[54,65,70]$} \\
\hline \multirow[t]{3}{*}{$\operatorname{mir}-188$} & hsa-miR-532-3p & URS00004B4B85_9606 & CCUCCCACACCCAAGGCUUGCA & {$[65,67]$} \\
\hline & $\begin{array}{l}\text { hsa-miR-532-5p } \\
\text { (hsa-miR-532) }\end{array}$ & URS00004E8341_9606 & CAUGCCUUGAGUGUAGGACCGU & {$[65,70]$} \\
\hline & $\begin{array}{l}\text { hsa-miR-660-5p } \\
\text { (hsa-miR-660) }\end{array}$ & URS0000116A70_9606 & UACCCAUUGCAUAUCGGAGUUG & {$[65,70]$} \\
\hline \multirow[t]{3}{*}{ mir-19 } & $\begin{array}{l}\text { hsa-miR-19a-3p } \\
\text { (hsa-miR-19a) }\end{array}$ & URS000006FDD4_9606 & UGUGCAAAUCUAUGCAAAACUGA & {$[65,70]$} \\
\hline & $\begin{array}{l}\text { hsa-miR-19b-1-5p } \\
\text { (hsa-miR-19b-1*) }\end{array}$ & URS00001B9622_9606 & AGUUUUGCAGGUUUGCAUCCAGC & {$[65]$} \\
\hline & $\begin{array}{l}\text { hsa-miR-19b-3p } \\
\text { (hsa-miR-19b) }[65,70]\end{array}$ & URS000013D17D_9606 & UGUGCAAAUCCAUGCAAAACUGA & {$[65,66,70,72]$} \\
\hline $\operatorname{mir}-190$ & $\begin{array}{l}\text { hsa-miR-190a-5p } \\
\text { (hsa-miR-190) }\end{array}$ & URS0000520927_9606 & UGAUAUGUUUGAUAUAUUAGGU & {$[65]$} \\
\hline \multirow[t]{2}{*}{ mir-1908 } & hsa-miR-1908-3p & URS000075E4A7_9606 & CCGGCCGCCGGCUCCGCCCCG & {$[54]$} \\
\hline & hsa-miR-1908-5p & URS00002373FD_9606 & CGGCGGGGACGGCGAUUGGUC & [67] \\
\hline
\end{tabular}


Table 2 (continued)

\begin{tabular}{|c|c|c|c|c|}
\hline Family & Name & RNAcental & Sequence & Ref. \\
\hline \multirow[t]{2}{*}{ mir-191 } & $\begin{array}{l}\text { hsa-miR-191-3p } \\
\text { (hsa-miR-191*) }\end{array}$ & URS00002B2B5C_9606 & GCUGCGCUUGGAUUUCGUCCCC & {$[65]$} \\
\hline & $\begin{array}{l}\text { hsa-miR-191-5p } \\
\text { (hsa-miR-191) }[65,70]\end{array}$ & URS00005C2E31_9606 & CAACGGAAUCCCAAAAGCAGCUG & {$[11,54,65,66,70]$} \\
\hline mir-1914 & hsa-miR-1914-3p & URS000075E34C_9606 & GGAGGGGUCCCGCACUGGGAGG & {$[67]$} \\
\hline mir-1915 & hsa-miR-1915-3p & URS000039BFD2_9606 & CCCCAGGGCGACGCGGCGGG & {$[12,72]$} \\
\hline \multirow[t]{2}{*}{ mir-192 } & $\begin{array}{l}\text { hsa-miR-192-3p } \\
\text { (hsa-miR-192*) }\end{array}$ & URS00000B59A2_9606 & CUGCCAAUUCCAUAGGUCACAG & {$[65]$} \\
\hline & $\begin{array}{l}\text { hsa-miR-192-5p } \\
\text { (hsa-miR-192) [65] }\end{array}$ & URS0000155642_9606 & CUGACCUAUGAAUUGACAGCC & {$[54,65]$} \\
\hline \multirow[t]{4}{*}{ mir-193 } & hsa-miR-193a-3p & URS00005DBAF3_9606 & AACUGGCCUACAAAGUCCCAGU & {$[65]$} \\
\hline & hsa-miR-193a-5p & URS0000367985_9606 & UGGGUCUUUGCGGGCGAGAUGA & {$[54,65,66]$} \\
\hline & $\begin{array}{l}\text { hsa-miR-193b-3p } \\
\text { (hsa-miR-193b) }\end{array}$ & URS00000AA464_9606 & AACUGGCCCUCAAAGUCCCGCU & {$[65]$} \\
\hline & $\begin{array}{l}\text { hsa-miR-193b-5p } \\
\text { (hsa-miR-193b*) [65] }\end{array}$ & URS00000E1DC5_9606 & CGGGGUUUUGAGGGCGAGAUGA & {$[53,65]$} \\
\hline mir-194 & $\begin{array}{l}\text { hsa-miR-194-5p } \\
\text { (hsa-miR-194) }\end{array}$ & URS000029C2DC_9606 & UGUAACAGCAACUCCAUGUGGA & {$[65]$} \\
\hline \multirow[t]{2}{*}{ mir-196 } & hsa-miR-196a-5p & URS00000DA6A7_9606 & UAGGUAGUUUCAUGUUGUUGGG & {$[53,59]$} \\
\hline & $\begin{array}{l}\text { hsa-miR-196b-5p } \\
\text { (hsa-miR-196b) [65] }\end{array}$ & URS0000611746_9606 & UAGGUAGUUUCCUGUUGUUGGG & {$[53,65]$} \\
\hline \multirow[t]{2}{*}{ mir-197 } & $\begin{array}{l}\text { hsa-miR-197-3p } \\
\text { (hsa-miR-197) }\end{array}$ & URS000061E740_9606 & UUCACCACCUUCUCCACCCAGC & {$[65]$} \\
\hline & hsa-miR-197-5p & URS000020E2DD_9606 & CGGGUAGAGAGGGCAGUGGGAGG & [67] \\
\hline mir-1972 & hsa-miR-1972 & URS000042A1A2_9606 & UCAGGCCAGGCACAGUGGCUCA & {$[54,66]$} \\
\hline mir-198 & hsa-miR-198 & URS000075CAC3_9606 & GGUCCAGAGGGGAGAUAGGUUC & {$[65]$} \\
\hline \multirow[t]{3}{*}{ mir-199 } & $\begin{array}{l}\text { hsa-miR-199a-5p } \\
\text { (hsa-miR-199a) [65] }\end{array}$ & URS0000554A4F_9606 & CCCAGUGUUCAGACUACCUGUUC & {$[53,54,65]$} \\
\hline & $\begin{array}{l}\text { hsa-miR-199b-3p } \\
\text { hsa-miR-199a-3p }\end{array}$ & URS00003F2D94_9606 & ACAGUAGUCUGCACAUUGGUUA & {$[53,65,66,72]$} \\
\hline & $\begin{array}{l}\text { hsa-miR-199b-5p } \\
\text { (hsa-miR-199b) } \\
{[65,67]}\end{array}$ & URS0000029EBD_9606 & CCCAGUGUUUAGACUAUCUGUUC & {$[53,65,67]$} \\
\hline mir-203 & hsa-miR-203a-3p & $\begin{array}{l}\text { URS00004DA9DB_ } \\
9606\end{array}$ & GUGAAAUGUUUAGGACCACUAG & {$[65]$} \\
\hline \multirow[t]{2}{*}{ mir-204 } & hsa-miR-204-3p & URS000059A01D_9606 & GCUGGGAAGGCAAAGGGACGU & {$[54]$} \\
\hline & $\begin{array}{l}\text { hsa-miR-204-5p } \\
\text { (hsa-miR-204) [65] }\end{array}$ & URS000029D9F1_9606 & UUCCCUUUGUCAUCCUAUGCCU & {$[54,65]$} \\
\hline mir-205 & $\begin{array}{l}\text { hsa-miR-205-5p } \\
\quad \text { (hsa-miR-205) [65, 68] }\end{array}$ & URS0000446722_9606 & UCCUUCAUUCCACCGGAGUCUG & {$[54,65,68]$} \\
\hline \multirow[t]{2}{*}{$\operatorname{mir}-21$} & $\begin{array}{l}\text { hsa-miR-21-3p } \\
\text { (hsa-miR-21*) [65] }\end{array}$ & URS000009262D_9606 & CAACACCAGUCGAUGGGCUGU & {$[54,65]$} \\
\hline & $\begin{array}{l}\text { hsa-miR-21-5p } \\
\text { (hsa-miR-21) } \\
\quad[59,65,67,74]\end{array}$ & URS000039ED8D_9606 & UAGCUUAUCAGACUGAUGUUGA & $\begin{array}{l}{[11,54,59,65-67,} \\
\quad 71,73,74]\end{array}$ \\
\hline $\operatorname{mir}-210$ & hsa-miR-210-5p & URS000075D16F_9606 & AGCCCCUGCCCACCGCACACUG & {$[67]$} \\
\hline \multirow[t]{2}{*}{ mir-214 } & $\begin{array}{l}\text { hsa-miR-214-3p } \\
\text { (hsa-miR-214) [65] }\end{array}$ & URS00002C11C3_9606 & ACAGCAGGCACAGACAGGCAGU & {$[12,65,66]$} \\
\hline & $\begin{array}{l}\text { hsa-miR-214-5p } \\
\text { (hsa-miR-214*) }\end{array}$ & URS00004DAA89_9606 & UGCCUGUCUACACUUGCUGUGC & {$[65]$} \\
\hline $\operatorname{mir}-216$ & $\begin{array}{l}\text { hsa-miR-216a-5p } \\
\text { (hsa-miR-216a) }\end{array}$ & URS0000318E24_9606 & UAAUCUCAGCUGGCAACUGUGA & {$[65]$} \\
\hline \multirow[t]{2}{*}{$\operatorname{mir}-218$} & $\begin{array}{l}\text { hsa-miR-218-2-3p } \\
\text { (hsa-miR-218-2*) }\end{array}$ & URS00001F9A0F_9606 & CAUGGUUCUGUCAAGCACCGCG & {$[65]$} \\
\hline & hsa-miR-218-5p & URS000020D84A_9606 & UUGUGCUUGAUCUAACCAUGU & {$[54]$} \\
\hline $\operatorname{mir}-219$ & $\begin{array}{l}\text { hsa-miR-219a-5p } \\
\text { (hsa-miR-219) }\end{array}$ & URS0000565C8D_9606 & UGAUUGUCCAAACGCAAUUCU & {$[65]$} \\
\hline mir-22 & hsa-miR-22-3p & URS0000096022_9606 & AAGCUGCCAGUUGAAGAACUGU & {$[11,12,53,54,65]$} \\
\hline
\end{tabular}


Table 2 (continued)

\begin{tabular}{|c|c|c|c|c|}
\hline Family & Name & RNAcental & Sequence & Ref. \\
\hline & $\begin{array}{l}\text { hsa-miR-22-5p } \\
\text { (hsa-miR-22*) [65] }\end{array}$ & URS0000142DC3_9606 & AGUUCUUCAGUGGCAAGCUUUA & {$[65,70]$} \\
\hline \multirow[t]{3}{*}{ mir-221 } & $\begin{array}{l}\text { hsa-miR-221-3p } \\
\text { (hsa-miR-221) [65] }\end{array}$ & URS0000170CF4_9606 & AGCUACAUUGUCUGCUGGGUUUC & {$[12,54,59,65,66,69]$} \\
\hline & $\begin{array}{l}\text { hsa-miR-222-3p } \\
\quad \text { (hsa-miR-222) } \\
{[63,64,66]}\end{array}$ & URS00002C6949_9606 & AGCUACAUCUGGCUACUGGGU & $\begin{array}{l}{[11,12,54,59,64,} \\
\quad 65,70]\end{array}$ \\
\hline & $\begin{array}{l}\text { hsa-miR-222-5p } \\
\text { (hsa-miR-222*) }\end{array}$ & URS0000153377_9606 & CUCAGUAGCCAGUGUAGAUCCU & {$[65]$} \\
\hline \multirow[t]{2}{*}{ mir-223 } & $\begin{array}{l}\text { hsa-miR-223-3p } \\
\text { (hsa-miR-223) }\end{array}$ & URS00000B7E30_9606 & UGUCAGUUUGUCAAAUACCCCA & {$[65]$} \\
\hline & $\begin{array}{l}\text { hsa-miR-223-5p } \\
\text { (hsa-miR-223*) }\end{array}$ & URS0000485CBB_9606 & CGUGUAUUUGACAAGCUGAGUU & {$[65]$} \\
\hline $\operatorname{mir}-224$ & $\begin{array}{l}\text { hsa-miR-224-5p } \\
\text { (hsa-miR-224) }\end{array}$ & URS00002BBD4E_9606 & CAAGUCACUAGUGGUUCCGUU & {$[65]$} \\
\hline \multirow[t]{2}{*}{$\operatorname{mir}-23$} & $\begin{array}{l}\text { hsa-miR-23a-3p } \\
\text { (hsa-miR-23a) [64, 65] }\end{array}$ & URS00005540D2_9606 & AUCACAUUGCCAGGGAUUUCC & {$[12,59,64-66,69,72]$} \\
\hline & hsa-miR-23b-3p & URS0000183BED_9606 & AUCACAUUGCCAGGGAUUACC & {$[12,54,59,66]$} \\
\hline \multirow[t]{3}{*}{$\operatorname{mir}-24$} & $\begin{array}{l}\text { hsa-miR-24-1-5p } \\
\text { (hsa-miR-24-1*) }\end{array}$ & URS00002D0FC3_9606 & UGCCUACUGAGCUGAUAUCAGU & {$[65]$} \\
\hline & $\begin{array}{l}\text { hsa-miR-24-2-5p } \\
\text { (hsa-miR-24-2*) }\end{array}$ & URS00001DEE11_9606 & UGCCUACUGAGCUGAAACACAG & {$[65]$} \\
\hline & $\begin{array}{l}\text { hsa-miR-24-3p } \\
\text { (hsa-miR-24) [65] }\end{array}$ & URS000059273E_9606 & UGGCUCAGUUCAGCAGGAACAG & {$[12,53,59,65,66]$} \\
\hline \multirow[t]{5}{*}{$\operatorname{mir}-25$} & $\begin{array}{l}\text { hsa-miR-25-3p } \\
\text { (hsa-miR-25) [65] }\end{array}$ & URS00004F9744_9606 & CAUUGCACUUGUCUCGGUCUGA & {$[54,65,66]$} \\
\hline & $\begin{array}{l}\text { hsa-miR-25-5p } \\
\text { (hsa-miR-25*) }\end{array}$ & URS00001A9746_9606 & AGGCGGAGACUUGGGCAAUUG & {$[65]$} \\
\hline & $\begin{array}{l}\text { hsa-miR-92a-3p } \\
\text { (hsa-miR-92a) [65] }\end{array}$ & URS00003768C5_9606 & UAUUGCACUUGUCCCGGCCUGU & {$[11,54,65,66]$} \\
\hline & hsa-miR-92b-3p & URS000025576D_9606 & UAUUGCACUCGUCCCGGCCUCC & {$[11,54]$} \\
\hline & $\begin{array}{l}\text { hsa-miR-92b-5p } \\
\text { (hsa-miR-92b*) }\end{array}$ & URS00001A7F58_9606 & AGGGACGGGACGCGGUGCAGUG & {$[65]$} \\
\hline \multirow[t]{4}{*}{$\operatorname{mir}-26$} & $\begin{array}{l}\text { hsa-miR-26b-3p } \\
\text { (hsa-miR-26b*) }\end{array}$ & URS000021C6A8_9606 & CCUGUUCUCCAUUACUUGGCU & {$[65]$} \\
\hline & $\begin{array}{l}\text { hsa-miR-26b-5p } \\
\text { (hsa-miR-26b) }\end{array}$ & URS0000316FA5_9606 & UUCAAGUAAUUCAGGAUAGGU & {$[65]$} \\
\hline & $\begin{array}{l}\text { hsa-miR-26a-1-3p } \\
\text { (hsa-miR-26a-1*) }\end{array}$ & URS00000C0D3F_9606 & CCUAUUCUUGGUUACUUGCACG & {$[65]$} \\
\hline & $\begin{array}{l}\text { hsa-miR-26a-5p } \\
\text { (hsa-miR-26a) [65] }\end{array}$ & URS000019B0F7_9606 & UUCAAGUAAUCCAGGAUAGGCU & {$[11,54,65,69]$} \\
\hline \multirow[t]{4}{*}{$\operatorname{mir}-27$} & $\begin{array}{l}\text { hsa-miR-27a-3p } \\
\text { (hsa-miR-27a) [65] }\end{array}$ & URS00003B95DA_9606 & UUCACAGUGGCUAAGUUCCGC & {$[12,53,65]$} \\
\hline & $\begin{array}{l}\text { hsa-miR-27a-5p } \\
\text { (hsa-miR-27a*) [65] }\end{array}$ & URS00001B341F_9606 & AGGGCUUAGCUGCUUGUGAGCA & {$[65,70]$} \\
\hline & $\begin{array}{l}\text { hsa-miR-27b-3p } \\
\text { (hsa-miR-27b) [65] }\end{array}$ & URS000059311D_9606 & UUCACAGUGGCUAAGUUCUGC & {$[54,65]$} \\
\hline & $\begin{array}{l}\text { hsa-miR-27b-5p } \\
\text { (hsa-miR-27b*) }\end{array}$ & URS0000330617_9606 & AGAGCUUAGCUGAUUGGUGAAC & {$[65]$} \\
\hline \multirow[t]{5}{*}{$\operatorname{mir}-28$} & $\begin{array}{l}\text { hsa-miR-151a-3p } \\
\text { (hsa-miR-151-3p) [65] }\end{array}$ & URS000016C318_9606 & CUAGACUGAAGCUCCUUGAGG & {$[11,54,65]$} \\
\hline & $\begin{array}{l}\text { hsa-miR-151a-5p } \\
\text { (hsa-miR-151-5p) [65] }\end{array}$ & URS00005F8E5B_9606 & UCGAGGAGCUCACAGUCUAGU & {$[54,65]$} \\
\hline & hsa-miR-151b & URS00003E6479_9606 & UCGAGGAGCUCACAGUCU & {$[54]$} \\
\hline & hsa-miR-28-3p & URS00001799A3_9606 & CACUAGAUUGUGAGCUCCUGGA & {$[54,65]$} \\
\hline & $\begin{array}{l}\text { hsa-miR-28-5p } \\
\text { (hsa-miR-28) }\end{array}$ & URS00003E47B1_9606 & AAGGAGCUCACAGUCUAUUGAG & {$[65]$} \\
\hline $\operatorname{mir}-2861$ & hsa-miR-2861 & URS00003B13B8_9606 & GGGGCCUGGCGGUGGGCGG & {$[72]$} \\
\hline $\operatorname{mir}-29$ & hsa-miR-29a-3p & URS00002F4D78_9606 & UAGCACCAUCUGAAAUCGGUUA & {$[54,65]$} \\
\hline
\end{tabular}


Table 2 (continued)

\begin{tabular}{|c|c|c|c|c|}
\hline Family & Name & RNAcental & Sequence & Ref. \\
\hline & $\begin{array}{l}\text { hsa-miR-29a-5p } \\
\text { (hsa-miR-29a*) [65] }\end{array}$ & URS0000076995_9606 & ACUGAUUUCUUUUGGUGUUCAG & {$[65,70]$} \\
\hline & $\begin{array}{l}\text { hsa-miR-29b-1-5p } \\
\text { (hsa-miR-29b-1*) }\end{array}$ & URS00001123BD_9606 & GCUGGUUUCAUAUGGUGGUUUAGA & {$[65]$} \\
\hline & $\begin{array}{l}\text { hsa-miR-29b-2-5p } \\
\text { (hsa-miR-29b-2*) }\end{array}$ & URS0000403C02_9606 & CUGGUUUCACAUGGUGGCUUAG & {$[65]$} \\
\hline & $\begin{array}{l}\text { hsa-miR-29b-3p } \\
\text { (hsa-miR-29b) [65] }\end{array}$ & URS000024463E_9606 & UAGCACCAUUUGAAAUCAGUGUU & {$[54,65]$} \\
\hline & $\begin{array}{l}\text { hsa-miR-29c-3p } \\
\text { (hsa-miR-29c) [65] }\end{array}$ & URS0000272A3D_9606 & UAGCACCAUUUGAAAUCGGUUA & {$[54,65]$} \\
\hline $\operatorname{mir}-296$ & $\begin{array}{l}\text { hsa-miR-296-5p } \\
\text { (hsa-miR-296) [65] }\end{array}$ & URS00001C3AC1_9606 & AGGGCCCCCCCUCAAUCCUGU & {$[65,67]$} \\
\hline \multirow[t]{2}{*}{$\operatorname{mir}-299$} & hsa-miR-299-3p & URS00003B1F5C_9606 & UAUGUGGGAUGGUAAACCGCUU & {$[54,65]$} \\
\hline & hsa-miR-299-5p & URS000017DBB8_9606 & UGGUUUACCGUCCCACAUACAU & {$[65]$} \\
\hline \multirow[t]{8}{*}{$\operatorname{mir}-30$} & hsa-miR-30a-3p & URS0000065D58_9606 & CUUUCAGUCGGAUGUUUGCAGC & {$[65]$} \\
\hline & hsa-miR-30a-5p & URS000043D1A9_9606 & UGUAAACAUCCUCGACUGGAAG & {$[54,65]$} \\
\hline & $\begin{array}{l}\text { hsa-miR-30b-5p } \\
\text { (hsa-miR-30b) }\end{array}$ & URS00005165DA_9606 & UGUAAACAUCCUACACUCAGCU & {$[65,70]$} \\
\hline & $\begin{array}{l}\text { hsa-miR-30c-5p } \\
\text { (hsa-miR-30c) [65] }\end{array}$ & URS000019907A_9606 & UGUAAACAUCCUACACUCUCAGC & {$[54,65]$} \\
\hline & $\begin{array}{l}\text { hsa-miR-30d-3p } \\
\text { (hsa-miR-30d*) }\end{array}$ & URS00004B2A47_9606 & CUUUCAGUCAGAUGUUUGCUGC & {$[65]$} \\
\hline & $\begin{array}{l}\text { hsa-miR-30d-5p } \\
\text { (hsa-miR-30d) [65] }\end{array}$ & URS000005CF5F_9606 & UGUAAACAUCCCCGACUGGAAG & {$[54,65]$} \\
\hline & hsa-miR-30e-3p & URS00004DC6A5_9606 & CUUUCAGUCGGAUGUUUACAGC & {$[65,70]$} \\
\hline & hsa-miR-30e-5p & URS00001DE669_9606 & UGUAAACAUCCUUGACUGGAAG & [54] \\
\hline \multirow[t]{3}{*}{ mir-302 } & $\begin{array}{l}\text { hsa-miR-302a-3p } \\
\text { (hsa-miR-302a) }\end{array}$ & URS0000070CD2_9606 & UAAGUGCUUCCAUGUUUUGGUGA & {$[65]$} \\
\hline & $\begin{array}{l}\text { hsa-miR-302c-3p } \\
\text { (hsa-miR-302c) }\end{array}$ & URS000027080C_9606 & UAAGUGCUUCCAUGUUUCAGUGG & {$[65]$} \\
\hline & $\begin{array}{l}\text { hsa-miR-302d-3p } \\
\text { (hsa-miR-302d) }\end{array}$ & URS000041E949_9606 & UAAGUGCUUCCAUGUUUGAGUGU & {$[65]$} \\
\hline \multirow[t]{2}{*}{$\operatorname{mir}-31$} & $\begin{array}{l}\text { hsa-miR-31-3p } \\
\text { (hsa-miR-31*) }\end{array}$ & URS00002A291B_9606 & UGCUAUGCCAACAUAUUGCCAU & {$[65]$} \\
\hline & $\begin{array}{l}\text { hsa-miR-31-5p } \\
\text { (hsa-miR-31) [65] }\end{array}$ & URS00005416E3_9606 & AGGCAAGAUGCUGGCAUAGCU & {$[12,59,65]$} \\
\hline $\operatorname{mir}-3180$ & hsa-miR-3180-3p & URS00002C4233_9606 & UGGGGCGGAGCUUCCGGAGGCC & {$[67]$} \\
\hline $\operatorname{mir}-32$ & $\begin{array}{l}\text { hsa-miR-32-5p } \\
\text { (hsa-miR-32) }\end{array}$ & URS00004C47FB_9606 & UAUUGCACAUUACUAAGUUGCA & {$[65]$} \\
\hline \multirow[t]{3}{*}{ mir-320 } & $\begin{array}{l}\text { hsa-miR-320a-3p" } \\
\text { (hsa-miR-320) [65] }\end{array}$ & URS00003CF1AD_9606 & AAAAGCUGGGUUGAGAGGGCGA & {$[54,65]$} \\
\hline & hsa-miR-320b & URS000058BF17_9606 & AAAAGCUGGGUUGAGAGGGCAA & {$[54,65]$} \\
\hline & hsa-miR-320c & URS0000010D30_9606 & AAAAGCUGGGUUGAGAGGGU & {$[54]$} \\
\hline \multirow[t]{2}{*}{ mir-322 } & $\begin{array}{l}\text { hsa-miR-424-3p } \\
\text { (hsa-miR-424*) [65] }\end{array}$ & URS00002BCF86_9606 & CAAAACGUGAGGCGCUGCUAU & {$[54,65]$} \\
\hline & $\begin{array}{l}\text { hsa-miR-424-5p } \\
\text { (hsa-miR-424) }\end{array}$ & URS00000F0F49_9606 & CAGCAGCAAUUCAUGUUUUGAA & {$[65]$} \\
\hline \multirow[t]{2}{*}{$\operatorname{mir}-324$} & hsa-miR-324-3p & URS00004390F6_9606 & ACUGCCCCAGGUGCUGCUGG & {$[65,70]$} \\
\hline & hsa-miR-324-5p & URS000075BEBE_9606 & CGCAUCCCCUAGGGCAUUGGUG & {$[65]$} \\
\hline $\operatorname{mir}-326$ & hsa-miR-326 & URS00000A939F_9606 & CCUCUGGGCCCUUCCUCCAG & {$[65]$} \\
\hline mir-329 & hsa-miR-543 & URS000019F055_9606 & AAACAUUCGCGGUGCACUUCUU & {$[65]$} \\
\hline \multirow[t]{3}{*}{$\operatorname{mir}-33$} & $\begin{array}{l}\text { hsa-miR-33a-3p } \\
\text { (hsa-miR-33a*) }\end{array}$ & URS00003E3B82_9606 & CAAUGUUUCCACAGUGCAUCAC & {$[65]$} \\
\hline & $\begin{array}{l}\text { hsa-miR-33a-5p } \\
\text { (hsa-miR-33a) }\end{array}$ & URS0000483184_9606 & GUGCAUUGUAGUUGCAUUGCA & {$[65]$} \\
\hline & $\begin{array}{l}\text { hsa-miR-33b-5p } \\
\text { (hsa-miR-33b) }\end{array}$ & URS00004C8DD5_9606 & GUGCAUUGCUGUUGCAUUGC & {$[65]$} \\
\hline mir-330 & hsa-miR-330-3p & URS000007A060_9606 & GCAAAGCACACGGCCUGCAGAGA & {$[65]$} \\
\hline
\end{tabular}


Table 2 (continued)

\begin{tabular}{|c|c|c|c|c|}
\hline Family & Name & RNAcental & Sequence & Ref. \\
\hline & (hsa-miR-330) & & & \\
\hline & hsa-miR-330-5p & URS00003380C1_9606 & UCUCUGGGCCUGUGUCUUAGGC & {$[65]$} \\
\hline \multirow[t]{2}{*}{ mir-331 } & $\begin{array}{l}\text { hsa-miR-331-3p } \\
\text { (hsa-miR-331) }\end{array}$ & URS00003DDE27_9606 & GCCCCUGGGCCUAUCCUAGAA & {$[65]$} \\
\hline & hsa-miR-331-5p & URS00001597DC_9606 & CUAGGUAUGGUCCCAGGGAUCC & {$[65]$} \\
\hline \multirow[t]{2}{*}{ mir-335 } & $\begin{array}{l}\text { hsa-miR-335-3p } \\
\text { (hsa-miR-335*) }\end{array}$ & URS00005092C2_9606 & UUUUUCAUUAUUGCUCCUGACC & {$[65]$} \\
\hline & $\begin{array}{l}\text { hsa-miR-335-5p } \\
\text { (hsa-miR-335) }\end{array}$ & URS0000237AF9_9606 & UCAAGAGCAAUAACGAAAAAUGU & {$[65]$} \\
\hline \multirow[t]{2}{*}{ mir-337 } & hsa-miR-337-3p & URS0000564D66_9606 & CUCCUAUAUGAUGCCUUUCUUC & {$[65]$} \\
\hline & hsa-miR-337-5p & URS0000306C70_9606 & GAACGGCUUCAUACAGGAGUU & {$[65]$} \\
\hline $\operatorname{mir}-338$ & hsa-miR-338-3p & URS00000254A6_9606 & UCCAGCAUCAGUGAUUUUGUUG & {$[54,65]$} \\
\hline \multirow[t]{2}{*}{ mir-339 } & hsa-miR-339-3p & URS000055B190_9606 & UGAGCGCCUCGACGACAGAGCCG & {$[65]$} \\
\hline & hsa-miR-339-5p & URS000003FD55_9606 & UCCCUGUCCUCCAGGAGCUCACG & {$[54]$} \\
\hline \multirow[t]{4}{*}{$\operatorname{mir}-34$} & $\begin{array}{l}\text { hsa-miR-34a-3p } \\
\text { (hsa-miR-34a*) }\end{array}$ & URS00000EED18_9606 & CAAUCAGCAAGUAUACUGCCCU & {$[65]$} \\
\hline & $\begin{array}{l}\text { hsa-miR-34a-5p } \\
\text { (hsa-miR-34a) [65] }\end{array}$ & URS000030BD69_9606 & UGGCAGUGUCUUAGCUGGUUGU & {$[65,71]$} \\
\hline & $\begin{array}{l}\text { hsa-miR-34b-3p } \\
\text { (hsa-miR-34b) }\end{array}$ & URS000027352D_9606 & CAAUCACUAACUCCACUGCCAU & {$[65]$} \\
\hline & $\begin{array}{l}\text { hsa-miR-34c-5p } \\
\text { (hsa-miR-34c) }\end{array}$ & URS00002C7B2B_9606 & AGGCAGUGUAGUUAGCUGAUUGC & {$[64,65]$} \\
\hline \multirow[t]{2}{*}{ mir-340 } & $\begin{array}{l}\text { hsa-miR-340-3p } \\
\text { (hsa-miR-340*) }\end{array}$ & URS000048521E_9606 & UCCGUCUCAGUUACUUUAUAGC & {$[65]$} \\
\hline & $\begin{array}{l}\text { hsa-miR-340-5p } \\
\text { (hsa-miR-340) }\end{array}$ & URS0000007FBA_9606 & UUAUAAAGCAAUGAGACUGAUU & {$[65]$} \\
\hline \multirow[t]{2}{*}{ mir-342 } & hsa-miR-342-3p & URS0000148B91_9606 & UCUCACACAGAAAUCGCACCCGU & {$[65]$} \\
\hline & hsa-miR-342-5p & URS00005A8080_9606 & AGGGGUGCUAUCUGUGAUUGA & {$[65]$} \\
\hline mir-345 & $\begin{array}{l}\text { hsa-miR-345-5p } \\
\text { (hsa-miR-345) }\end{array}$ & URS000005D4F5_9606 & GCUGACUCCUAGUCCAGGGCUC & {$[65]$} \\
\hline \multirow[t]{2}{*}{ mir-361 } & hsa-miR-361-3p & URS000031E6A1_9606 & UCCCCCAGGUGUGAUUCUGAUUU & {$[65]$} \\
\hline & $\begin{array}{l}\text { hsa-miR-361-5p } \\
\text { (hsa-miR-361) [65] }\end{array}$ & URS00000CF1D2_9606 & UUAUCAGAAUCUCCAGGGGUAC & {$[65,70]$} \\
\hline mir-3613 & hsa-miR-3613-3p & URS00004EAE33_9606 & ACAAAAAAAAAAGCCCAACCCUUC & {$[12]$} \\
\hline $\operatorname{mir}-3615$ & hsa-miR-3615 & URS000011166D_9606 & UCUCUCGGCUCCUCGCGGCUC & {$[54]$} \\
\hline \multirow[t]{2}{*}{ miR-362 } & hsa-miR-362-3p & URS00003A19A3_9606 & AACACACCUAUUCAAGGAUUCA & {$[64,65]$} \\
\hline & $\begin{array}{l}\text { hsa-miR-362-5p } \\
\text { (hsa-miR-362) }\end{array}$ & URS0000085F64_9606 & AAUCCUUGGAACCUAGGUGUGAGU & {$[65]$} \\
\hline $\operatorname{mir}-3648$ & hsa-miR-3648 & URS0000454FAB_9606 & AGCCGCGGGGAUCGCCGAGGG & {$[54]$} \\
\hline $\operatorname{mir}-365$ & $\begin{array}{l}\text { hsa-miR-365a-3p } \\
\text { (hsa-miR-365) }\end{array}$ & URS00003E7283_9606 & UAAUGCCCCUAAAAAUCCUUAU & {$[65]$} \\
\hline $\operatorname{mir}-3661$ & hsa-miR-3661 & URS00002CCA6E_9606 & UGACCUGGGACUCGGACAGCUG & {$[67]$} \\
\hline \multirow[t]{4}{*}{$\operatorname{mir}-368$} & $\begin{array}{l}\text { hsa-miR-376a-3p } \\
\text { (hsa-miR-376a) }\end{array}$ & URS000041E11D_9606 & AUCAUAGAGGAAAAUCCACGU & {$[65]$} \\
\hline & $\begin{array}{l}\text { hsa-miR-376a-5p } \\
\text { (hsa-miR-376a*) }\end{array}$ & URS000032A93F_9606 & GUAGAUUCUCCUUCUAUGAGUA & {$[65]$} \\
\hline & $\begin{array}{l}\text { hsa-miR-376b-3p } \\
\text { (hsa-miR-376b) }\end{array}$ & URS00003AD231_9606 & AUCAUAGAGGAAAAUCCAUGUU & {$[65]$} \\
\hline & $\begin{array}{l}\text { hsa-miR-376c-3p } \\
\text { (hsa-miR-376c) }\end{array}$ & URS00005E651E_9606 & AACAUAGAGGAAAUUCCACGU & {$[65]$} \\
\hline $\operatorname{mir}-3687$ & hsa-miR-3687 & URS0000420457_9606 & CCCGGACAGGCGUUCGUGCGACGU & {$[54,66]$} \\
\hline $\operatorname{mir}-370$ & hsa-miR-370-3p & URS00004900F1_9606 & GCCUGCUGGGGUGGAACCUGGU & {$[54]$} \\
\hline mir-374 & $\begin{array}{l}\text { hsa-miR-374a-5p } \\
\text { (hsa-miR-374) }\end{array}$ & URS000029E173_9606 & UUAUAAUACAACCUGAUAAGUG & {$[65]$} \\
\hline $\operatorname{mir}-375$ & hsa-miR-375-3p p $^{\#}$ & URS00000ED600_9606 & UUUGUUCGUUCGGCUCGCGUGA & {$[54]$} \\
\hline $\operatorname{mir}-378$ & $\begin{array}{l}\text { hsa-miR-378a-3p } \\
\text { (hsa-miR-378)[65] }\end{array}$ & URS00000451A1_9606 & ACUGGACUUGGAGUCAGAAGGC & {$[54,65]$} \\
\hline
\end{tabular}


Table 2 (continued)

\begin{tabular}{|c|c|c|c|c|}
\hline Family & Name & RNAcental & Sequence & Ref. \\
\hline \multirow[t]{4}{*}{ mir-379 } & hsa-miR-380-5p & URS000075BE5F_9606 & UGGUUGACCAUAGAACAUGCGC & {$[65]$} \\
\hline & $\begin{array}{l}\text { hsa-miR-411-3p } \\
\text { (hsa-miR-411*) }\end{array}$ & $\begin{array}{l}\text { URS000037DAEA_ } \\
9606\end{array}$ & UAUGUAACACGGUCCACUAACC & {$[65]$} \\
\hline & hsa-miR-411-5p & $\begin{array}{l}\text { URS00000C5BAA_ } \\
9606\end{array}$ & UAGUAGACCGUAUAGCGUACG & {$[54,65]$} \\
\hline & $\begin{array}{l}\text { hsa-miR-758-3p } \\
\text { (hsa-miR-758) }\end{array}$ & URS000024B619_9606 & UUUGUGACCUGGUCCACUAACC & {$[65]$} \\
\hline $\operatorname{mir}-384$ & hsa-miR-384 & URS000075DD0E_9606 & AUUCCUAGAAAUUGUUCAUA & {$[65]$} \\
\hline mir-3934 & hsa-miR-3934-5p & URS00003ACE11_9606 & UCAGGUGUGGAAACUGAGGCAG & {$[72]$} \\
\hline mir-3940 & hsa-miR-3940-5p & URS00001E8DA7_9606 & GUGGGUUGGGGCGGGCUCUG & {$[73]$} \\
\hline $\operatorname{mir}-3960$ & hsa-miR-3960 & URS00003783AB_9606 & GGCGGCGGCGGAGGCGGGGG & {$[12,54,66]$} \\
\hline $\operatorname{mir}-422$ & hsa-miR-422a & URS00003CC245_9606 & ACUGGACUUAGGGUCAGAAGGC & {$[65]$} \\
\hline \multirow[t]{2}{*}{$\operatorname{mir}-423$} & hsa-miR-423-3p & URS00000BE495_9606 & AGCUCGGUCUGAGGCCCCUCAGU & {$[54]$} \\
\hline & hsa-miR-423-5p & URS00001C8A86_9606 & UGAGGGGCAGAGAGCGAGACUUU & {$[54,65,66,69,70]$} \\
\hline \multirow[t]{2}{*}{$\operatorname{mir}-425$} & $\begin{array}{l}\text { hsa-miR-425-3p } \\
\text { (hsa-miR-425*) }\end{array}$ & URS000056B04E_9606 & AUCGGGAAUGUCGUGUCCGCCC & {$[65]$} \\
\hline & hsa-miR-425-5p & URS000048BA36_9606 & AAUGACACGAUCACUCCCGUUGA & {$[65,69]$} \\
\hline $\operatorname{mir}-431$ & $\begin{array}{l}\text { hsa-miR-431-5p } \\
\text { (hsa-miR-431) }\end{array}$ & URS000043908D_9606 & UGUCUUGCAGGCCGUCAUGCA & {$[65]$} \\
\hline $\operatorname{mir}-432$ & $\begin{array}{l}\text { hsa-miR-432-5p } \\
\text { (hsa-miR-432) }\end{array}$ & URS00001C406A_9606 & UCUUGGAGUAGGUCAUUGGGUGG & {$[65]$} \\
\hline $\operatorname{mir}-4446$ & hsa-miR-4446-3p & URS000000EF0B_9606 & CAGGGCUGGCAGUGACAUGGGU & {$[67]$} \\
\hline $\operatorname{mir}-4449$ & hsa-miR-4449 & URS00004DE2FC_9606 & CGUCCCGGGGCUGCGCGAGGCA & {$[54,67]$} \\
\hline $\operatorname{mir}-4488$ & hsa-miR-4488 & URS0000419B5A_9606 & AGGGGGCGGGCUCCGGCG & {$[12,54,66]$} \\
\hline \multirow[t]{2}{*}{ mir-449 } & $\begin{array}{l}\text { hsa-miR-449a } \\
\text { (hsa-miR-449) }\end{array}$ & URS00001F5B39_9606 & UGGCAGUGUAUUGUUAGCUGGU & {$[65]$} \\
\hline & $\begin{array}{l}\text { hsa-miR-449b-5p } \\
\text { (hsa-miR-449b) }\end{array}$ & URS00003758F0_9606 & AGGCAGUGUAUUGUUAGCUGGC & {$[65]$} \\
\hline \multirow[t]{3}{*}{$\operatorname{mir}-450$} & $\begin{array}{l}\text { hsa-miR-450a-5p } \\
\text { (hsa-miR-450a) }\end{array}$ & URS00003E5ECC_9606 & UUUUGCGAUGUGUUCCUAAUAU & {$[65]$} \\
\hline & hsa-miR-450b-3p & URS00002FF522_9606 & UUGGGAUCAUUUUGCAUCCAUA & {$[65]$} \\
\hline & hsa-miR-450b-5p & URS0000422A99_9606 & UUUUGCAAUAUGUUCCUGAAUA & {$[65]$} \\
\hline $\operatorname{mir}-452$ & $\begin{array}{l}\text { hsa-miR-452-5p } \\
\text { (hsa-miR-452) }\end{array}$ & URS0000550C66_9606 & AACUGUUUGCAGAGGAAACUGA & {$[65]$} \\
\hline \multirow[t]{2}{*}{$\operatorname{mir}-454$} & $\begin{array}{l}\text { hsa-miR-454-3p } \\
\text { (hsa-miR-454) }\end{array}$ & URS00004F77ED_9606 & UAGUGCAAUAUUGCUUAUAGGGU & {$[65]$} \\
\hline & $\begin{array}{l}\text { hsa-miR-454-5p } \\
\text { (hsa-miR-454*) }\end{array}$ & URS000031602A_9606 & ACCCUAUCAAUAUUGUCUCUGC & {$[65]$} \\
\hline \multirow[t]{2}{*}{$\operatorname{mir}-455$} & hsa-miR-455-3p & URS000022A78C_9606 & GCAGUCCAUGGGCAUAUACAC & {$[65]$} \\
\hline & $\begin{array}{l}\text { hsa-miR-455-5p } \\
\text { (hsa-miR-455) }\end{array}$ & URS00000AD002_9606 & UAUGUGCCUUUGGACUACAUCG & {$[65]$} \\
\hline \multirow[t]{2}{*}{$\operatorname{mir}-483$} & hsa-miR-483-3p & URS00000EA063_9606 & UCACUCCUCUCCUCCCGUCUU & {$[65]$} \\
\hline & hsa-miR-483-5p & URS000003575B_9606 & AAGACGGGAGGAAAGAAGGGAG & {$[65]$} \\
\hline $\operatorname{mir}-484$ & hsa-miR-484 & URS0000597BED_9606 & UCAGGCUCAGUCCCCUCCCGAU & {$[54,65,67]$} \\
\hline $\operatorname{mir}-485$ & hsa-miR-485-3p & URS000006372A_9606 & GUCAUACACGGCUCUCCUCUCU & {$[65]$} \\
\hline $\operatorname{mir}-485$ & hsa-miR-485-5p & URS00001935FA_9606 & AGAGGCUGGCCGUGAUGAAUUC & {$[65]$} \\
\hline $\operatorname{mir}-486$ & $\begin{array}{l}\text { hsa-miR-486-5p } \\
\text { (hsa-miR-486) [65] }\end{array}$ & URS00004BF1DC_9606 & UCCUGUACUGAGCUGCCCCGAG & {$[11,54,65]$} \\
\hline $\operatorname{mir}-488$ & $\begin{array}{l}\text { hsa-miR-488-3p } \\
\text { (hsa-miR-488) }\end{array}$ & $\begin{array}{l}\text { URS00001BCAC5 } \\
\quad 9606\end{array}$ & UUGAAAGGCUAUUUCUUGGUC & {$[65]$} \\
\hline $\operatorname{mir}-492$ & hsa-miR-492 & URS000032599B_9606 & AGGACCUGCGGGACAAGAUUCUU & {$[65]$} \\
\hline $\operatorname{mir}-493$ & $\begin{array}{l}\text { hsa-miR-493-3p } \\
\text { (hsa-miR-493) }\end{array}$ & URS00005E7CB2_9606 & UGAAGGUCUACUGUGUGCCAGG & {$[65]$} \\
\hline $\operatorname{mir}-497$ & $\begin{array}{l}\text { hsa-miR-497-5p } \\
\text { (hsa-miR-497) }\end{array}$ & URS00001BC212_9606 & CAGCAGCACACUGUGGUUUGU & {$[65]$} \\
\hline $\operatorname{mir}-500$ & hsa-miR-500a-5p & URS000039A052_9606 & UAAUCCUUGCUACCUGGGUGAGA & {$[65]$} \\
\hline
\end{tabular}


Table 2 (continued)

\begin{tabular}{|c|c|c|c|c|}
\hline Family & Name & RNAcental & Sequence & Ref. \\
\hline & (hsa-miR-500) & & & \\
\hline & hsa-miR-501-3p & URS00000EEE35_9606 & AAUGCACCCGGGCAAGGAUUCU & {$[65]$} \\
\hline & $\begin{array}{l}\text { hsa-miR-501-5p } \\
\text { (hsa-miR-501) }\end{array}$ & URS00001E2DBC_9606 & AAUCCUUUGUCCCUGGGUGAGA & {$[65]$} \\
\hline & hsa-miR-502-3p & URS0000601CC4_9606 & AAUGCACCUGGGCAAGGAUUCA & {$[65]$} \\
\hline mir-503 & $\begin{array}{l}\text { hsa-miR-503-5p } \\
\text { (hsa-miR-503) }\end{array}$ & URS00000F6E49_9606 & UAGCAGCGGGAACAGUUCUGCAG & {$[65]$} \\
\hline \multirow[t]{2}{*}{ mir-505 } & $\begin{array}{l}\text { hsa-miR-505-3p } \\
\text { (hsa-miR-505) }\end{array}$ & URS00004A5A07_9606 & CGUCAACACUUGCUGGUUUCCU & {$[65]$} \\
\hline & $\begin{array}{l}\text { hsa-miR-505-5p } \\
\text { (hsa-miR-505*) }\end{array}$ & URS000017EA6A_9606 & GGGAGCCAGGAAGUAUUGAUGU & {$[65]$} \\
\hline \multirow[t]{4}{*}{ mir-506 } & $\begin{array}{l}\text { hsa-miR-508-3p } \\
\text { (hsa-miR-508) }\end{array}$ & URS000044FE6A_9606 & UGAUUGUAGCCUUUUGGAGUAGA & {$[65]$} \\
\hline & hsa-miR-512-3p & URS000020F110_9606 & AAGUGCUGUCAUAGCUGAGGUC & {$[65]$} \\
\hline & hsa-miR-512-5p & URS0000062B37_9606 & CACUCAGCCUUGAGGGCACUUUC & {$[65]$} \\
\hline & $\begin{array}{l}\text { hsa-miR-513a-5p } \\
\text { (hsa-miR-513-5p) }\end{array}$ & URS0000357286_9606 & UUCACAGGGAGGUGUCAU & {$[65]$} \\
\hline \multirow[t]{13}{*}{ mir-515 } & $\begin{array}{l}\text { hsa-miR-517c-3p } \\
\text { (hsa-miR-517c) }\end{array}$ & $\begin{array}{l}\text { URS00003FBECA_ } \\
9606\end{array}$ & AUCGUGCAUCCUUUUAGAGUGU & {$[65]$} \\
\hline & hsa-miR-520c-3p & URS000049A7EB_9606 & AAAGUGCUUCCUUUUAGAGGGU & {$[65]$} \\
\hline & hsa-miR-515-5p & URS00000A68B2_9606 & UUCUCCAAAAGAAAGCACUUUCUG & {$[65]$} \\
\hline & $\begin{array}{l}\text { hsa-miR-516b-3p } \\
\text { (hsa-miR-516-3p) }\end{array}$ & - & UGCUUCCUUUCAGAGGGU & [65] \\
\hline & $\begin{array}{l}\text { hsa-miR-517a-3p } \\
\text { (hsa-miR-517a) }\end{array}$ & URS00000D4AB5_9606 & AUCGUGCAUCCCUUUAGAGUGU & {$[65]$} \\
\hline & hsa-miR-518a-3p & URS0000024ACC_9606 & GAAAGCGCUUCCCUUUGCUGGA & {$[65]$} \\
\hline & hsa-miR-518b & URS00003676C9_9606 & CAAAGCGCUCCCCUUUAGAGGU & {$[65]$} \\
\hline & $\begin{array}{l}\text { hsa-miR-518d-3p } \\
\text { (hsa-miR-518d) }\end{array}$ & URS00001B6361_9606 & CAAAGCGCUUCCCUUUGGAGC & {$[65]$} \\
\hline & $\begin{array}{l}\text { hsa-miR-518f-3p } \\
\text { (hsa-miR-518f) }\end{array}$ & URS000075E9BD_9606 & GAAAGCGCUUCUCUUUAGAGG & {$[65]$} \\
\hline & $\begin{array}{l}\text { hsa-miR-519a-3p } \\
\text { (hsa-miR-519a) }\end{array}$ & URS0000135E29_9606 & AAAGUGCAUCCUUUUAGAGUGU & {$[65]$} \\
\hline & hsa-miR-519b-3p & URS00003883FE_9606 & AAAGUGCAUCCUUUUAGAGGUU & {$[65]$} \\
\hline & $\begin{array}{l}\text { hsa-miR-519e-5p } \\
\text { (hsa-miR-519e*) }\end{array}$ & URS000075AC86_9606 & UUCUCCAAAAGGGAGCACUUUC & {$[65]$} \\
\hline & $\begin{array}{l}\text { hsa-miR-520a-3p } \\
\text { (hsa-miR-520a) }\end{array}$ & URS0000101689_9606 & AAAGUGCUUCCCUUUGGACUGU & {$[65]$} \\
\hline \multirow[t]{2}{*}{ mir-541 } & $\begin{array}{l}\text { hsa-miR-541-3p } \\
\text { (hsa-miR-541) }\end{array}$ & URS000075A3AC_9606 & UGGUGGGCACAGAAUCUGGACU & {$[65]$} \\
\hline & $\begin{array}{l}\text { hsa-miR-541-5p } \\
\text { (hsa-miR-541*) }\end{array}$ & URS0000076E54_9606 & AAAGGAUUCUGCUGUCGGUCCCACU & {$[65]$} \\
\hline \multirow[t]{2}{*}{ mir-542 } & hsa-miR-542-3p & URS00004F859B_9606 & UGUGACAGAUUGAUAACUGAAA & {$[53,64,65]$} \\
\hline & hsa-miR-542-5p & URS000050C722_9606 & UCGGGGAUCAUCAUGUCACGAGA & [65] \\
\hline \multirow[t]{8}{*}{$\operatorname{mir}-548$} & $\begin{array}{l}\text { hsa-miR-548a-3p } \\
\text { (hsa-miR-548a) }\end{array}$ & URS000038037E_9606 & CAAAACUGGCAAUUACUUUUGC & {$[65]$} \\
\hline & $\begin{array}{l}\text { hsa-miR-548aa\# } \\
\text { hsa-miR-548t-3p }\end{array}$ & URS000012930C_9606 & AAAAACCACAAUUACUUUUGCACCA & {$[66]$} \\
\hline & hsa-miR-548ap-5p & URS000054B69F_9606 & AAAAGUAAUUGCGGUCUUU & [73] \\
\hline & $\begin{array}{l}\text { hsa-miR-548b-3p } \\
\text { (hsa-miR-548b) }\end{array}$ & URS000039A25B_9606 & CAAGAACCUCAGUUGCUUUUGU & {$[65]$} \\
\hline & $\begin{array}{l}\text { hsa-miR-548c-3p } \\
\text { (hsa-miR-548c) }\end{array}$ & URS0000614A9B_9606 & CAAAAAUCUCAAUUACUUUUGC & {$[65]$} \\
\hline & hsa-miR-548d-5p & URS00005F2D64_9606 & AAAAGUAAUUGUGGUUUUUGCC & {$[65]$} \\
\hline & $\begin{array}{l}\text { hsa-miR-570-3p } \\
\text { (hsa-miR-570) }\end{array}$ & URS0000250A40_9606 & CGAAAACAGCAAUUACCUUUGC & {$[65]$} \\
\hline & hsa-miR-603 & URS000075A6F1_9606 & CACACACUGCAAUUACUUUUGC & {$[65]$} \\
\hline mir-549 & hsa-miR-549a-3p & URS00004C689A_9606 & UGACAACUAUGGAUGAGCUCU & {$[65]$} \\
\hline
\end{tabular}


Table 2 (continued)

\begin{tabular}{|c|c|c|c|c|}
\hline Family & Name & RNAcental & Sequence & Ref. \\
\hline $\operatorname{mir}-550$ & $\begin{array}{l}\text { (hsa-miR-549) } \\
\text { hsa-miR-550a-5p } \\
\text { (hsa-miR-550) }\end{array}$ & URS00003FFA6C_9606 & AGUGCCUGAGGGAGUAAGAGCCC & {$[65]$} \\
\hline $\operatorname{mir}-551$ & hsa-miR-551a & URS00002E99CB_9606 & GCGACCCACUCUUGGUUUCCA & {$[65]$} \\
\hline $\operatorname{mir}-556$ & hsa-miR-556-3p & URS00001D6605_9606 & AUAUUACCAUUAGCUCAUCUUU & {$[65]$} \\
\hline mir-561 & $\begin{array}{l}\text { hsa-miR-561-3p } \\
\text { (hsa-miR-561) }\end{array}$ & URS000075D1DD_9606 & CAAAGUUUAAGAUCCUUGAAGU & {$[65]$} \\
\hline mir-564 & hsa-miR-564 & URS000075ED17_9606 & AGGCACGGUGUCAGCAGGC & {$[65]$} \\
\hline mir-571 & hsa-miR-571 & URS000075C61C_9606 & UGAGUUGGCCAUCUGAGUGAG & {$[65]$} \\
\hline mir-572 & hsa-miR-572 & URS000075CEB8_9606 & GUCCGCUCGGCGGUGGCCCA & {$[65]$} \\
\hline \multirow[t]{2}{*}{ mir-574 } & hsa-miR-574-3p & URS00001CF056_9606 & CACGCUCAUGCACACACCCACA & {$[65,66,72]$} \\
\hline & hsa-miR-574-5p & URS000057466C_9606 & UGAGUGUGUGUGUGUGAGUGUGU & {$[66,72]$} \\
\hline $\operatorname{mir}-582$ & hsa-miR-582-3p & URS00002573C3_9606 & UAACUGGUUGAACAACUGAACC & {$[65]$} \\
\hline mir-584 & $\begin{array}{l}\text { hsa-miR-584-5p } \\
\text { (hsa-miR-584) }\end{array}$ & URS0000576F83_9606 & UUAUGGUUUGCCUGGGACUGAG & {$[65]$} \\
\hline $\operatorname{mir}-589$ & $\begin{array}{l}\text { hsa-miR-589-5p } \\
\text { (hsa-miR-589) }\end{array}$ & URS00004214BB_9606 & UGAGAACCACGUCUGCUCUGAG & {$[65]$} \\
\hline \multirow[t]{2}{*}{$\operatorname{mir}-590$} & hsa-miR-590-3P & URS0000272039_9606 & UAAUUUUAUGUAUAAGCUAGU & {$[65]$} \\
\hline & hsa-miR-590-5p & $\begin{array}{l}\text { URS00005CACA0_ } \\
9606\end{array}$ & GAGCUUAUUCAUAAAAGUGCAG & {$[65,70]$} \\
\hline $\operatorname{mir}-592$ & hsa-miR-592 & URS00004F507C_9606 & UUGUGUCAAUAUGCGAUGAUGU & {$[65]$} \\
\hline mir-593 & $\begin{array}{l}\text { hsa-miR-593-3p } \\
\text { (hsa-miR-593) }\end{array}$ & URS000075D407_9606 & UGUCUCUGCUGGGGUUUCU & {$[65]$} \\
\hline $\operatorname{mir}-595$ & hsa-miR-595 & URS000075B75E_9606 & GAAGUGUGCCGUGGUGUGUCU & {$[65]$} \\
\hline mir-596 & hsa-miR-596 & URS000075B35F_9606 & AAGCCUGCCCGGCUCCUCGGG & {$[65]$} \\
\hline $\operatorname{mir}-6089$ & hsa-miR-6089 & URS000075B63F_9606 & GGAGGCCGGGGUGGGGCGGGGCGG & {$[12]$} \\
\hline \multirow[t]{2}{*}{$\operatorname{mir}-615$} & hsa-miR-615-3p & URS00003D5391_9606 & UCCGAGCCUGGGUCUCCCUCUU & {$[53,54]$} \\
\hline & hsa-miR-615-5p & URS00004D8280_9606 & GGGGGUCCCCGGUGCUCGGAUC & {$[65,67]$} \\
\hline $\operatorname{mir}-616$ & $\begin{array}{l}\text { hsa-miR-616-3p } \\
\text { (hsa-miR-616) }\end{array}$ & URS00005E3F32_9606 & AGUCAUUGGAGGGUUUGAGCAG & {$[65]$} \\
\hline $\operatorname{mir}-618$ & hsa-miR-618 & URS0000450F92_9606 & AAACUCUACUUGUCCUUCUGAGU & {$[65]$} \\
\hline mir-619 & hsa-miR-619-5p & URS000075B584_9606 & GCUGGGAUUACAGGCAUGAGCC & {$[54,66]$} \\
\hline $\operatorname{mir}-622$ & hsa-miR-622 & URS000075E944_9606 & ACAGUCUGCUGAGGUUGGAGC & {$[65]$} \\
\hline $\operatorname{mir}-623$ & hsa-miR-623 & URS000075DCB1_9606 & AUCCCUUGCAGGGGCUGUUGGGU & {$[65]$} \\
\hline mir-625 & $\begin{array}{l}\text { hsa-miR-625-3p } \\
\text { (hsa-miR-625*) }\end{array}$ & URS0000475E09_9606 & GACUAUAGAACUUUCCCCCUCA & {$[65]$} \\
\hline $\operatorname{mir}-628$ & hsa-miR-628-3p & URS000061BE3B_9606 & UCUAGUAAGAGUGGCAGUCGA & {$[65]$} \\
\hline $\operatorname{mir}-629$ & $\begin{array}{l}\text { hsa-miR-629-5p } \\
\text { (hsa-miR-629) }\end{array}$ & URS00002F3336_9606 & UGGGUUUACGUUGGGAGAACU & {$[65]$} \\
\hline mir-636 & hsa-miR-636 & URS000075A79D_9606 & UGUGCUUGCUCGUCCCGCCCGCA & {$[65]$} \\
\hline mir-638 & hsa-miR-638 & URS000075DB2F_9606 & AGGGAUCGCGGGCGGGUGGCGGCCU & {$[12,65,70,72]$} \\
\hline mir-639 & hsa-miR-639 & URS000075B8B8_9606 & AUCGCUGCGGUUGCGAGCGCUGU & {$[65]$} \\
\hline mir-641 & hsa-miR-641 & URS000039D790_9606 & AAAGACAUAGGAUAGAGUCACCUC & {$[65]$} \\
\hline \multirow[t]{2}{*}{$\operatorname{mir}-642$} & $\begin{array}{l}\text { hsa-miR-642a-5p } \\
\text { (hsa-miR-642) }\end{array}$ & URS00000F2C33_9606 & GUCCCUCUCCAAAUGUGUCUUG & {$[65]$} \\
\hline & hsa-miR-642b-5p & URS000075B1CE_9606 & GGUUCCCUCUCCAAAUGUGUCU & {$[73]$} \\
\hline mir-649 & hsa-miR-649 & URS000075DD5B_9606 & AAACCUGUGUUGUUCAAGAGUC & {$[65]$} \\
\hline $\operatorname{mir}-650$ & hsa-miR-650 & URS000075A00C_9606 & AGGAGGCAGCGCUCUCAGGAC & {$[65]$} \\
\hline \multirow[t]{2}{*}{$\operatorname{mir}-6511$} & hsa-miR-6511a-5p & URS000075C82B_9606 & CAGGCAGAAGUGGGGCUGACAGG & {$[67]$} \\
\hline & hsa-miR-6511b-3p & URS0000759CCE_9606 & CCUCACCACCCCUUCUGCCUGCA & {$[67]$} \\
\hline mir-652 & $\begin{array}{l}\text { hsa-miR-652-3p } \\
\text { (hsa-miR-652) }\end{array}$ & URS0000013DD8_9606 & AAUGGCGCCACUAGGGUUGUG & {$[64]$} \\
\hline \multirow[t]{2}{*}{ mir-654 } & hsa-miR-654-3p & URS00002F40E9_9606 & UAUGUCUGCUGACCAUCACCUU & {$[65]$} \\
\hline & hsa-miR-654-5p & URS00002B0B46_9606 & UGGUGGGCCGCAGAACAUGUGC & {$[65]$} \\
\hline
\end{tabular}


Table 2 (continued)

\begin{tabular}{|c|c|c|c|c|}
\hline Family & Name & RNAcental & Sequence & Ref. \\
\hline & (hsa-miR-654) & & & \\
\hline $\operatorname{mir}-657$ & hsa-miR-657 & URS000075C4C7_9606 & GGCAGGUUCUCACCCUCUCUAGG & {$[65]$} \\
\hline $\operatorname{mir}-661$ & hsa-miR-661 & URS000075A4E8_9606 & UGCCUGGGUCUCUGGCCUGCGCGU & {$[65]$} \\
\hline \multirow[t]{2}{*}{ mir-663 } & hsa-miR-663a & URS00004929F1_9606 & AGGCGGGGCGCCGCGGGACCGC & {$[54,66,67]$} \\
\hline & hsa-miR-663b & URS000075C3F6_9606 & GGUGGCCCGGCCGUGCCUGAGG & {$[54,65,67]$} \\
\hline mir-664 & $\begin{array}{l}\text { hsa-miR-664a-3p } \\
\text { (hsa-miR-664) [65] }\end{array}$ & URS000029AE45_9606 & UAUUCAUUUAUCCCCAGCCUACA & {$[65,66]$} \\
\hline $\operatorname{mir}-665$ & hsa-miR-665 & URS0000355E82_9606 & ACCAGGAGGCUGAGGCCCCU & {$[65,67]$} \\
\hline \multirow[t]{2}{*}{ mir-671 } & hsa-miR-671-3p & URS00002B7450_9606 & UCCGGUUCUCAGGGCUCCACC & {$[65,67]$} \\
\hline & hsa-miR-671-5p & URS00002FB368_9606 & AGGAAGCCCUGGAGGGGCUGGAG & {$[67]$} \\
\hline $\operatorname{mir}-6724$ & hsa-miR-6724-5p & URS00007777B8_9606 & CUGGGCCCGCGGCGGGCGUGGGG & {$[67]$} \\
\hline $\operatorname{mir}-675$ & hsa-miR-675-5p & URS00004E5112_9606 & UGGUGCGGAGAGGGCCCACAGUG & {$[67]$} \\
\hline mir-7 & $\begin{array}{l}\text { hsa-miR-7-2-3p } \\
\text { (hsa-miR-7-2*) }\end{array}$ & URS0000572E11_9606 & CAACAAAUCCCAGUCUACCUAA & {$[65]$} \\
\hline mir-708 & $\begin{array}{l}\text { hsa-miR-708-5p } \\
\text { (hsa-miR-708) }\end{array}$ & URS000019D79B_9606 & AAGGAGCUUACAAUCUAGCUGGG & {$[65]$} \\
\hline \multirow[t]{2}{*}{ mir-743 } & $\begin{array}{l}\text { hsa-miR-888-5p } \\
\text { (hsa-miR-888) }\end{array}$ & URS000075D73F_9606 & UACUCAAAAAGCUGUCAGUCA & {$[65]$} \\
\hline & hsa-miR-892b & URS000075A42A_9606 & CACUGGCUCCUUUCUGGGUAGA & {$[65]$} \\
\hline \multirow[t]{2}{*}{ mir-744 } & $\begin{array}{l}\text { hsa-miR-744-3p } \\
\text { (hsa-miR-744*) }\end{array}$ & URS00005FAA14_9606 & CUGUUGCCACUAACCUCAACCU & {$[65]$} \\
\hline & $\begin{array}{l}\text { hsa-miR-744-5p } \\
\text { (hsa-miR-744) }\end{array}$ & URS00002ED61F_9606 & UGCGGGGCUAGGGCUAACAGCA & {$[65]$} \\
\hline $\operatorname{mir}-760$ & hsa-miR-760 & URS0000512C88_9606 & CGGCUCUGGGUCUGUGGGGA & {$[67]$} \\
\hline mir-762 & hsa-miR-762 & URS0000327AFF_9606 & GGGGCUGGGGCCGGGGCCGAGC & {$[72]$} \\
\hline $\operatorname{mir}-7641$ & hsa-miR-7641 & URS000075B793_9606 & UUGAUCUCGGAAGCUAAGC & {$[54,66]$} \\
\hline $\operatorname{mir}-766$ & $\begin{array}{l}\text { hsa-miR-766-3p } \\
\text { (hsa-miR-766) }\end{array}$ & URS00001012BC_9606 & ACUCCAGCCCCACAGCCUCAGC & {$[65]$} \\
\hline mir-769 & hsa-miR-769-5p & URS00004E008F_9606 & UGAGACCUCUGGGUUCUGAGCU & {$[54,65]$} \\
\hline $\operatorname{mir}-770$ & hsa-miR-770-5p & URS000075A169_9606 & UCCAGUACCACGUGUCAGGGCCA & {$[65]$} \\
\hline \multirow[t]{6}{*}{$\operatorname{mir}-8$} & $\begin{array}{l}\text { hsa-miR-141-3p } \\
\text { (hsa-miR-141) }\end{array}$ & URS000003E1A9_9606 & UAACACUGUCUGGUAAAGAUGG & {$[65]$} \\
\hline & $\begin{array}{l}\text { hsa-miR-200a-3p } \\
\text { (hsa-miR-200a) }\end{array}$ & URS000008DA94_9606 & UAACACUGUCUGGUAACGAUGU & {$[65]$} \\
\hline & $\begin{array}{l}\text { hsa-miR-200a-5p } \\
\text { (hsa-miR-200a*) }\end{array}$ & URS000023B77E_9606 & CAUCUUACCGGACAGUGCUGGA & {$[65]$} \\
\hline & $\begin{array}{l}\text { hsa-miR-200b-3p } \\
\text { (hsa-miR-200b) }\end{array}$ & URS000014D9C1_9606 & UAAUACUGCCUGGUAAUGAUGA & {$[65]$} \\
\hline & $\begin{array}{l}\text { hsa-miR-200c-3p } \\
\text { (hsa-miR-200c) }\end{array}$ & URS0000192F9C_9606 & UAAUACUGCCGGGUAAUGAUGGA & {$[64,65]$} \\
\hline & hsa-miR-429 & URS000055BBE5_9606 & UAAUACUGUCUGGUAAAACCGU & {$[65]$} \\
\hline $\operatorname{mir}-8069$ & hsa-miR-8069 & URS000075E1C1_9606 & GGAUGGUUGGGGGCGGUCGGCGU & {$[12]$} \\
\hline $\operatorname{mir}-874$ & hsa-miR-874-3p & URS00005609ED_9606 & CUGCCCUGGCCCGAGGGACCGA & {$[67]$} \\
\hline $\operatorname{mir}-875$ & hsa-miR-875-5p & URS0000312ECD_9606 & UAUACCUCAGUUUUAUCAGGUG & {$[65]$} \\
\hline mir-876 & hsa-miR-876-5p & URS0000470305_9606 & UGGAUUUCUUUGUGAAUCACCA & {$[65]$} \\
\hline mir-885 & hsa-miR-885-5p & URS0000246356_9606 & UCCAUUACACUACCCUGCCUCU & {$[65]$} \\
\hline \multirow[t]{2}{*}{ mir-9 } & $\begin{array}{l}\text { hsa-miR-9-3p } \\
\text { (hsa-miR-9*) }\end{array}$ & URS00003496BE_9606 & AUAAAGCUAGAUAACCGAAAGU & {$[65]$} \\
\hline & $\begin{array}{l}\text { hsa-miR-9-5p } \\
\text { (hsa-miR-9) [65] }\end{array}$ & URS00004208C5_9606 & UCUUUGGUUAUCUAGCUGUAUGA & {$[54,65]$} \\
\hline miR-922 & hsa-miR-922 & URS000075D35F_9606 & GCAGCAGAGAAUAGGACUACGUC & {$[65]$} \\
\hline miR-935 & hsa-miR-935 & URS000033EBB8_9606 & CCAGUUACCGCUUCCGCUACCGC & {$[65]$} \\
\hline mir-937 & $\begin{array}{l}\text { hsa-miR-937-3p } \\
\text { (hsa-miR-937) }\end{array}$ & URS0000553F51_9606 & AUCCGCGCUCUGACUCUCUGCC & {$[65]$} \\
\hline mir-938 & hsa-miR-938 & URS000075DF80_9606 & UGCCCUUAAAGGUGAACCCAGU & {$[65]$} \\
\hline
\end{tabular}


Table 2 (continued)

\begin{tabular}{|c|c|c|c|c|}
\hline Family & Name & RNAcental & Sequence & Ref. \\
\hline mir-939 & $\begin{array}{l}\text { hsa-miR-939-5p } \\
\text { (hsa-miR-939) }\end{array}$ & URS00005A31EB_9606 & UGGGGAGCUGAGGCUCUGGGGGUG & {$[65]$} \\
\hline mir-941 & hsa-miR-941 & URS000050E4BA_9606 & CACCCGGCUGUGUGCACAUGUGC & {$[65]$} \\
\hline \multirow[t]{45}{*}{$\operatorname{mir}-95$} & $\begin{array}{l}\text { hsa-miR-545-3p } \\
\text { (hsa-miR-545) }\end{array}$ & URS00002E1509_9606 & UCAGCAAACAUUUAUUGUGUGC & {$[65]$} \\
\hline & $\begin{array}{l}\text { hsa-miR-545-5p } \\
\text { (hsa-miR-545*) }\end{array}$ & URS00004C4520_9606 & UCAGUAAAUGUUUAUUAGAUGA & {$[65]$} \\
\hline & hsa-let-7c & - & - & {$[65]$} \\
\hline & hsa-miR-1 & - & - & {$[65]$} \\
\hline & hsa-miR-10 & URS00005D8C46_9606 & UACCCUGUAGAACCGAAUUUG & {$[74]$} \\
\hline & hsa-miR-10395-3p & URS0000D52042_9606 & AUGUAUUCGUACUGUCUGAUG & {$[59]$} \\
\hline & hsa-miR-10395-5p & URS0000D53F1E_9606 & GUGAUGGAGAGCAAUACC & {$[59]$} \\
\hline & hsa-miR-1180 & - & - & {$[65]$} \\
\hline & hsa-miR-1234-5p & - & - & {$[72]$} \\
\hline & hsa-miR-1274a & - & - & {$[65]$} \\
\hline & hsa-miR-1274b & - & - & {$[65]$} \\
\hline & hsa-miR-1298 & - & - & {$[65]$} \\
\hline & hsa-miR-1300 & - & - & {$[65]$} \\
\hline & hsa-miR-133a & - & - & {$[65]$} \\
\hline & hsa-miR-152 & - & - & {$[65]$} \\
\hline & hsa-miR-190b & - & - & {$[65]$} \\
\hline & hsa-miR-199 & URS000027FB26_9606 & CCCAGUGUUUAGACUAUCUGU & {$[74]$} \\
\hline & hsa-miR-210 & - & - & {$[65]$} \\
\hline & hsa-miR-215 & - & - & {$[65]$} \\
\hline & hsa-miR-219-2-3p & - & - & {$[65]$} \\
\hline & hsa-miR-2277-5p & URS00000D6C3F_9606 & AGCGCGGGCUGAGCGCUGCCAGUC & {$[67]$} \\
\hline & hsa-miR-23-3p & - & - & {$[73]$} \\
\hline & hsa-miR-26 & - & - & {$[74]$} \\
\hline & hsa-miR-3178 & URS0000365675_9606 & GGGGCGCGGCCGGAUCG & {$[12]$} \\
\hline & hsa-miR-3195 & URS000004DB7E_9606 & CGCGCCGGGCCCGGGUU & {$[54]$} \\
\hline & hsa-miR-3196 & URS000033B548_9606 & CGGGGCGGCAGGGGCCUC & {$[12]$} \\
\hline & hsa-miR-328 & - & - & {$[65]$} \\
\hline & hsa-miR-329 & - & - & {$[64,65]$} \\
\hline & hsa-miR-3614-5p & URS00003D4175_9606 & CCACUUGGAUCUGAAGGCUGCCC & {$[54]$} \\
\hline & hsa-miR-3653-3p & URS000009AF54_9606 & CUAAGAAGUUGACUGAAG & {$[54]$} \\
\hline & hsa-miR-3656 & URS0000514CEC_9606 & GGCGGGUGCGGGGGUGG & {$[12,72]$} \\
\hline & hsa-miR-3665 & URS000075AFFF_9606 & AGCAGGUGCGGGGCGGCG & {$[12]$} \\
\hline & hsa-miR-370 & - & - & {$[65]$} \\
\hline & hsa-miR-375 & - & - & {$[65]$} \\
\hline & hsa-miR-378c & URS000025307A_9606 & ACUGGACUUGGAGUCAGAAGAGUGG & {$[54]$} \\
\hline & hsa-miR-383 & - & - & {$[65]$} \\
\hline & hsa-miR-3944-3p & URS0000446855_9606 & UUCGGGCUGGCCUGCUGCUCCGG & {$[67]$} \\
\hline & hsa-miR-410 & - & - & {$[65]$} \\
\hline & hsa-miR-412 & - & - & {$[65]$} \\
\hline & hsa-miR-4284 & URS00001FC26E_9606 & GGGCUCACAUCACCCCAU & {$[72]$} \\
\hline & hsa-miR-433 & - & - & {$[65]$} \\
\hline & hsa-miR-4443 & URS00004D84DB_9606 & UUGGAGGCGUGGGUUUU & {$[72]$} \\
\hline & hsa-miR-4448 & URS00005F305A_9606 & GGCUCCUUGGUCUAGGGGUA & {$[54]$} \\
\hline & hsa-miR-4454 & URS00005D12AC_9606 & GGAUCCGAGUCACGGCACCA & {$[12,54,66]$} \\
\hline & hsa-miR-4461 & URS000028425A_9606 & GAUUGAGACUAGUAGGGCUAGGC & {$[54]$} \\
\hline
\end{tabular}


Table 2 (continued)

\begin{tabular}{|c|c|c|c|c|}
\hline Family & Name & RNAcental & Sequence & Ref. \\
\hline & hsa-miR-4466 & URS00001DC1D3_9606 & GGGUGCGGGCCGGCGGGG & {$[12,54,72]$} \\
\hline & $\begin{array}{l}\text { hsa-miR-4485-3p } \\
\text { (hsa-miR-4485) }\end{array}$ & URS000038446A_9606 & UAACGGCCGCGGUACCCUAA & {$[11]$} \\
\hline & hsa-miR-4492 & URS000045ED38_9606 & GGGGCUGGGCGCGCGCC & {$[54]$} \\
\hline & hsa-miR-4497 & URS00000A2C49_9606 & CUCCGGGACGGCUGGGC & [12] \\
\hline & hsa-miR-4505 & URS000075EBEE_9606 & AGGCUGGGCUGGGACGGA & [72] \\
\hline & hsa-miR-4508 & URS00004E78D3_9606 & GCGGGGCUGGGCGCGCG & {$[12,54]$} \\
\hline & hsa-miR-4516 & URS00000BF7F9_9606 & GGGAGAAGGGUCGGGGC & {$[12,54,66]$} \\
\hline & hsa-miR-4532 & URS000013A349_9606 & CCCCGGGGAGCCCGGCG & {$[54,66,67]$} \\
\hline & hsa-miR-4649-5p & URS000044FB51_9606 & UGGGCGAGGGGUGGGCUCUCAGAG & {$[67]$} \\
\hline & hsa-miR-4665-5p & URS00000E9F44_9606 & CUGGGGGACGCGUGAGCGCGAGC & [67] \\
\hline & hsa-miR-4668-5p & URS00000A17E7_9606 & AGGGAAAAAAAAAAGGAUUUGUC & [12] \\
\hline & hsa-miR-4687-3p & URS000047456A_9606 & UGGCUGUUGGAGGGGGCAGGC & {$[72]$} \\
\hline & hsa-miR-4707-5p & URS00003EB443_9606 & GCCCCGGCGCGGGCGGGUUCUGG & [67] \\
\hline & hsa-miR-4708-3p & URS00004F4FFB_9606 & AGCAAGGCGGCAUCUCUCUGAU & [73] \\
\hline & hsa-miR-4722-5p & URS000047996E_9606 & GGCAGGAGGGCUGUGCCAGGUUG & [67] \\
\hline & hsa-miR-4741 & URS0000547F6A_9606 & CGGGCUGUCCGGAGGGGUCGGCU & [67] \\
\hline & hsa-miR-4763-3p & URS00004A40D8_9606 & AGGCAGGGGCUGGUGCUGGGCGGG & {$[67,72]$} \\
\hline & hsa-miR-4787-5p & URS0000521832_9606 & GCGGGGGUGGCGGCGGCAUCCC & {$[12,54,72]$} \\
\hline & hsa-miR-4792 & URS00005B6542_9606 & CGGUGAGCGCUCGCUGGC & {$[54,66]$} \\
\hline & hsa-miR-487a & - & - & {$[65]$} \\
\hline & hsa-miR-487b & - & - & {$[65]$} \\
\hline & hsa-miR-489 & - & - & {$[65]$} \\
\hline & hsa-miR-494 & - & - & {$[65]$} \\
\hline & hsa-miR-5088-5p & URS00002F0130_9606 & CAGGGCUCAGGGAUUGGAUGGAGG & [67] \\
\hline & hsa-miR-5095 & URS00002E1785_9606 & UUACAGGCGUGAACCACCGCG & {$[54]$} \\
\hline & hsa-miR-5096 & URS00001F8B82_9606 & GUUUCACCAUGUUGGUCAGGC & {$[54,66]$} \\
\hline & hsa-miR-5100 & URS0000079F78_9606 & UUCAGAUCCCAGCGGUGCCUCU & {$[12]$} \\
\hline & hsa-miR-5191 & URS000075CB1C_9606 & AGGAUAGGAAGAAUGAAGUGCU & {$[54]$} \\
\hline & hsa-miR-520b & - & - & {$[65]$} \\
\hline & hsa-miR-520f & - & - & {$[65]$} \\
\hline & hsa-miR-520g & - & - & {$[65]$} \\
\hline & hsa-miR-5585-3p & URS00003E6EFA_9606 & CUGAAUAGCUGGGACUACAGGU & {$[54,66]$} \\
\hline & hsa-miR-566 & URS00000FD5FE_9606 & GGGCGCCUGUGAUCCCAAC & {$[65]$} \\
\hline & hsa-miR-5787 & URS000075CA3A_9606 & GGGCUGGGGCGCGGGGAGGU & {$[12,72]$} \\
\hline & hsa-miR-597 & - & - & {$[65]$} \\
\hline & hsa-miR-598 & - & - & {$[65]$} \\
\hline & hsa-miR-605 & - & - & {$[65]$} \\
\hline & hsa-miR-6068 & URS000075E142_9606 & CCUGCGAGUCUCCGGCGGUGG & [72] \\
\hline & hsa-miR-6087 & URS000075EF8B_9606 & UGAGGCGGGGGGGCGAGC & {$[12,54,66,67]$} \\
\hline & hsa-miR-6088 & URS000075EC34_9606 & AGAGAUGAAGCGGGGGGGCG & {$[12,72]$} \\
\hline & hsa-miR-6090 & URS0000759F58_9606 & GGGGAGCGAGGGGCGGGGC & [12] \\
\hline & hsa-miR-6124 & URS000075CC26_9606 & GGGAAAAGGAAGGGGGAGGA & {$[72]$} \\
\hline & hsa-miR-6125 & URS000075F0F0_9606 & GCGGAAGGCGGAGCGGCGGA & {$[12]$} \\
\hline & hsa-miR-6126 & URS000075D118_9606 & GUGAAGGCCCGGCGGAGA & {$[66]$} \\
\hline & hsa-miR-627 & - & - & {$[65]$} \\
\hline & hsa-miR-655 & - & - & {$[65]$} \\
\hline & hsa-miR-656 & - & - & {$[65]$} \\
\hline & hsa-miR-659-3p & URS000075C04A_9606 & CUUGGUUCAGGGAGGGUCCCCA & {$[65]$} \\
\hline
\end{tabular}


Table 2 (continued)

\begin{tabular}{|c|c|c|c|c|}
\hline Family & Name & RNAcental & Sequence & Ref. \\
\hline & \multicolumn{4}{|l|}{ (hsa-miR-659) } \\
\hline & hsa-miR-668 & - & - & {$[65]$} \\
\hline & hsa-miR-672 & - & - & {$[65]$} \\
\hline & hsa-miR-6727-5p & URS000075A9AA_9606 & CUCGGGGCAGGCGGCUGGGAGCG & {$[12,67]$} \\
\hline & hsa-miR-6729-5p & URS000075DD20_9606 & UGGGCGAGGGCGGCUGAGCGGC & {$[12,67]$} \\
\hline & hsa-miR-6739-5p & URS000075C51C_9606 & UGGGAAAGAGAAAGAACAAGUA & {$[66]$} \\
\hline & hsa-miR-6746-5p & URS000075AF8F_9606 & CCGGGAGAAGGAGGUGGCCUGG & {$[67]$} \\
\hline & hsa-miR-6789-5p & URS000075DD04_9606 & GUAGGGGCGUCCCGGGCGCGCGGG & [67] \\
\hline & hsa-miR-6821-5p & URS000075EAF3_9606 & GUGCGUGGUGGCUCGAGGCGGGG & {$[67]$} \\
\hline & hsa-miR-6858-5p & URS000075C360_9606 & GUGAGGAGGGGCUGGCAGGGAC & {$[67]$} \\
\hline & hsa-miR-6869-5p & URS000075C3FC_9606 & GUGAGUAGUGGCGCGCGGCGGC & {$[12]$} \\
\hline & hsa-miR-6891-5p & URS000075BD73_9606 & UAAGGAGGGGGAUGAGGGG & [67] \\
\hline & hsa-miR-720 & - & - & {$[65]$} \\
\hline & hsa-miR-7704 & URS000028F729_9606 & CGGGGUCGGCGGCGACGUG & {$[12,54,66]$} \\
\hline & hsa-miR-7977 & URS000075A1F7_9606 & UUCCCAGCCAACGCACCA & [12] \\
\hline & hsa-miR-8061 & URS000075E23B_9606 & CUUAGAUUAGAGGAUAUUGUU & {$[54]$} \\
\hline & hsa-miR-8485 & URS000076B539_9606 & CACACACACACACACACGUAU & {$[66]$} \\
\hline & hsa-miR-874 & - & - & {$[65]$} \\
\hline & hsa-miR-886-3p & - & - & {$[65]$} \\
\hline & hsa-miR-886-5p & - & - & {$[65]$} \\
\hline & hsa-miR-887 & - & - & [65] \\
\hline & hsa-miR-889 & - & - & {$[65]$} \\
\hline & hsa-miR-891a & - & - & {$[65]$} \\
\hline & hsa-miR-942 & - & - & {$[65]$} \\
\hline & hsa-miR-95 & - & - & {$[65]$} \\
\hline
\end{tabular}

\#\# The two names corresponded to the same sequence

\# Identified by the sequence and the precursor. The referred article uses a name not found in the databases

(hsa-miR-126-3p, hsa-miR-21-5p, hsa-miR-26a-5p, hsa-miR29b-3p), regulation of phosphatidylinositol 3-kinase signaling (hsa-miR-126-3p, hsa-miR-20a-5p, hsa-miR-21-5p), and positive regulation of cell migration (hsa-miR-1290, hsa-miR181b-5p, hsa-miR-21-5p, hsa-miR-29b-3p) (Table 4S). Therefore, they can also be implicated in the positive effects observed after the injection of human AT-MSC-EVs in animal model of osteoarthritis [66], and in osteoarthritis chondrocytes [66] and osteoblasts [78] in vitro.

Regarding the use of AT-MSC-EVs for cardiology and vascular diseases, the rationale may be the role of the detected miRNAs in negative regulation of heart rate (hsa-miR-26a$5 p$ ), regulation of heart contraction (hsa-miR-92a-3p), positive regulation of cardiac muscle cell proliferation (hsa-miR199b-3p, hsa-miR-19b-3p, hsa-miR-204-5p, hsa-miR-222$3 p$, hsa-miR-23b-3p), negative regulation of cardiac muscle cell apoptotic process (hsa-miR-145-5p, hsa-miR-199b-3p, hsa-miR-19b-3p, hsa-miR-21-5p, hsa-miR-30e-5p), regulation of cardiac muscle hypertrophy (hsa-miR-20a-5p), cell differentiation (hsa-miR-155-5p) and proliferation (hsa-miR199a-5p), and regulation of cardiac conduction (hsa-miR-19a$3 p$ ), among others (Table 4S). AT-MSC-EV proteins are also involved in some of these biological processes. Therefore, both types of molecules, proteins and miRNAS, may present a synergistic action, supporting the cardioprotection observed in an in vivo model of myocardial infarction after the administration of AT-MSC-EVs [79].

Numerous miRNAs are involved in the positive regulation of angiogenesis, such as hsa-miR-126-3p, hsa-miR-143-3p, hsa-miR-1908-5p, hsa-miR-199a-5p, hsa-miR-199b-3p, hsamiR-20a-5p, hsa-miR-21-5p, hsa-miR-27b-3p, hsa-miR-29a$3 p$ and hsa-miR-31-5p, among others (Table 4S). They may play a role in the promotion of angiogenesis, as observed both in vitro and in vivo $[60,72,80]$. However, it should be noted that there are also numerous miRNAs involved in the negative regulation of angiogenesis (see Table $4 \mathrm{~S}$ for a complete list).

Finally, although there are less miRNAs than proteins involved in regulation of cellular processes such as proliferation 
GO molecular function terms of miRNA

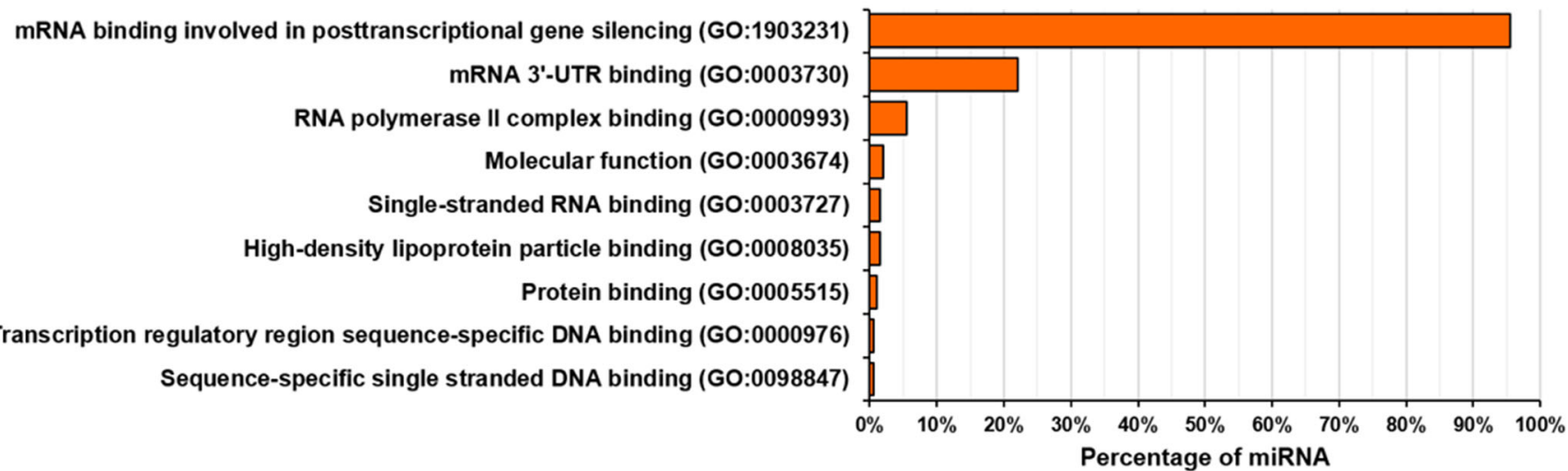

Fig. 5 Gene ontology (GO) molecular function terms of the miRNA detected in human AT-MSC-EVs. Only 199 miRNAs showed GO molecular function annotations. The $95 \%$ of them enables the mRNA binding involved in post-transcriptional gene silencing

and apoptosis (Tables $2 \mathrm{~S}$ and $4 \mathrm{~S}$ ), it should be noted that each miRNA targets more than one mRNA. Therefore, each one can show effects on numerous proteins.

\section{tRNA, mRNA, rRNA, snRNA, snoRNA and scRNA}

According to Kaur et al. [53], the detected tRNA in AT-MSCEVs represents $47 \%$ of all small RNAs observed. Although this percentage is slightly higher than that of miRNA, the available information about the presence of this type of RNA $[11,53,54]$ is significantly less. The main tRNAs, in order of quantity detected in AT-MSC-EVs, are tRNA GCC (Gly), tRNA CTC (Glu) and tRNA TTC (Glu). Surprisingly, in AT-MSC the tRNA CTC (Glu) is the most abundant, while tRNA GCC (Gly) makes up a significantly lower percentage than in AT-MSC-EVs [11]. Other tRNAs present in lesser amounts in AT-MSC-EVs are tRNA GTC (Asp), tRNA
CCC (Gly), tRNA GTG (His), tRNA CTT (Lys), tRNA AAC (Val) and tRNA CAC (Val) [11].

84 different mRNAs were detected in the AT-MSC-EVs. Their corresponding gene symbols, in order of quantity detected, are FN1, COL4A3, PGF, MMP2, PLG, HGF, IGF1, TEK, FGF2, HIF1A, VEGFA, EDN1, PF4, CXCL9, FGF1, TGFB2, ITGAV, PROK2, EGF, FLT1, IL8, IFNG, IFNA1, SERPINE1, FIGF, TIMP3, JAG1, CXCL10 ANGPT1, TIMP2, IL6, TIMP1, SERPINF1, AKT1, ANPEP, EFNB2, CXCL6, HPSE, THBS1, EPHB4, NRP1, THBS2, CCL11, TGFA, TIE1, TGFB1, COL18A1, PDGFA, KDR, F3, TGFBR1, BAI1, NRP2, ANGPT2, MMP9, CXCL1 ANGPTL4, ANG, ENG, PTGS1, CCL2, VEGFC, EFNA1, TNF, CTGF, NOS3, VEGFB, CXCL5, LECT1, CDH5, LEP, ITGB3, MMP14, IL1B, SPHK1, PLAU, FGFR3, ID1, S1PR1, ERBB2, PECAM1, NOTCH4, TYMP and MDK [52].
Fig. 6 Simplified outline of the molecular functions enables by the miRNA detected in human AT-MSC-EVs. For a complete review of the relationships between gene ontology terms see the chart view in the web-based tool QuickGO (https://www.ebi. ac.uk/QuickGO/)

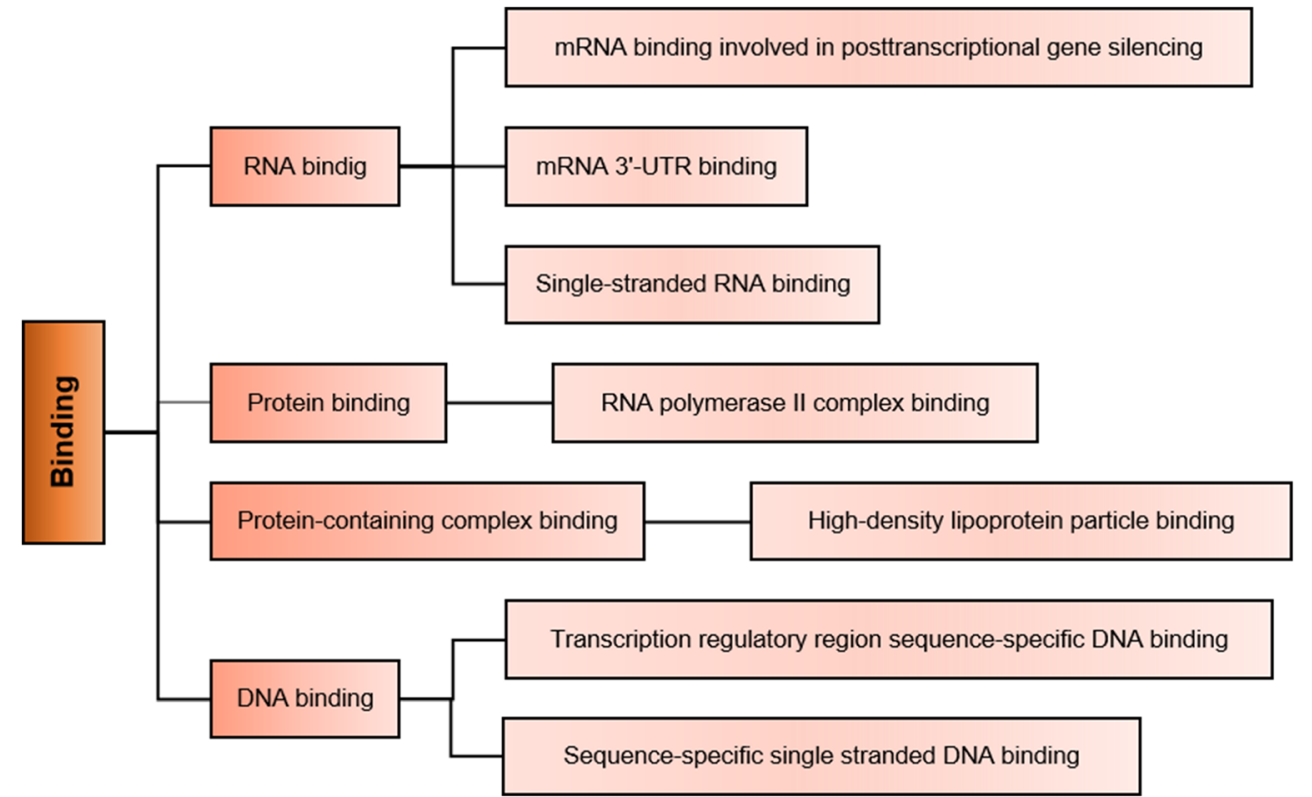



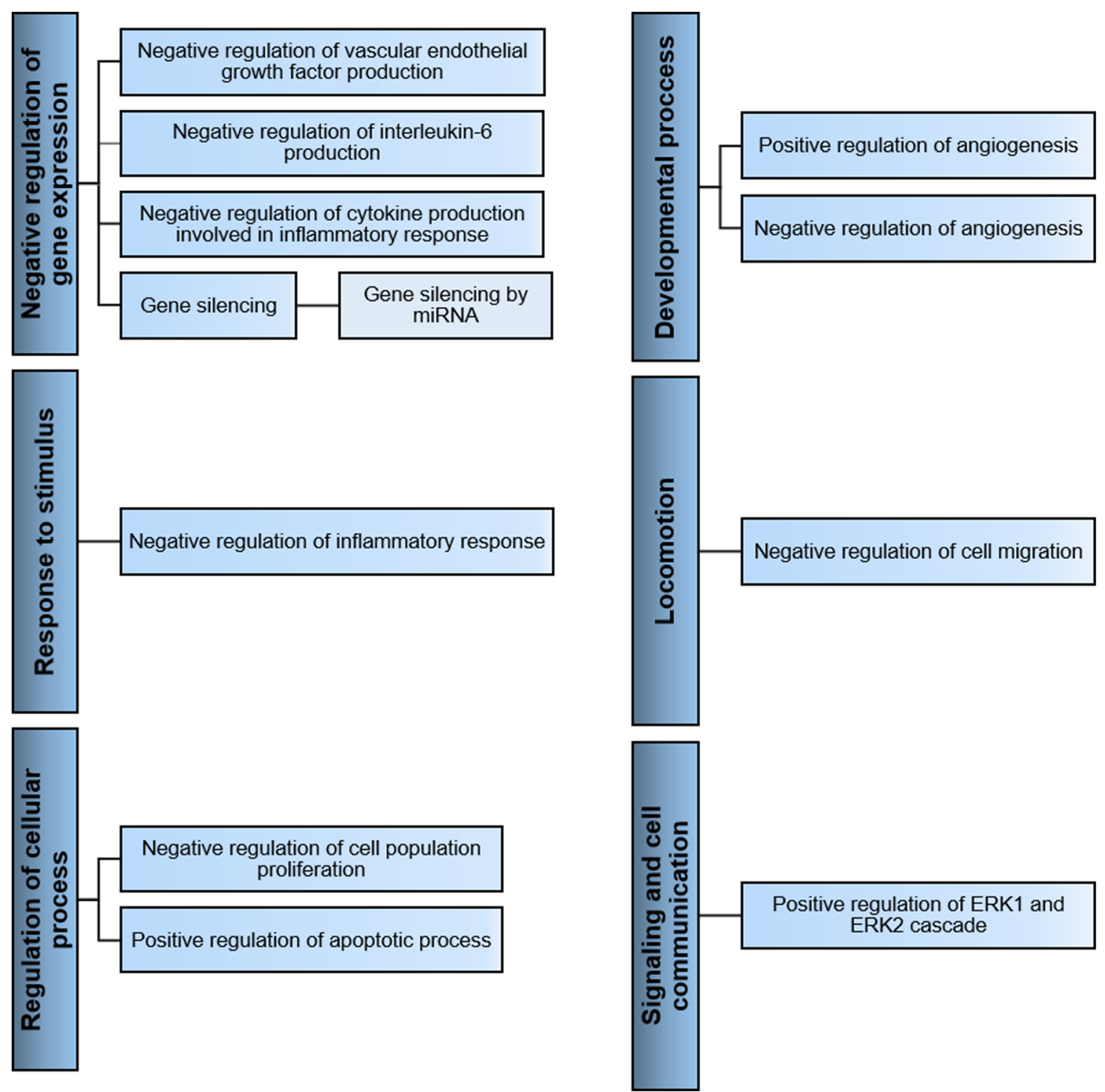

Fig. 7 Simplified outline of the main biological processes in which the miRNA detected in EVs derived from human AT-MSC are involved. For a complete review of the relationships between gene ontology terms see the chart view in the web-based tool QuickGO (https://www.ebi.ac.uk/QuickGO/)

Other types of small RNA, such as rRNA [54], snRNA, snoRNA [53, 54] and scRNA [53], are present in AT-MSCEVs, but the available information about these is even less than that of tRNA.

\section{Lipids}

The third type of molecule transported by EVs is lipids [3, 4]. The lipid composition of EVs has been less studied than that of proteins or miRNAs [8]. Thus, the number of lipid entries (639) in the Vesiclepedia database [41] is notably lower than the number of protein and miRNA entries $(349,988$ and 10,520 , respectively). None of these lipid entries are related to AT-MSC-EVs or any other MSC-EVs. The total lipid content of AT-MSC-EVs has been analysed by Bari et al. [58], using the Nile Red assay. However, to our knowledge, there is no detailed information about the different types of lipids present in AT-MSC-EVs.

\section{Modification of Cargo Components to Improve their Potential Effects}

Different cell culture conditions and pre-treatments have been used to modify the profile of human AT-MSC-EV cargo, with the aim to improve its effects in skin flap survival $[59,86]$, angiogenesis [60, 61, 64, 80], immune response [71, 87], bone regeneration [77] and cancer [118, 119]. To this purpose, human AT-MSCs have been exposed to oxidative stress [59, 86], hypoxic [61, 80] or inflammatory culture conditions [71, 87], stimulation with platelet-derived growth factor (PDGF) $[60,65]$ and basic fibroblast growth factor (bFGF) 


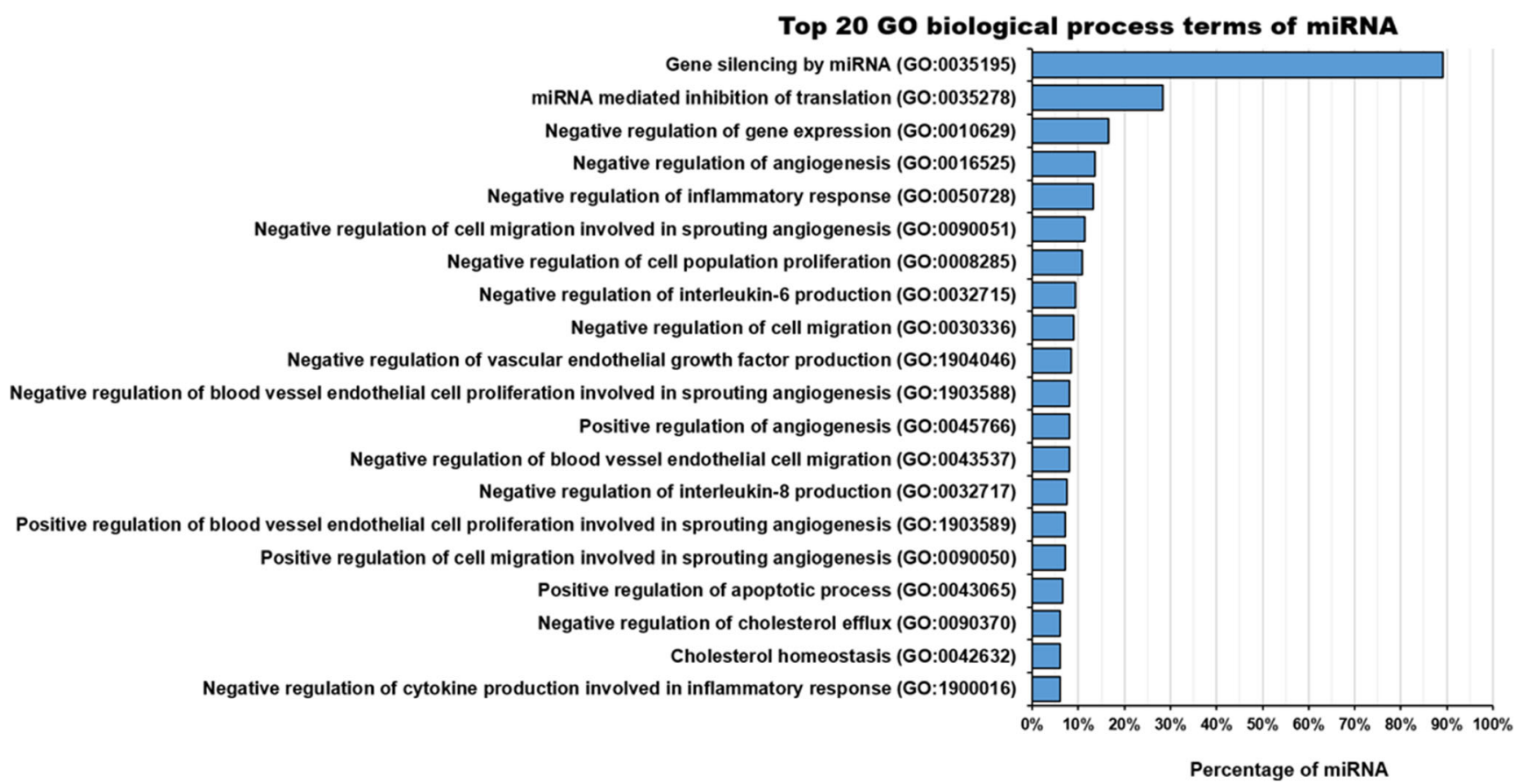

Fig. 8 The top 20 gene ontology (GO) biological process terms of the 212 miRNA detected in human AT-MSC-EVs which presented annotations in this aspect. The $89 \%$ of them are involved in gene silencing

[64] and transfected with lentiviral particles with different miRNAs [77, 118, 119].

Under oxidative stress conditions $\left(50 \mu \mathrm{M} \mathrm{H}_{2} \mathrm{O}_{2}\right)$, AT-MSC-EVs showed an enhanced effect on skin flap survival after ischemic injury in in vivo models [59, 86]. This improvement was associated with a promotion of angiogenesis, reduction of inflammation and apoptosis [86]. The proteomic analysis of these EVs showed an increase ( $>2$-fold) of histone H4, beta ig-h3, ITIHC2, FLG-2, periostin, thrombospondin-1, pentraxinrelated protein PTX3 and annexin A5; and a decrease (>2-fold) of plakophilin-1, VDB, Apo B-100, lactotransferrin, serotransferrin, alpha-fetoprotein, fatty acid-binding protein 5, dermcidin, and hornerin [59]. The RNA sequencing analysis showed that hsa-miR$10,395-5 p$ and hsa-miR-10,395-3p were increased in $\mathrm{H}_{2} \mathrm{O}_{2}$ AT-MSC-EVs, while hsa-miR-24-3p, hsa-miR16-5p, hsa-miR-93-5p, hsa-miR-31-5p, hsa-miR-23a-3p, hsa-miR-152-3p, hsa-miR-122-5p, hsa-miR-134-5p, hsamiR-221-3p, hsa-miR-196a-5p, hsa-miR-23b-3p, hsamiR-222-3p were decreased [59]. Finally, the peak size of EV from $\mathrm{H}_{2} \mathrm{O}_{2}$-stimulated AT-MSC was larger than that of unstimulated cells [59].

Hypoxic culture conditions also induce the release of larger EVs according to Han et al. [61], although other authors claim that there are no significant differences in size [80]. The EVs collected from AT-MSC cultured under hypoxic conditions $\left(5 \% \mathrm{O}_{2}\right)$ seemed to enhance angiogenic properties in cultured human umbilical vein endothelial cells and in an in vivo model of fat grafting $[61,80]$. The results of these studies showed that the amount of the surface marker CD44 was significantly lower in hypoxic EVs [80], while VEGF-A, EGF, FGF-4, VEGFR-2, VEGFR-3, C-C motif chemokine 8 and 13 were increased under these culture conditions [61].

EVs contents are also different after AT-MSC exposure to inflammatory cytokines. In EVs secreted by INF- $\gamma$-stimulated AT-MSC, indoleamine 2,3-dioxygenase mRNA was detected, although its presence did not significantly improve their potential to control activated $\mathrm{T}$ cell proliferation, in comparison with those derived from unstimulated AT-MSC [87]. However, when AT-MSCs were pretreated with both INF- $\gamma$ and TNF- $\alpha$, the enriched EVs induced the polarization of macrophages to the M2 phenotype [71]. Under this proinflammatory culture condition, AT-MSC-EVs cause differences in the expression of 81 different miRNAs [71] (Table 3).

Other methods used to alter the expression of cargo components are stimulation with PDGF [60, 65], with bFGF [64], and lentiviral transfection with the miRNA of interest [77, $118,119]$. In the former case, PDGF stimulation increased release of smaller AT-MSC-EVs, and improved their angiogenic potential, both in cultured human microvascular endothelial cells and in an in vivo model of severe combined immunodeficiency [60]. This stimulation also improved the ATMSC-EVs anti-inflammatory and immunomodulatory potential both in vitro and in vivo in peripheral blood mononuclear cell and in a murine model of hindlimb ischemia, respectively [65]. Regarding protein composition, these EVs contained several proteins not observed in unstimulated AT-MSCEVs: C-C motif chemokine 21, IL-17RD, IL-20RA, inhibin A, tyrosine-protein kinase Lck, LIF, SL-2, SL-3, MMP-14, 
Table 3 miRNA detected in EVs derived from human AT-MSC treated with IFN- $\gamma$ and TNF $\alpha$, PDGF and bFGF (Modified tables from Domenis et al., 2018 [71], Lopatina et al., 2014 and 2018, [64, 65])

Stimulation with IFN- $\gamma$ and TNF $\alpha$

miRNA under-expressed

$\begin{array}{lll}\begin{array}{l}\text { has-let-7b-5p } \\ \text { hsa-miR-10b-5p }\end{array} & \text { hsa-let-7c-5p } & \text { let-7f-5p } \\ \text { hsa-miR-16-5p } & \text { hsa-miR-125a-5p } & \text { hsa-miR-191-5p } \\ \text { hsa-miR-27a-3p } & \text { hsa-miR-28-3p } & \text { hsa-miR-21-5p } \\ \text { hsa-miR-92a-3p } & \text { hsa-miR-941 } & \text { hsa-miR-39b-5p } \\ \text { miRNA over-expressed } & & \\ \text { hsa-let-7a-5p } & \text { hsa-let-7 g-5p } & \text { hsa-miR-100-5p } \\ \text { hsa-miR-146a-5p } & \text { hsa-miR-148a-3p } & \text { hsa-miR-151a-3p } \\ \text { hsa-miR-199a-5p } & \text { hsa-miR-21-3p } & \text { hsa-miR-25-3p } \\ \text { hsa-miR-320a-3p } & \text { hsa-miR-340-5p } & \text { hsa-miR-378a-3p } \\ \text { hsa-miR-889-3p } & \text { hsa-miR-92b-3p } & \text { hsa-miR-99a-5p } \\ \text { Lost miRNA } & & \\ \text { hsa-let-7e-5p } & \text { hsa-miR-125b-5p } & \text { hsa-miR-134-5p } \\ \text { hsa-miR-150-5p } & \text { hsa-miR-151a-5p } & \text { hsa-miR-181b-5p } \\ \text { hsa-miR-193b-3p } & \text { hsa-miR-197-3p } & \text { hsa-miR-19b-3p } \\ \text { hsa-miR-27b-3p } & \text { hsa-miR-301a-3p } & \text { hsa-miR-30a-3p } \\ \text { hsa-miR-409-3p } & \text { hsa-miR-4677-3p } & \text { hsa-miR-532-5p } \\ \text { hsa-miR-671-3p } & \text { hsa-miR-7706 } & \text { hsa-miR-98-5p } \\ \text { Gained miRNA } & & \\ \text { hsa-miR-100-3p } & \text { hsa-miR-101-3p } & \text { hsa-miR-1246 } \\ \text { hsa-miR-155-5p } & \text { hsa-miR-361-5p } & \text { hsa-miR-411-5p }\end{array}$

has-let-7i-5p

hsa-miR-146b-5p

hsa-miR-22-3p

hsa-miR-423-5p

hsa-miR-125b-1-3p

hsa-miR-181a-5p

hsa-miR-26a-5p

hsa-miR-410-3p

hsa-miR-136-3p

hsa-miR-186-5p

hsa-miR-19b-3p

hsa-miR-335-3p

hsa-miR-6515-5p

hsa-miR-127-3p

hsa-miR-493-3p
hsa-miR-10a-5p

hsa-miR-148a-5p

hsa-miR-221-3p

hsa-miR-486-5p

hsa-miR-126a-5p

hsa-miR-192-5p

hsa-miR-30e-3p

hsa-miR-423-3p

hsa-miR-148b-3p

hsa-miR-1910-5p

hsa-miR-23b-3p

hsa-miR-382-5p

hsa-miR-654-5p

Stimulation with PDGF

miRNA under-expressed

hsa-miR-1225-3p

hsa-miR-1226-5p

miRNA over-expressed

hsa-miR-125b

hsa-miR-195

hsa-miR-203a-3p

hsa-miR-99a-3p

miRNA-expressed only in stimulated

hsa-let-7e

hsa-miR-129

hsa-miR-186

hsa-miR-221

hsa-miR-373

hsa-miR-511

hsa-miR-550

hsa-miR-579

hsa-miR-621

hsa-miR-872

Stimulation with bFGF

miRNA under-expressed

hsa-let-7a

hsa-miR-100

hsa-miR-10b

hsa-miR-138

hsa-miR-185

hsa-miR-199a

hsa-miR-210

\section{hsa-let-7f-2}

hsa-miR-1296

hsa-miR-202

hsa-miR-23b

hsa-miR-380-3p

hsa-miR-518a-5p

hsa-miR-551b

hsa-miR-581

hsa-miR-643

hsa-miR-92a-1
hsa-miR-122

hsa-miR-133b

hsa-miR-208b

hsa-miR-296-3p

hsa-miR-381

hsa-miR-525-3p

hsa-miR-562

hsa-miR-582-5p

hsa-miR-708-3p

hsa-miR-943 hsa-let-7b

hsa-miR-101

hsa-miR-125a-3p

hsa-miR-143

hsa-miR-192

hsa-miR-199a-3p

hsa-miR-22 hsa-let-7d

hsa-miR-103

hsa-miR-125b

hsa-miR-15b

hsa-miR-193a-5p

hsa-miR-20a

hsa-miR-221
hsa-miR-1269

hsa-miR-147b

hsa-miR-211

hsa-miR-338-5P

hsa-miR-432

hsa-miR-548c-5p

hsa-miR-567

hsa-miR-604

hsa-miR-765

hsa-miR-944
hsa-miR-1276

hsa-miR-154

hsa-miR-216b

hsa-miR-34b

hsa-miR-502

hsa-miR-548I

hsa-miR-575

hsa-miR-614

hsa-miR-802 hsa-let-7e

hsa-miR-106a

hsa-miR-127

hsa-miR-17

hsa-miR-194

hsa-miR-20b

hsa-miR-224 hsa-let-7 g

hsa-miR-10a

hsa-miR-130a

hsa-miR-181a

hsa-miR-195

hsa-miR-21

hsa-miR-26a 
Table 3 (continued)

hsa-miR-27b

miRNA-expressed only in stimulated

hsa-let-7c hsa-miR-130b

hsa-miR-199b hsa-miR-223

hsa-miR-340 hsa-miR-381

hsa-miR-579

hsa-miR-133a

hsa-miR-26b

hsa-miR-483-5p

hsa-miR-654-3p
hsa-miR-184

hsa-miR-27a

hsa-miR-539

hsa-miR-885-5p
hsa-miR-193a-3p

hsa-miR-28-3p

hsa-miR-542-5p
OSM, kit ligand, IL-6RB (soluble form), TGF-beta 5 (not found in UniProtKB), thrombopoietin, metalloproteinase inhibitor 1, and TNF receptor superfamily member 10D [60]. In addition, 65 proteins were up-regulated and 15 proteins were down-regulated (Table 4). The miRNA composition of stimulated AT-MSC-EVs also showed variations in the expression of 55 different miRNAs [65] (Table 3).

Table 4 Protein detected in EVs derived from human AT-MSC treated with PDGF (Modified table from Lopatina et al., 2018, [65])

Stimulation with PDGF

Proteins up-regulated

Adenomatous polyposis coli protein*

Calsyntenin-1

C-C motif chemokine $1 *$

Coagulation factor XIII B chain

C-X-C motif chemokine 11*

Insulin-degrading enzyme*

Interleukin-23 subunit alpha*

Lymphotoxin beta

Matrix metalloproteinase-9*

Neurogenic differentiation factor $1 *$

Platelet-derived growth factor D*

Secreted frizzled-related protein $1 *$

Toll-like receptor $2 *$

Transforming growth factor beta-1

Tumor necrosis factor ligand superfamily member $15^{*}$

Vascular endothelial growth factor $\mathrm{A}^{*}$

Vascular endothelial growth factor receptor $2 *$

Proteins down-regulated

Activin receptor type-1*

SWI/SNF-related matrix-associated actin-dependent regulator of chromatin subfamily E member 1 *

Calbindin

Receptor-interacting serine/threonine-protein kinase $1^{*}$
ADP-ribosyl cyclase/cyclic

ADP-ribose hydrolase $1^{*}$

Calsyntenin-1

C-C motif chemokine 22*

Complement C3*

Cystatin A

Interleukin-13 receptor subunit alpha-2*

Keratin, type I cytoskeletal 19* Kremen protein 2*

Macrophage migration inhibitory Matrilysin* factor*

Metalloproteinase inhibitor 3*

Neurturin

Polyubiquitin-B*

Sialic acid-binding Ig-like lectin $5^{*}$

Toll-like receptor $4 *$

Triggering receptor expressed on myeloid cells $1 *$

Tumor necrosis factor ligand superfamily member $8^{*}$

Vascular endothelial growth factor $\mathrm{A}^{*}$

\section{MHC class I polypeptide-related} sequence $\mathrm{A}^{*}$

Neutrophil-activating peptide 2

Progranulin

Stromelysin-2*

Transferrin receptor protein $1 *$

Tumor necrosis factor ligand superfamily member 10 *

Tumor necrosis factor receptor superfamily member $19 *$

Vascular endothelial growth factor $\mathrm{C}^{*}$

Angiopoietin-related protein $2^{*}$

Beta-2-microglobulin*

umor necrosis factor receptor superfamily member $13 C^{*}$

Fibroblast growth factor receptor $3 *$

Transcription initiation factor TFIID subunit 4* protein*

$\mathrm{T}$ lymphocyte activation antigen CD80*
Cadherin-1*

C-C chemokine receptor type $7^{*}$

Ceruloplasmin

C-X-C motif chemokine $10 *$

HLA class II histocompatibility antigen gamma chain*

Interleukin-21 receptor*

Low-density lipoprotein receptor*

Matrix metalloproteinase-14*

Neural cell adhesion molecule $1 *$

Orexin receptor type $1 *$

Protein S100-A10

Thrombopoietin

Transforming growth factor beta receptor type $3 *$

Tumor necrosis factor ligand superfamily member 11 *

Tumor necrosis factor receptor superfamily member 27 *

Vascular endothelial growth factor D*

Apolipoprotein C-II*

Bone morphogenetic protein $7 *$

pro-Glucagon

*The referred article used alternative or short names 
The stimulation with bFGF did not affect the number or size of released AT-MSC-EVs but it reduced their antigenic properties, stimulating the stabilization of vessel growth, both in cultured human microvascular endothelial cells and in an in vivo model of severe combined immunodeficiency [64]. The analysis of these EVs showed that angiogenic and antiangiogenic proteins such as tumor necrosis factor ligand superfamily member 13, artemin, lactadherin, MMP-20, angiopoietin-related protein 7 , thrombospondin, angiostatin and endostatin were lost, while new angiogenesis modulatory proteins, such as tumor necrosis factor ligand superfamily member 11 and matrilysin were gained. Regarding miRNA profile, differences in the expression of 55 different miRNAs were observed [64] (Table 3).

Finally, AT-MSC-EVs have been transfected with lentiviral particles to produce EVs enriched in miRNA 375 [77], miRNA-125b [119] and miRNA 101 [118]. The miRNA-375-enriched EVs promoted bone regeneration in an in vivo model of calvarial defects. AT-MSC-EVs enriched in miRNA-125b [119] and miRNA 101 [118] induced a reduction in cell proliferation of hepatocellular carcinoma cells and inhibited osteosarcoma cell invasion and migration in vitro, respectively. In addition, miRNA-101-enriched EVs also induced inhibition of osteosarcoma metastasis in a lung metastasis model in vivo [118].

\section{Conclusions}

There is an increasing interest in the study of EVs as new therapeutic options in several research fields, due to their role in different biological processes, including cell proliferation, apoptosis, angiogenesis, inflammation and immune response, among others. Their potential is based upon the molecules transported inside these particles. Therefore, both molecule identification and an understanding of the molecular functions and biological processes in which they are involved are essential to advance this area of research. To the best of our knowledge, the presence of 591 proteins and 604 miRNAs in human AT-MSC-EVs has been described. The most important molecular function enabled by them is the binding function, which supports their role in cell communication. Regarding the biological processes, the proteins detected are mainly involved in signal transduction, while most miRNAs take part in negative regulation of gene expression. The involvement of both molecules in essential biological processes such as inflammation, angiogenesis, cell proliferation, apoptosis and migration, supports the beneficial effects of human ATMSC-EVs observed in both in vitro and in vivo studies, in diseases of the musculoskeletal and cardiovascular systems, kidney, and skin.

Interestingly, the contents of AT-MSC-EVs can be modified by cell stimulation and different cell culture conditions, such as oxidative stress or hypoxia, to engineer a cargo selection with improved antigenic, anti-inflammatory or immunosuppressive effects. Moreover, it is also possible to enrich specific miRNAs in the cargo via transfection of AT-MSC with lentiviral particles. These modifications have enhanced the positive effects in skin flap survival, immune response, bone regeneration and cancer treatment. This phenomenon opens new avenues to examine the therapeutic potential of AT-MSC-EVs.

Abbreviations Apo B-100, apolipoprotein B-100; AT, adipose tissue; AT-MSC-EVs, adipose mesenchymal cell-derived extracellular vesicles; Beta ig-h3, transforming growth factor-beta-induced protein ig-h3; bFGF, basic fibroblast growth factor; BMP-1, bone morphogenetic protein 1; BMPR-1A, bone morphogenetic protein receptor type-1A; BMPR-2, bone morphogenetic protein receptor type-2; BM, bone marrow; BM-MSC, bone marrow mesenchymal stem cells; EF-1-alpha-1, elongation factor 1-alpha 1; EF-2, elongation factor 2; EGF, epidermal growth factor; EMBL-EBI, the European Bioinformatics Institute; EV, extracellular vesicle; FGF-4, fibroblast growth factor 4; FGFR-1, fibroblast growth factor receptor 1; FGFR-4, fibroblast growth factor receptor 4; FLG-2, filaggrin-2; G alpha-13, guanine nucleotide-binding protein subunit alpha-13; GAPDH, glyceraldehyde 3-phosphate dehydrogenase; GO, gene ontology; IBP-7, insulin-like growth factor-binding protein 7; IL-1 alpha, interleukin-1 alpha; IL-4, interleukin-4; IL-6, interleukin-6; IL-6RB, interleukin-6 receptor subunit beta; IL-10, interleukin-10; IL17RD, interleukin-17 receptor D; IL-20RA, interleukin-20 receptor subunit alpha; ISEV, International Society for Extracellular Vesicles; ITI$\mathrm{HC} 2$, inter-alpha-trypsin inhibitor heavy chain $\mathrm{H} 2$; LIF, leukemia inhibitory factor; LTBP-1, latent-transforming growth factor beta-binding protein 1; MAP kinase 1, mitogen-activated protein kinase 1; MAP kinase 3, mitogen-activated protein kinase 3; miRNA, microRNA; MMP-9, matrix metalloproteinase-9; MMP-14, matrix metalloproteinase-14; MMP-20, matrix metalloproteinase-20; mRNA, messenger RNA; MSC, mesenchymal stem cells; OSM, oncostatin-M; PDGF, platelet-derived growth factor; PDGFR-alpha, platelet-derived growth factor receptor alpha.; PDGFR-beta, platelet-derived growth factor receptor beta; rRNA, small ribosomal RNA; SCFR, mast/stem cell growth factor receptor Kit; scRNA, small cytoplasmic RNA; SL-2, stromelysin-2; SL-3, stromelysin-3; snRNA, small nuclear RNA; snoRNA, small nucleolar RNA; TGFR-2, TGF-beta receptor type-2; tRNA, transfer RNA; UniProtKB, Universal Protein Knowledgebase; VDB, vitamin Dbinding protein; VEGF-A, vascular endothelial growth factor A; VEGFR-2, vascular endothelial growth factor receptor 2; VEGFR-3, vascular endothelial growth factor receptor

Supplementary Information The online version contains supplementary material available at https://doi.org/10.1007/s12015-021-10155-5.

Acknowledgements The authors acknowledge Dr. Teresa Nieto-Miguel and Dr. Sara Galindo for critical reading of the manuscript. English grammar and spelling of this manuscript have been professionally revised and corrected by Proof-Reading-Service (Hertfordshire, United Kingdom).

Availability of Data and Materials The data used to support the findings of this review are available from the corresponding author upon request.

Author's Contributions All authors contributed to the study conception and design. Literature search and data analysis were performed by MLAA. The first draft of the manuscript was written by MLAA and MLAA, LGP and YD commented on previous versions of the manuscript. MLAA, LGP and YD read and approved the final manuscript. 
Funding This work was supported by Ministerio de Ciencia, Innovación y Universidades (MCIU), Agencia Estatal de Investigación (AEI) and Fondo Europeo de Desarrollo Regional (FEDER), Grant number RTI2018-094071-B-C21.

\section{Declarations}

Ethical Approval Not applicable.

Consent to Participate Not applicable.

Consent to Publish Not applicable.

Competing Interests The authors declare that they have no conflict of interest.

Open Access This article is licensed under a Creative Commons Attribution 4.0 International License, which permits use, sharing, adaptation, distribution and reproduction in any medium or format, as long as you give appropriate credit to the original author(s) and the source, provide a link to the Creative Commons licence, and indicate if changes were made. The images or other third party material in this article are included in the article's Creative Commons licence, unless indicated otherwise in a credit line to the material. If material is not included in the article's Creative Commons licence and your intended use is not permitted by statutory regulation or exceeds the permitted use, you will need to obtain permission directly from the copyright holder. To view a copy of this licence, visit http://creativecommons.org/licenses/by/4.0/.

\section{References}

1. Théry, C., Witwer, K. W., Aikawa, E., Alcaraz, M. J., Anderson, J. D., Andriantsitohaina, R., Antoniou, A., Arab, T., Archer, F., Atkin-Smith, G. K., Ayre, D. C., Bach, J. M., Bachurski, D., Baharvand, H., Balaj, L., Baldacchino, S., Bauer, N. N., Baxter, A. A., Bebawy, M., Beckham, C., Bedina Zavec, A., Benmoussa, A., Berardi, A. C., Bergese, P., Bielska, E., Blenkiron, C., BobisWozowicz, S., Boilard, E., Boireau, W., Bongiovanni, A., Borràs, F. E., Bosch, S., Boulanger, C. M., Breakefield, X., Breglio, A. M., Brennan, M. Á., Brigstock, D. R., Brisson, A., Broekman, M. L. D., Bromberg, J. F., Bryl-Górecka, P., Buch, S., Buck, A. H., Burger, D., Busatto, S., Buschmann, D., Bussolati, B., Buzás, E. I., Byrd, J. B., Camussi, G., Carter, D. R. F., Caruso, S., Chamley, L. W., Chang, Y. T., Chen, C., Chen, S., Cheng, L., Chin, A. R., Clayton, A., Clerici, S. P., Cocks, A., Cocucci, E., Coffey, R. J., Cordeiro-da-Silva, A., Couch, Y., Coumans, F. A. W., Coyle, B., Crescitelli, R., Criado, M. F., D'Souza-Schorey, C., Das, S., Datta Chaudhuri, A., de Candia, P., de Santana Jr., E. F., de Wever, O., del Portillo, H. A., Demaret, T., Deville, S., Devitt, A., Dhondt, B., di Vizio, D., Dieterich, L. C., Dolo, V., Dominguez Rubio, A. P., Dominici, M., Dourado, M. R., Driedonks, T. A. P., Duarte, F. V., Duncan, H. M., Eichenberger, R. M., Ekström, K., el Andaloussi, S., Elie-Caille, C., Erdbrügger, U., Falcón-Pérez, J. M., Fatima, F., Fish, J. E., Flores-Bellver, M., Försönits, A., Frelet-Barrand, A., Fricke, F., Fuhrmann, G., Gabrielsson, S., Gámez-Valero, A., Gardiner, C., Gärtner, K., Gaudin, R., Gho, Y. S., Giebel, B., Gilbert, C., Gimona, M., Giusti, I., Goberdhan, D. C. I., Görgens, A., Gorski, S. M., Greening, D. W., Gross, J. C., Gualerzi, A., Gupta, G. N., Gustafson, D., Handberg, A., Haraszti, R. A., Harrison, P., Hegyesi, H., Hendrix, A., Hill, A. F., Hochberg, F. H., Hoffmann, K. F., Holder, B., Holthofer, H.,
Hosseinkhani, B., Hu, G., Huang, Y., Huber, V., Hunt, S., Ibrahim, A. G. E., Ikezu, T., Inal, J. M., Isin, M., Ivanova, A., Jackson, H. K., Jacobsen, S., Jay, S. M., Jayachandran, M., Jenster, G., Jiang, L., Johnson, S. M., Jones, J. C., Jong, A., Jovanovic-Talisman, T., Jung, S., Kalluri, R., Kano, S. I., Kaur, S., Kawamura, Y., Keller, E. T., Khamari, D., Khomyakova, E., Khvorova, A., Kierulf, P., Kim, K. P., Kislinger, T., Klingeborn, M., Klinke II, D. J., Kornek, M., Kosanović, M. M., Kovács, Á. F., Krämer-Albers, E. M., Krasemann, S., Krause, M., Kurochkin, I. V., Kusuma, G. D., Kuypers, S., Laitinen, S., Langevin, S. M., Languino, L. R., Lannigan, J., Lässer, C., Laurent, L. C., Lavieu, G., Lázaro-Ibáñez, E., le Lay, S., Lee, M. S., Lee, Y. X. F., Lemos, D. S., Lenassi, M., Leszczynska, A., Li, I. T. S., Liao, K., Libregts, S. F., Ligeti, E., Lim, R., Lim, S. K., Linē, A., Linnemannstöns, K., Llorente, A., Lombard, C. A., Lorenowicz, M. J., Lörincz, Á. M., Lötvall, J., Lovett, J., Lowry, M. C., Loyer, X., Lu, Q., Lukomska, B., Lunavat, T. R., Maas, S. L. N., Malhi, H., Marcilla, A., Mariani, J., Mariscal, J., Martens-Uzunova, E. S., Martin-Jaular, L., Martinez, M. C., Martins, V. R., Mathieu, M., Mathivanan, S., Maugeri, M., McGinnis, L. K., McVey, M. J., Meckes Jr., D. G., Meehan, K. L., Mertens, I., Minciacchi, V. R., Möller, A., Møller Jørgensen, M., Morales-Kastresana, A., Morhayim, J., Mullier, F., Muraca, M., Musante, L., Mussack, V., Muth, D. C., Myburgh, K. H., Najrana, T., Nawaz, M., Nazarenko, I., Nejsum, P., Neri, C., Neri, T., Nieuwland, R., Nimrichter, L., Nolan, J. P., Nolte-'t Hoen, E. N. M., Noren Hooten, N., O'Driscoll, L., O'Grady, T., O'Loghlen, A., Ochiya, T., Olivier, M., Ortiz, A., Ortiz, L. A., Osteikoetxea, X., Østergaard, O., Ostrowski, M., Park, J., Pegtel, D. M., Peinado, H., Perut, F., Pfaffl, M. W., Phinney, D. G., Pieters, B. C. H., Pink, R. C., Pisetsky, D. S., Pogge von Strandmann, E., Polakovicova, I., Poon, I. K. H., Powell, B. H., Prada, I., Pulliam, L., Quesenberry, P., Radeghieri, A., Raffai, R. L., Raimondo, S., Rak, J., Ramirez, M. I., Raposo, G., Rayyan, M. S., RegevRudzki, N., Ricklefs, F. L., Robbins, P. D., Roberts, D. D., Rodrigues, S. C., Rohde, E., Rome, S., Rouschop, K. M. A., Rughetti, A., Russell, A. E., Saá, P., Sahoo, S., Salas-Huenuleo, E., Sánchez, C., Saugstad, J. A., Saul, M. J., Schiffelers, R. M., Schneider, R., Schøyen, T. H., Scott, A., Shahaj, E., Sharma, S., Shatnyeva, O., Shekari, F., Shelke, G. V., Shetty, A. K., Shiba, K., Siljander, P. R. M., Silva, A. M., Skowronek, A., Snyder II, O. L., Soares, R. P., Sódar, B. W., Soekmadji, C., Sotillo, J., Stahl, P. D., Stoorvogel, W., Stott, S. L., Strasser, E. F., Swift, S., Tahara, H., Tewari, M., Timms, K., Tiwari, S., Tixeira, R., Tkach, M., Toh, W. S., Tomasini, R., Torrecilhas, A. C., Tosar, J. P., Toxavidis, V., Urbanelli, L., Vader, P., van Balkom, B. W. M., van der Grein, S. G., van Deun, J., van Herwijnen, M. J. C., van Keuren-Jensen, K., van Niel, G., van Royen, M. E., van Wijnen, A. J., Vasconcelos, M. H., Vechetti Jr., I. J., Veit, T. D., Vella, L. J., Velot, É., Verweij, F. J., Vestad, B., Viñas, J. L., Visnovitz, T., Vukman, K. V., Wahlgren, J., Watson, D. C., Wauben, M. H. M., Weaver, A., Webber, J. P., Weber, V., Wehman, A. M., Weiss, D. J., Welsh, J. A., Wendt, S., Wheelock, A. M., Wiener, Z., Witte, L., Wolfram, J., Xagorari, A., Xander, P., Xu, J., Yan, X., Yáñez-Mó, M., Yin, H., Yuana, Y., Zappulli, V., Zarubova, J., Žèkas, V., Zhang, J. Y., Zhao, Z., Zheng, L., Zheutlin, A. R., Zickler, A. M., Zimmermann, P., Zivkovic, A. M., Zocco, D., \& Zuba-Surma, E. K. (2018). Minimal information for studies of extracellular vesicles 2018 (MISEV2018): A position statement of the International Society for Extracellular Vesicles and update of the MISEV2014 guidelines. Journal of Extracellular Vesicles, 7(1), 1535750. https://doi.org/10.1080/20013078.2018.1535750.

2. Witwer, K. W., \& Théry, C. (2019). Extracellular vesicles or exosomes? On primacy, precision, and popularity influencing a choice of nomenclature. Journal of 
Extracellular Vesicles, 8(1), 1648167. https://doi.org/10. 1080/20013078.2019.1648167.

3. Kalra, H., Drummen, G., \& Mathivanan, S. (2016). Focus on extracellular vesicles: Introducing the next small big thing. International Journal of Molecular Sciences, 17(2), 170. https:// doi.org/10.3390/ijms17020170.

4. Edgar, J. R. (2016). Q\&A: What are exosomes, exactly? BMC Biology, 14(1), 46. https://doi.org/10.1186/s12915-016-0268-z.

5. Aryani, A., \& Denecke, B. (2016). Exosomes as a Nanodelivery system: A key to the future of Neuromedicine? Molecular Neurobiology, 53(2), 818-834. https://doi.org/10.1007/s12035014-9054-5.

6. Mathieu, M., Martin-Jaular, L., Lavieu, G., \& Théry, C. (2019). Specificities of secretion and uptake of exosomes and other extracellular vesicles for cell-to-cell communication. Nature Cell Biology, 21(1), 9-17. https://doi.org/10.1038/s41556-018-0250-9.

7. Lötvall, J., Hill, A. F., Hochberg, F., Buzás, E. I., Di Vizio, D., Gardiner, C., et al. (2014). Minimal experimental requirements for definition of extracellular vesicles and their functions: A position statement from the International Society for Extracellular Vesicles. Journal of Extracellular Vesicles, 3(1), 26913. https:// doi.org/10.3402/jev.v3.26913.

8. Colombo, M., Raposo, G., \& Théry, C. (2014). Biogenesis, secretion, and intercellular interactions of Exosomes and other extracellular vesicles. Annual Review of Cell and Developmental Biology, 30(1), 255-289. https://doi.org/10.1146/annurevcellbio-101512-122326.

9. Hong, P., Yang, H., Wu, Y., Li, K., \& Tang, Z. (2019). The functions and clinical application potential of exosomes derived from adipose mesenchymal stem cells: A comprehensive review. Stem Cell Research \& Therapy, 10(1), 242. https://doi.org/10. 1186/s13287-019-1358-y.

10. Yeo, R. W. Y., Lai, R. C., Zhang, B., Tan, S. S., Yin, Y., Teh, B. J., \& Lim, S. K. (2013). Mesenchymal stem cell: An efficient mass producer of exosomes for drug delivery. Advanced Drug Delivery Reviews, 65(3), 336-341. https://doi.org/10.1016/j.addr.2012.07. 001

11. Baglio, S. R., Rooijers, K., Koppers-Lalic, D., Verweij, F. J., Lanzón, M. P., Zini, N., et al. (2015). Human bone marrow-and adiposemesenchymal stem cells secrete exosomes enriched in distinctive miRNA and tRNA species. Stem Cell Research and Therapy, 6(127), 1-20. https://doi.org/10.1186/s13287-0150116-z.

12. Mitchell, R., Mellows, B., Sheard, J., Antonioli, M., Kretz, O., Chambers, D., Zeuner, M. T., Tomkins, J. E., Denecke, B., Musante, L., Joch, B., Debacq-Chainiaux, F., Holthofer, H., Ray, S., Huber, T. B., Dengjel, J., de Coppi, P., Widera, D., \& Patel, K. (2019). Secretome of adipose-derived mesenchymal stem cells promotes skeletal muscle regeneration through synergistic action of extracellular vesicle cargo and soluble proteins. Stem Cell Research \& Therapy, 10(1), 116. https://doi.org/10. 1186/s13287-019-1213-1.

13. Toh, W. S., Lai, R. C., Zhang, B., \& Lim, S. K. (2018). MSC exosome works through a protein-based mechanism of action. Biochemical Society Transactions, 46(4), 843-853. https://doi. org/10.1042/BST20180079.

14. Rastegar, F., Shenaq, D., Huang, J., Zhang, W., Zhang, B., He, B., Chen, L., Zuo, G. W., Luo, Q., Shi, Q., Wagner, E. R., Huang, E., Gao, Y., Gao, J. L., Kim, S. H., Zhou, J. Z., Bi, Y., Su, Y., Zhu, G., Luo, J., Luo, X., Qin, J., Reid, R. R., Luu, H. H., Haydon, R. C., Deng, Z. L., \& He, T. C. (2010). Mesenchymal stem cells: Molecular characteristics and clinical applications. World Journal of Stem Cells, 2(4), 67-80. https://doi.org/10.4252/wjsc. v2.i4.67.

15. Ng, T. K., Fortino, V. R., Pelaez, D., \& Cheung, H. S. (2014). Progress of mesenchymal stem cell therapy for neural and retinal diseases. World Journal of Stem Cells, 6(2), 111-119. https://doi. org/10.4252/wjsc.v6.i2.111.

16. Kern, S., Eichler, H., Stoeve, J., Klüter, H., \& Bieback, K. (2006). Comparative analysis of Mesenchymal stem cells from bone marrow, umbilical cord blood, or adipose tissue. Stem Cells, 24(5), 1294-1301. https://doi.org/10.1634/stemcells.2005-0342.

17. Kim, D. S., Lee, M. W., Yoo, K. H., Lee, T.-H., Kim, H. J., Jang, I. K., Chun, Y. H., Kim, H. J., Park, S. J., Lee, S. H., Son, M. H., Jung, H. L., Sung, K. W., \& Koo, H. H. (2014). Gene expression profiles of human adipose tissue-derived Mesenchymal stem cells are modified by cell culture density. PLoS One, 9(1), e83363. https://doi.org/10.1371/journal.pone.0083363.

18. Li, C., Wu, X., Tong, J., Yang, X., Zhao, J., Zheng, Q., Zhao, G. B., \& Ma, Z. J. (2015). Comparative analysis of human mesenchymal stem cells from bone marrow and adipose tissue under xeno-free conditions for cell therapy. Stem Cell Research \& Therapy, 6(1), 55. https://doi.org/10.1186/s13287-015-0066-5.

19. Mushahary, D., Spittler, A., Kasper, C., Weber, V., \& Charwat, V. (2018). Isolation, cultivation, and characterization of human mesenchymal stem cells. Cytometry Part A, 93(1), 19-31. https://doi. org/10.1002/cyto.a.23242.

20. Friedenstein, A. J., Petrakova, K. V., Kurolesova, A. I., \& Frolova, G. P. (1968). Heterotopic of bone marrow. Analysis of precursor cells for osteogenic and hematopoietic tissues. Transplantation, 6(2), 230-247 Retrieved from http://www.ncbi.nlm.nih.gov/ pubmed/5654088.

21. Strioga, M., Viswanathan, S., Darinskas, A., Slaby, O., \& Michalek, J. (2012). Same or not the same? Comparison of adipose tissue-derived versus bone marrow-derived Mesenchymal stem and stromal cells. Stem Cells and Development, 21(14), 2724-2752. https://doi.org/10.1089/scd.2011.0722.

22. Bunnell, B. A., Flaat, M., Gagliardi, C., Patel, B., \& Ripoll, C. (2008). Adipose-derived stem cells: Isolation, expansion and differentiation. Methods, 45(2), 115-120. https://doi.org/10.1016/j. ymeth.2008.03.006.

23. Bhattacharya, S., Gangaraju, R., \& Chaum, E. (2017). Recent advances in retinal stem cell therapy. Current Molecular Biology Reports, 3(3), 172-182. https://doi.org/10.1007/s40610-0170069-3.

24. Liu, L., Zhang, H., Mao, H., Li, X., \& Hu, Y. (2019). Exosomal miR-320d derived from adipose tissue-derived MSCs inhibits apoptosis in cardiomyocytes with atrial fibrillation (AF). Artificial Cells, Nanomedicine, and Biotechnology, 47(1), 3976-3984. https://doi.org/10.1080/21691401.2019.1671432.

25. Bonafede, R., Brandi, J., Manfredi, M., Scambi, I., Schiaffino, L., Merigo, F., Turano, E., Bonetti, B., Marengo, E., Cecconi, D., \& Mariotti, R. (2019). The anti-apoptotic effect of ASC-Exosomes in an in vitro ALS model and their proteomic analysis. Cells, 8(9), 1087. https://doi.org/10.3390/cells8091087.

26. Qu, Y., Zhang, Q., Cai, X., Li, F., Ma, Z., Xu, M., \& Lu, L. (2017). Exosomes derived from miR-181-5p-modified adiposederived mesenchymal stem cells prevent liver fibrosis via autophagy activation. Journal of Cellular and Molecular Medicine, 21(10), 2491-2502. https://doi.org/10.1111/jcmm.13170.

27. Shen, H., Yoneda, S., Abu-Amer, Y., Guilak, F., \& Gelberman, R. H. (2020). Stem cell-derived extracellular vesicles attenuate the early inflammatory response after tendon injury and repair. Journal of Orthopaedic Research, 38(1), 117-127. https://doi. org/10.1002/jor.24406.

28. Xing, X., Han, S., Cheng, G., Ni, Y., Li, Z., \& Li, Z. (2020). Proteomic analysis of Exosomes from adipose-derived Mesenchymal stem cells: A novel therapeutic strategy for tissue injury. BioMed Research International, 2020, 6094562-6094510. https://doi.org/10.1155/2020/6094562.

29. Yu, B., Shao, H., Su, C., Jiang, Y., Chen, X., Bai, L., Zhang, Y., Li, Q., Zhang, X., \& Li, X. (2016). Exosomes derived from MSCs 
ameliorate retinal laser injury partially by inhibition of MCP-1. Scientific Reports, 6(1), 34562. https://doi.org/10.1038/ srep34562.

30. Kim, S.-D., Kang, S. A., Kim, Y.-W., Yu, H. S., Cho, K.-S., \& Roh, H.-J. (2020). Screening and functional pathway analysis of pulmonary genes associated with suppression of allergic airway inflammation by adipose stem cell-derived extracellular vesicles. Stem Cells International, 2020, 1-11. https://doi.org/10.1155/ 2020/5684250.

31. Li, T., Zhou, X., Wang, J., Liu, Z., Han, S., Wan, L., Sun, X., \& Chen, H. (2020). Adipose-derived mesenchymal stem cells and extracellular vesicles confer antitumor activity in preclinical treatment of breast cancer. Pharmacological Research, 157, 104843. https://doi.org/10.1016/j.phrs.2020.104843.

32. Duan, Y., Luo, Q., Wang, Y., Ma, Y., Chen, F., Zhu, X., \& Shi, J. (2020). Adipose mesenchymal stem cell-derived extracellular vesicles containing microRNA-26a-5p target TLR4 and protect against diabetic nephropathy. Journal of Biological Chemistry, 1-32. https://doi.org/10.1074/jbc.RA120.012522.

33. Chang, C.-L., Chen, C.-H., Chiang, J. Y., Sun, C.-K., Chen, Y.-L., Chen, K.-H., et al. (2019). Synergistic effect of combined melatonin and adipose-derived mesenchymal stem cell (ADMSC)-derived exosomes on amelioration of dextran sulfate sodium (DSS)-induced acute colitis. American journal of translational research, 11(5), 2706-2724 Retrieved from http://www.ncbi. nlm.nih.gov/pubmed/31217848.

34. Deng, S., Zhou, X., Ge, Z., Song, Y., Wang, H., Liu, X., \& Zhang, D. (2019). Exosomes from adipose-derived mesenchymal stem cells ameliorate cardiac damage after myocardial infarction by activating S1P/SK1/S1PR1 signaling and promoting macrophage M2 polarization. The International Journal of Biochemistry \& Cell Biology, 114, 105564. https://doi.org/10.1016/j.biocel.2019. 105564.

35. Eirin, A., Riester, S. M., Zhu, X.-Y., Tang, H., Evans, J. M., O'Brien, D., et al. (2014). MicroRNA and mRNA cargo of extracellular vesicles from porcine adipose tissue-derived mesenchymal stem cells. Gene, 551(1), 55-64. https://doi.org/10.1016/j. gene.2014.08.041

36. Eirin, A., Zhu, X.-Y., Puranik, A. S., Woollard, J. R., Tang, H., Dasari, S., Lerman, A., van Wijnen, A. J., \& Lerman, L. O. (2016). Comparative proteomic analysis of extracellular vesicles isolated from porcine adipose tissue-derived mesenchymal stem/stromal cells. Scientific Reports, 6(1), 36120. https://doi.org/10.1038/ srep36120.

37. Pawar, A. S., Eirin, A., Tang, H., Zhu, X.-Y., Lerman, A., \& Lerman, L. O. (2020). Upregulated tumor necrosis factor- $\alpha$ transcriptome and proteome in adipose tissue-derived mesenchymal stem cells from pigs with metabolic syndrome. Cytokine, 130, 155080. https://doi.org/10.1016/j.cyto.2020.155080.

38. Eirin, A., Ferguson, C. M., Zhu, X.-Y., Saadiq, I. M., Tang, H., Lerman, A., \& Lerman, L. O. (2020). Extracellular vesicles released by adipose tissue-derived mesenchymal stromal/stem cells from obese pigs fail to repair the injured kidney. Stem Cell Research, 47, 101877. https://doi.org/10.1016/j.scr.2020.101877.

39. Shen, T., Zheng, Q.-Q., Shen, J., Li, Q.-S., Song, X.-H., Luo, H.B., Hong, C. Y., \& Yao, K. (2018). Effects of adipose-derived Mesenchymal stem cell Exosomes on corneal stromal fibroblast viability and extracellular matrix synthesis. Chinese Medical Journal, 131(6), 704-712. https://doi.org/10.4103/0366-6999. 226889.

40. Safwat, A., Sabry, D., Ragiae, A., Amer, E., Mahmoud, R., \& Shamardan, R. (2018). Adipose mesenchymal stem cellsderived exosomes attenuate retina degeneration of streptozotocin-induced diabetes in rabbits. Journal of Circulating Biomarkers, 7, 1-10. https://doi.org/10.1177/ 1849454418807827.
41. Kalra, H., Simpson, R. J., Ji, H., Aikawa, E., Altevogt, P., Askenase, P., Bond, V. C., Borràs, F. E., Breakefield, X., Budnik, V., Buzas, E., Camussi, G., Clayton, A., Cocucci, E., Falcon-Perez, J. M., Gabrielsson, S., Gho, Y. S., Gupta, D., Harsha, H. C., Hendrix, A., Hill, A. F., Inal, J. M., Jenster, G., Krämer-Albers, E. M., Lim, S. K., Llorente, A., Lötvall, J., Marcilla, A., Mincheva-Nilsson, L., Nazarenko, I., Nieuwland, R., Nolte-'t Hoen, E. N. M., Pandey, A., Patel, T., Piper, M. G., Pluchino, S., Prasad, T. S. K., Rajendran, L., Raposo, G., Record, M., Reid, G. E., Sánchez-Madrid, F., Schiffelers, R. M., Siljander, P., Stensballe, A., Stoorvogel, W., Taylor, D., Thery, C., Valadi, H., van Balkom, B. W. M., Vázquez, J., Vidal, M., Wauben, M. H. M., Yáñez-Mó, M., Zoeller, M., \& Mathivanan, S. (2012). Vesiclepedia: A compendium for extracellular vesicles with continuous community annotation. PLoS Biology, 10(12), e1001450. https://doi.org/10.1371/journal.pbio.1001450.

42. Mathivanan, S., \& Simpson, R. J. (2009). ExoCarta: A compendium of exosomal proteins and RNA. PROTEOMICS, 9(21), 4997-5000. https://doi.org/10.1002/pmic.200900351.

43. Kim, D.-K., Kang, B., Kim, O. Y., Choi, D., Lee, J., Kim, S. R., Go, G., Yoon, Y. J., Kim, J. H., Jang, S. C., Park, K. S., Choi, E. J., Kim, K. P., Desiderio, D. M., Kim, Y. K., Lötvall, J., Hwang, D., \& Gho, Y. S. (2013). EVpedia: An integrated database of highthroughput data for systemic analyses of extracellular vesicles. Journal of Extracellular Vesicles, 2(1), 20384. https://doi.org/10. 3402/jev.v2i0.20384.

44. Apweiler, R., Bairoch, A., Wu, C. H., Barker, W. C., Boeckmann, B., Ferro, S., et al. (2004). UniProt: The universal protein knowledgebase. Nucleic Acids Research, 32(Database issue), D115-D119. https://doi.org/10.1093/nar/gkh131.

45. Sweeney, B. A., Petrov, A. I., Burkov, B., Finn, R. D., Bateman, A., Szymanski, M., et al. (2019). RNAcentral: A hub of information for non-coding RNA sequences. Nucleic Acids Research, 47(D1), D221-D229. https://doi.org/10.1093/nar/gky1034.

46. Griffiths-Jones, S. (2004). The microRNA registry. Nucleic Acids Research, 32, D109-D111. https://doi.org/10.1093/nar/gkh023.

47. Griffiths-Jones, S. (2006). miRBase: microRNA sequences, targets and gene nomenclature. Nucleic Acids Research, 34, D140D144. https://doi.org/10.1093/nar/gkj112.

48. Griffiths-Jones, S., Saini, H. K., van Dongen, S., \& Enright, A. J. (2008). miRBase: Tools for microRNA genomics. Nucleic Acids Research, 36, D154-D158. https://doi.org/10.1093/nar/gkm952.

49. Kozomara, A., \& Griffiths-Jones, S. (2011). miRBase: Integrating microRNA annotation and deep-sequencing data. Nucleic Acids Research, 39, D152-D157. https://doi.org/10.1093/nar/gkq1027.

50. Kozomara, A., \& Griffiths-Jones, S. (2014). miRBase: Annotating high confidence microRNAs using deep sequencing data. Nucleic Acids Research, 42, D68-D73. https://doi.org/10.1093/nar/ gkt1181.

51. Kozomara, A., Birgaoanu, M., \& Griffiths-Jones, S. (2019). miRBase: From microRNA sequences to function. Nucleic Acids Research, 47, D155-D162. https://doi.org/10.1093/nar/ gky1141.

52. Figliolini, F., Ranghino, A., Grange, C., Cedrino, M., Tapparo, M., Cavallari, C., Rossi, A., Togliatto, G., Femminò, S., Gugliuzza, M. V., Camussi, G., \& Brizzi, M. F. (2020). Extracellular vesicles from adipose stem cells prevent muscle damage and inflammation in a mouse model of hind limb ischemia. Arteriosclerosis, Thrombosis, and Vascular Biology, 40(1), 239-254. https://doi.org/10.1161/ATVBAHA.119.313506.

53. Kaur, S., Abu-Shahba, A. G., Paananen, R. O., Hongisto, H., Hiidenmaa, H., \& Skottman, et al. (2018). Small non-coding RNA landscape of extracellular vesicles from human stem cells. Scientific Reports, 8(1), 15503. https://doi.org/10.1038/s41598018-33899-6. 
54. Reza, A. M. M. T., Choi, Y.-J., Yasuda, H., \& Kim, J.-H. (2016). Human adipose mesenchymal stem cell-derived exosomalmiRNAs are critical factors for inducing anti-proliferation signalling to A2780 and SKOV-3 ovarian cancer cells. Scientific Reports, 6(1), 38498. https://doi.org/10.1038/srep38498.

55. Binns, D., Dimmer, E., Huntley, R., Barrell, D., O'Donovan, C., \& Apweiler, R. (2009). QuickGO: A web-based tool for gene ontology searching. Bioinformatics, 25(22), 3045-3046. https:// doi.org/10.1093/bioinformatics/btp536.

56. Ashburner, M., Ball, C. A., Blake, J. A., Botstein, D., Butler, H., Cherry, J. M., Davis, A. P., Dolinski, K., Dwight, S. S., Eppig, J. T., Harris, M. A., Hill, D. P., Issel-Tarver, L., Kasarskis, A., Lewis, S., Matese, J. C., Richardson, J. E., Ringwald, M., Rubin, G. M., \& Sherlock, G. (2000). Gene ontology: Tool for the unification of biology. Nature Genetics, 25(1), 25-29. https:// doi.org/10.1038/75556.

57. Wang, X., Shah, F. A., Vazirisani, F., Johansson, A., Palmquist, A., Omar, O., Ekström, K., \& Thomsen, P. (2020). Exosomes influence the behavior of human mesenchymal stem cells on titanium surfaces. Biomaterials, 230, 119571. https://doi.org/10. 1016/j.biomaterials.2019.119571.

58. Bari, E., Ferrarotti, I., Di Silvestre, D., Grisoli, P., Barzon, V., Balderacchi, A., et al. (2019). Adipose Mesenchymal extracellular vesicles as Alpha-1-antitrypsin physiological delivery Systems for Lung Regeneration. Cells, 8(9), 965. https://doi.org/10.3390/ cells8090965.

59. Mayo, J. S., Kurata, W. E., O’Connor, K. M., \& Pierce, L. M. (2019). Oxidative stress alters Angiogenic and antimicrobial content of extracellular vesicles and improves flap survival. Plastic and Reconstructive Surgery - Global Open, 7(12), e2588. https:// doi.org/10.1097/GOX.0000000000002588.

60. Lopatina, T., Bruno, S., Tetta, C., Kalinina, N., Porta, M., \& Camussi, G. (2014). Platelet-derived growth factor regulates the secretion of extracellular vesicles by adipose mesenchymal stem cells and enhances their angiogenic potential. Cell Communication and Signaling, 12(1), 26. https://doi.org/10. 1186/1478-811X-12-26.

61. Han, Y., Ren, J., Bai, Y., Pei, X., \& Han, Y. (2019). Exosomes from hypoxia-treated human adipose-derived mesenchymal stem cells enhance angiogenesis through VEGF/VEGF-R. The International Journal of Biochemistry \& Cell Biology, 109, 5968. https://doi.org/10.1016/j.biocel.2019.01.017.

62. Pu, C.-M., Liu, C.-W., Liang, C.-J., Yen, Y.-H., Chen, S.-H., Jiang-Shieh, Y.-F., Chien, C. L., Chen, Y. C., \& Chen, Y. L. (2017). Adipose-derived stem cells protect skin flaps against ischemia/reperfusion injury via IL-6 expression. Journal of Investigative Dermatology, 137(6), 1353-1362. https://doi.org/ 10.1016/j.jid.2016.12.030.

63. Katsuda, T., Tsuchiya, R., Kosaka, N., Yoshioka, Y., Takagaki, K., Oki, K., Takeshita, F., Sakai, Y., Kuroda, M., \& Ochiya, T. (2013). Human adipose tissue-derived mesenchymal stem cells secrete functional neprilysin-bound exosomes. Scientific Reports, 3(1), 1197. https://doi.org/10.1038/srep01197.

64. Lopatina, T., Mazzeo, A., Bruno, S., Tetta, C., Kalinina, N., Romagnoli, R., et al. (2014). The Angiogenic potential of adipose Mesenchymal stem cell-derived extracellular vesicles is modulated by basic fibroblast growth factor. Journal of Stem Cell Research \& Therapy, 4(10), 245. https://doi.org/10.4172/21577633.1000245.

65. Lopatina, T., Favaro, E., Grange, C., Cedrino, M., Ranghino, A., Occhipinti, S., Fallo, S., Buffolo, F., Gaykalova, D. A., Zanone, M. M., Romagnoli, R., \& Camussi, G. (2018). PDGF enhances the protective effect of adipose stem cell-derived extracellular vesicles in a model of acute hindlimb ischemia. Scientific Reports, 8(1), 17458. https://doi.org/10.1038/s41598-018-36143-3.
66. Woo, C. H., Kim, H. K., Jung, G. Y., Jung, Y. J., Lee, K. S., Yun, Y. E., Han, J., Lee, J., Kim, W. S., Choi, J. S., Yang, S., Park, J. H., Jo, D. G., \& Cho, Y. W. (2020). Small extracellular vesicles from human adipose-derived stem cells attenuate cartilage degeneration. Journal of Extracellular Vesicles, 9(1), 1735249. https:// doi.org/10.1080/20013078.2020.1735249.

67. Chen, L., Mou, S., Li, F., Zeng, Y., Sun, Y., Horch, R. E., Wei, W., Wang, Z., \& Sun, J. (2019). Self-assembled human adiposederived stem cell-derived extracellular vesicle-functionalized biotin-doped Polypyrrole titanium with long-term stability and potential Osteoinductive ability. ACS Applied Materials \& Interfaces, 11(49), 46183-46196. https://doi.org/10.1021/acsami.9b17015.

68. da Ferreira, A., F., da Cunha, P., S., Carregal, V. M., de Silva, P., C., da, M. M. C., de, K.-L. M., et al. (2017). Extracellular vesicles from adipose-derived Mesenchymal stem/stromal cells accelerate migration and activate AKT pathway in human keratinocytes and fibroblasts independently of miR-205 activity. Stem Cells International, 2017, 9841035-9841014. https://doi.org/10.1155/ 2017/9841035.

69. Ragni, E., Perucca Orfei, C., De Luca, P., Colombini, A., Viganò, M., Lugano, G., et al. (2019). Identification of miRNA reference genes in extracellular vesicles from adipose derived Mesenchymal stem cells for studying osteoarthritis. International Journal of Molecular Sciences, 20(5), 1108. https://doi.org/10.3390/ ijms20051108.

70. Ragni, E., Perucca Orfei, C., De Luca, P., Viganò, M., Colombini, A., Lugano, G., et al. (2019). miR-22-5p and miR-29a-5p are reliable reference genes for analyzing extracellular vesicleassociated miRNAs in adipose-derived Mesenchymal stem cells and are stable under inflammatory priming mimicking osteoarthritis condition. Stem Cell Reviews and Reports, 15(5), 743-754. https://doi.org/10.1007/s12015-019-09899-y.

71. Domenis, R., Cifù, A., Quaglia, S., Pistis, C., Moretti, M., Vicario, A., Parodi, P. C., Fabris, M., Niazi, K. R., Soon-Shiong, P., \& Curcio, F. (2018). Pro inflammatory stimuli enhance the immunosuppressive functions of adipose mesenchymal stem cells-derived exosomes. Scientific Reports, 8(1), 13325. https://doi.org/10. 1038/s41598-018-31707-9.

72. Huang, B., Huang, L.-F., Zhao, L., Zeng, Z., Wang, X., Cao, D., Yang, L., Ye, Z., Chen, X., Liu, B., He, T. C., \& Wang, X. (2020). Microvesicles (MIVs) secreted from adipose-derived stem cells (ADSCs) contain multiple microRNAs and promote the migration and invasion of endothelial cells. Genes \& Diseases, 7(2), 225234. https://doi.org/10.1016/j.gendis.2019.04.005.

73. Wang, K., Jiang, Z., Webster, K. A., Chen, J., Hu, H., Zhou, Y., Zhao, J., Wang, L., Wang, Y., Zhong, Z., Ni, C., Li, Q., Xiang, C., Zhang, L., Wu, R., Zhu, W., Yu, H., Hu, X., \& Wang, J.'. (2017). Enhanced Cardioprotection by human endometrium Mesenchymal stem cells driven by Exosomal MicroRNA-21. Stem Cells Translational Medicine, 6(1), 209-222. https://doi. org/10.5966/sctm.2015-0386.

74. Nakamura, Y., Kita, S., Tanaka, Y., Fukuda, S., Obata, Y., Okita, T., Nishida, H., Takahashi, Y., Kawachi, Y., Tsugawa-Shimizu, Y., Fujishima, Y., Nishizawa, H., Takakura, Y., Miyagawa, S., Sawa, Y., Maeda, N., \& Shimomura, I. (2020). Adiponectin stimulates exosome release to enhance Mesenchymal stem-cell-driven therapy of heart failure in mice. Molecular Therapy, 28, 1-17. https://doi.org/10.1016/j.ymthe.2020.06.026.

75. Li, W., Liu, Y., Zhang, P., Tang, Y., Zhou, M., Jiang, W., Zhang, X., Wu, G., \& Zhou, Y. (2018). Tissue-engineered bone immobilized with human adipose stem cells-derived Exosomes promotes bone regeneration. ACS Applied Materials \& Interfaces, 10(6), 5240-5254. https://doi.org/10.1021/acsami. $7 \mathrm{~b} 17620$.

76. Gandolfi, M. G., Gardin, C., Zamparini, F., Ferroni, L., Esposti, M. D., Parchi, G., Ercan, B., Manzoli, L., Fava, F., Fabbri, P., 
Prati, C., \& Zavan, B. (2020). Mineral-doped poly(L-lactide) acid scaffolds enriched with Exosomes improve Osteogenic commitment of human adipose-derived Mesenchymal stem cells. Nanomaterials, 10(3), 432. https://doi.org/10.3390/ nano10030432.

77. Chen, S., Tang, Y., Liu, Y., Zhang, P., Lv, L., Zhang, X., Jia, L., \& Zhou, Y. (2019). Exosomes derived from miR-375overexpressing human adipose mesenchymal stem cells promote bone regeneration. Cell Proliferation, 52(5), e12669. https://doi. org/10.1111/cpr.12669.

78. Tofiño-Vian, M., Guillén, M. I., Pérez del Caz, M. D., Castejón, M. A., \& Alcaraz, M. J. (2017). Extracellular vesicles from adipose-derived Mesenchymal stem cells Downregulate senescence features in osteoarthritic osteoblasts. Oxidative Medicine and Cellular Longevity, 2017, 7197598-7197512. https://doi. org/10.1155/2017/7197598.

79. Xu, H., Wang, Z., Liu, L., Zhang, B., \& Li, B. (2020). Exosomes derived from adipose tissue, bone marrow, and umbilical cord blood for cardioprotection after myocardial infarction. Journal of Cellular Biochemistry, 121(3), 2089-2102. https://doi.org/10. $1002 /$ jcb. 27399.

80. Almeria, C., Weiss, R., Roy, M., Tripisciano, C., Kasper, C., Weber, V., \& Egger, D. (2019). Hypoxia conditioned Mesenchymal stem cell-derived extracellular vesicles induce increased vascular tube formation in vitro. Frontiers in Bioengineering and Biotechnology, 7, 292. https://doi.org/10. 3389/fbioe.2019.00292.

81. Liu, R., Shen, H., Ma, J., Sun, L., \& Wei, M. (2016). Extracellular vesicles derived from adipose Mesenchymal stem cells regulate the phenotype of smooth muscle cells to limit intimal hyperplasia. Cardiovascular Drugs and Therapy, 30(2), 111-118. https://doi. org/10.1007/s10557-015-6630-5.

82. Lindoso, R. S., Lopes, J. A., Binato, R., Abdelhay, E., Takiya, C. M., de Miranda, K. R., et al. (2020). Adipose Mesenchymal cellsderived EVs alleviate DOCA-salt-induced hypertension by promoting cardio-renal protection. Molecular Therapy - Methods \& Clinical Development, 16, 63-77. https://doi.org/10.1016/j.omtm. 2019.11.002.

83. Gao, F., Zuo, B., Wang, Y., Li, S., Yang, J., \& Sun, D. (2020). Protective function of exosomes from adipose tissue-derived mesenchymal stem cells in acute kidney injury through SIRT1 pathway. Life Sciences, 255, 117719. https://doi.org/10.1016/j.lfs. 2020.117719.

84. Ma, T., Fu, B., Yang, X., Xiao, Y., \& Pan, M. (2019). Adipose mesenchymal stem cell-derived exosomes promote cell proliferation, migration, and inhibit cell apoptosis via $\mathrm{Wnt} / \beta$-catenin signaling in cutaneous wound healing. Journal of Cellular Biochemistry, 120(6), 10847-10854. https://doi.org/10.1002/jcb. 28376.

85. Wang, L., Hu, L., Zhou, X., Xiong, Z., Zhang, C., Shehada, H. M. A., Hu, B., Song, J., \& Chen, L. (2017). Exosomes secreted by human adipose mesenchymal stem cells promote scarless cutaneous repair by regulating extracellular matrix remodelling. Scientific Reports, 7(1), 13321. https://doi.org/10.1038/s41598017-12919-X.

86. Bai, Y., Han, Y., Yan, X., Ren, J., Zeng, Q., Li, X., Pei, X. T., \& Han, Y. (2018). Adipose mesenchymal stem cell-derived exosomes stimulated by hydrogen peroxide enhanced skin flap recovery in ischemia-reperfusion injury. Biochemical and Biophysical Research Communications, 500(2), 310-317. https://doi.org/10.1016/j.bbrc.2018.04.065.

87. Serejo, T. R. T., Silva-Carvalho, A. É., de Braga, L. D., C. F., de Neves, F., A. R., Pereira, R. W., de Carvalho, J. L., \& SaldanhaAraujo, F. (2019). Assessment of the immunosuppressive potential of INF- $\gamma$ licensed adipose Mesenchymal stem cells, their
Secretome and extracellular vesicles. Cells, 8(1), 22. https://doi. org/10.3390/cells 8010022 .

88. Yu, C., Chen, P., Xu, J., Liu, Y., Li, H., Wang, L., \& Di, G. (2020). hADSCs derived extracellular vesicles inhibit NLRP3inflammasome activation and dry eye. Scientific Reports, 10(1), 14521. https://doi.org/10.1038/s41598-020-71337-8.

89. Rajashekhar, G., Ramadan, A., Abburi, C., Callaghan, B., Traktuev, D. O., Evans-Molina, C., Maturi, R., Harris, A., Kern, T. S., \& March, K. L. (2014). Regenerative therapeutic potential of adipose stromal cells in early stage diabetic retinopathy. PLoS One, 9(1), e84671. https://doi.org/10.1371/journal.pone.0084671.

90. Sugitani, S., Tsuruma, K., Ohno, Y., Kuse, Y., Yamauchi, M., Egashira, Y., Yoshimura, S., Shimazawa, M., Iwama, T., \& Hara, H. (2013). The potential neuroprotective effect of human adipose stem cells conditioned medium against light-induced retinal damage. Experimental Eye Research, 116, 254-264. https:// doi.org/10.1016/j.exer.2013.09.013.

91. Elshaer, S. L., Evans, W., Pentecost, M., Lenin, R., Periasamy, R., Jha, K. A., Alli, S., Gentry, J., Thomas, S. M., Sohl, N., \& Gangaraju, R. (2018). Adipose stem cells and their paracrine factors are therapeutic for early retinal complications of diabetes in the Ins2Akita mouse. Stem Cell Research \& Therapy, 9(1), 322. https://doi.org/10.1186/s13287-018-1059-y.

92. Jha, K., Pentecost, M., Lenin, R., Klaic, L., Elshaer, S., Gentry, J., Russell, J., Beland, A., Reiner, A., Jotterand, V., Sohl, N., \& Gangaraju, R. (2018). Concentrated conditioned media from adipose tissue derived Mesenchymal stem cells mitigates visual deficits and retinal inflammation following mild traumatic brain injury. International Journal of Molecular Sciences, 19(7), 2016. https://doi.org/10.3390/ijms19072016.

93. Zhang, M., Zhang, F., Sun, J., Sun, Y., Xu, L., Zhang, D., Wang, Z., \& He, W. (2017). The condition medium of mesenchymal stem cells promotes proliferation, adhesion and neuronal differentiation of retinal progenitor cells. Neuroscience Letters, 657, 62-68. https://doi.org/10.1016/j.neulet.2017.07.053.

94. Haddad-Mashadrizeh, A., Bahrami, A. R., Matin, M. M., Edalatmanesh, M. A., Zomorodipour, A., Gardaneh, M., Farshchian, M., \& Momeni-Moghaddam, M. (2013). Human adipose-derived mesenchymal stem cells can survive and integrate into the adult rat eye following xenotransplantation. Xenotransplantation, 20(3), 165-176. https://doi.org/10.1111/ xen. 12033.

95. Galindo, S., Herreras, J. M., López-Paniagua, M., Rey, E., de la Mata, A., Plata-Cordero, M., Calonge, M., \& Nieto-Miguel, T. (2017). Therapeutic effect of human adipose tissue-derived Mesenchymal stem cells in experimental corneal failure due to Limbal stem cell niche damage. Stem Cells, 35(10), 2160-2174. https://doi.org/10.1002/stem.2672.

96. Bandeira, F., Goh, T.-W., Setiawan, M., Yam, G. H.-F., \& Mehta, J. S. (2020). Cellular therapy of corneal epithelial defect by adipose mesenchymal stem cell-derived epithelial progenitors. Stem Cell Research \& Therapy, 11(1), 14. https://doi.org/10.1186/ s13287-019-1533-1.

97. Nieto-Miguel, T., Galindo, S., Reinoso, R., Corell, A., Martino, M., Pérez-Simón, J. A., \& Calonge, M. (2013). In vitro simulation of corneal epithelium microenvironment induces a corneal epithelial-like cell phenotype from human adipose tissue Mesenchymal stem cells. Current Eye Research, 38(9), 933944. https://doi.org/10.3109/02713683.2013.802809.

98. Singh, A. K., Srivastava, G. K., García-Gutiérrez, M. T., \& Pastor, J. C. (2013). Adipose derived mesenchymal stem cells partially rescue mitomycin C treated ARPE19 cells from death in coculture condition. Histology and Histopathology, 28(12), 15771583. https://doi.org/10.14670/HH-28.1577.

99. Alonso-Alonso, M. L., Srivastava, G. K., Usategui-Martín, R., García-Gutierrez, M. T., Pastor, J. C., \& Fernandez-Bueno, I. 
(2020). Mesenchymal stem cell Secretome enhancement by Nicotinamide and vasoactive intestinal peptide: A new therapeutic approach for retinal degenerative diseases. Stem Cells International, 2020, 1-14. https://doi.org/10.1155/2020/9463548.

100. Mead, B., Chamling, X., Zack, D. J., Ahmed, Z., \& Tomarev, S. (2020). TNF $\alpha$-mediated priming of Mesenchymal stem cells enhances their Neuroprotective effect on retinal ganglion cells. Investigative Opthalmology \& Visual Science, 61(2), 6. https:// doi.org/10.1167/iovs.61.2.6.

101. Mead, B., \& Tomarev, S. (2017). Bone marrow-derived Mesenchymal stem cells-derived Exosomes promote survival of retinal ganglion cells through miRNA-dependent mechanisms. Stem Cells Translational Medicine, 6(4), 1273-1285. https://doi. org/10.1002/sctm.16-0428.

102. Mead, B., Amaral, J., \& Tomarev, S. (2018). Mesenchymal stem cell-derived small extracellular vesicles promote Neuroprotection in rodent models of glaucoma. Investigative Opthalmology \& Visual Science, 59(2), 702-714. https://doi.org/10.1167/iovs.17-22855.

103. Mead, B., Ahmed, Z., \& Tomarev, S. (2018). Mesenchymal stem cell-derived small extracellular vesicles promote Neuroprotection in a genetic DBA/2J mouse model of glaucoma. Investigative Opthalmology \& Visual Science, 59(13), 5473-5480. https://doi. org/10.1167/iovs.18-25310.

104. Wang, J.-D., An, Y., Zhang, J.-S., Wan, X.-H., Jonas, J. B., Xu, L., \& Zhang, W. (2017). Human bone marrow mesenchymal stem cells for retinal vascular injury. Acta Ophthalmologica, 95(6), e453-e461. https://doi.org/10.1111/aos.13154.

105. Tzameret, A., Sher, I., Belkin, M., Treves, A. J., Meir, A., Nagler, A., Levkovitch-Verbin, H., Rotenstreich, Y., \& Solomon, A. S. (2015). Epiretinal transplantation of human bone marrow mesenchymal stem cells rescues retinal and vision function in a rat model of retinal degeneration. Stem Cell Research, 15(2), 387-394. https://doi.org/10.1016/j.scr.2015.08.007.

106. Tzameret, A., Sher, I., Belkin, M., Treves, A. J., Meir, A., Nagler, A., Levkovitch-Verbin, H., Barshack, I., Rosner, M., \& Rotenstreich, Y. (2014). Transplantation of human bone marrow mesenchymal stem cells as a thin subretinal layer ameliorates retinal degeneration in a rat model of retinal dystrophy. Experimental Eye Research, 118, 135144. https://doi.org/10.1016/j.exer.2013.10.023.

107. Jeong, W.-Y., Kim, J.-H., \& Kim, C.-W. (2018). Co-culture of human bone marrow mesenchymal stem cells and macrophages attenuates lipopolysaccharide-induced inflammation in human corneal epithelial cells. Bioscience, Biotechnology, and Biochemistry, 82(5), 800-809. https://doi.org/10.1080/09168451. 2018.1438167.

108. Usategui-Martín, R., Puertas-Neyra, K., García-Gutiérrez, M.-T., Fuentes, M., Pastor, J. C., \& Fernandez-Bueno, I. (2020). Human Mesenchymal stem cell Secretome exhibits a Neuroprotective effect over in vitro retinal photoreceptor degeneration. Molecular Therapy - Methods \& Clinical Development, 17, 1155-1166. https://doi.org/10.1016/j.omtm.2020.05.003.

109. Labrador-Velandia, S., Alonso-Alonso, M. L., Di Lauro, S., García-Gutierrez, M. T., Srivastava, G. K., Pastor, J. C., \& Fernandez-Bueno, I. (2019). Mesenchymal stem cells provide paracrine neuroprotective resources that delay degeneration of co-cultured organotypic neuroretinal cultures. Experimental Eye Research, 185, 107671. https://doi.org/10.1016/j.exer.2019.05. 011.
110. Labrador Velandia, S., Di Lauro, S., Alonso-Alonso, M. L., Tabera Bartolomé, S., Srivastava, G. K., Pastor, J. C., \& Fernandez-Bueno, I. (2018). Biocompatibility of intravitreal injection of human mesenchymal stem cells in immunocompetent rabbits. Graefe's Archive for Clinical and Experimental Ophthalmology, 256(1), 125-134. https://doi.org/10.1007/ s00417-017-3842-3.

111. Gu, X., Yu, X., Zhao, C., Duan, P., Zhao, T., Liu, Y., Li, S., Yang, Z., Li, Y., Qian, C., Yin, Z., \& Wang, Y. (2018). Efficacy and safety of autologous bone marrow Mesenchymal stem cell transplantation in patients with diabetic retinopathy. Cellular Physiology and Biochemistry, 49(1), 40-52. https://doi.org/10. $1159 / 000492838$.

112. Nakahara, M., Okumura, N., Kay, E. P., Hagiya, M., Imagawa, K., Hosoda, Y., Kinoshita, S., \& Koizumi, N. (2013). Corneal endothelial expansion promoted by human bone marrow Mesenchymal stem cell-derived conditioned medium. PLoS One, 8(7), e69009. https://doi.org/10.1371/journal.pone.0069009.

113. Fernandes-Cunha, G. M., Na, K., Putra, I., Lee, H. J., Hull, S., Cheng, Y., et al. (2019). Corneal wound healing effects of Mesenchymal stem cell Secretome delivered within a viscoelastic gel carrier. Stem Cells Translational Medicine, 8(5), 478-489. https://doi.org/10.1002/sctm.18-0178.

114. Calonge, M., Pérez, I., Galindo, S., Nieto-Miguel, T., LópezPaniagua, M., Fernández, I., Alberca, M., García-Sancho, J., Sánchez, A., \& Herreras, J. M. (2019). A proof-of-concept clinical trial using mesenchymal stem cells for the treatment of corneal epithelial stem cell deficiency. Translational Research, 206, 1840. https://doi.org/10.1016/j.trsl.2018.11.003.

115. Weiss, J., Levy, S., \& Benes, S. (2016). Stem cell ophthalmology treatment study (SCOTS): Bone marrow-derived stem cells in the treatment of Leber's hereditary optic neuropathy. Neural Regeneration Research, 11(10), 1685-1694. https://doi.org/10. 4103/1673-5374.193251.

116. Weiss, J., Benes, S., \& Levy, S. (2016). Stem Cell Ophthalmology Treatment Study (SCOTS): improvement in serpiginous choroidopathy following autologous bone marrow derived stem cell treatment. Neural Regeneration Research, 11(9), 1512-1516. https://doi.org/10.4103/1673-5374.191229.

117. Weiss, J. N., \& Levy, S. (2019). Stem cell ophthalmology treatment study (SCOTS): Bone marrow derived stem cells in the treatment of dominant optic atrophy. Stem Cell Investigation, 6, 41-41. https://doi.org/10.21037/sci.2019.11.01.

118. Zhang, K., Dong, C., Chen, M., Yang, T., Wang, X., Gao, Y., Wang, L., Wen, Y., Chen, G., Wang, X., Yu, X., Zhang, Y., Wang, P., Shang, M., Han, K., \& Zhou, Y. (2020). Extracellular vesicle-mediated delivery of miR-101 inhibits lung metastasis in osteosarcoma. Theranostics, 10(1), 411-425. https://doi.org/10. 7150/thno.33482.

119. Baldari, S., Di Rocco, G., Magenta, A., Picozza, M., \& Toietta, G. (2019). Extracellular vesicles-encapsulated MicroRNA-125b produced in genetically modified Mesenchymal stromal cells inhibits hepatocellular carcinoma cell proliferation. Cells, 8(12), 1560. https://doi.org/10.3390/cells8121560.

Publisher's Note Springer Nature remains neutral with regard to jurisdictional claims in published maps and institutional affiliations. 